\title{
Cardiac Applications of Hyperpolarised Magnetic Resonance
}

\author{
Kerstin N. Timm ${ }^{1,}$, Jack J. Miller ${ }^{1,2,3}$, John A. Henry ${ }^{1}$ and Damian J. Tyler ${ }^{1,2}$ \\ ${ }^{1}$ Department of Physiology, Anatomy and Genetics, University of Oxford (UK) \\ ${ }^{2}$ Oxford Centre for Clinical Magnetic Resonance Research, John Radcliffe Hospital, Oxford (UK) \\ ${ }^{3}$ Clarendon Laboratory, Department of Physics, University of Oxford (UK)
}

*corresponding author

Department of Physiology Anatomy and Genetics,

University of Oxford, Sherrington Building,

Parks Road, Oxford

OX1 3PT

Email address: kerstin.timm@dpag.ox.ac.uk

Telephone number: +44 (0)1865 2725690

Email addresses of co-authors:

jack.miller@physics.org

john.henry@some.ox.ac.uk

damian.tyler@dpag.ox.ac.uk

Number of pages: 63

Number of Tables: 2

Number of Figures: 9

Keywords: hyperpolarised; ${ }^{13} \mathrm{C}$; DNP; cardiac; spectroscopy 


\begin{abstract}
Cardiovascular disease is the leading cause of death world-wide. It is increasingly recognised that cardiac pathologies show, or may even be caused by, changes in metabolism, leading to impaired cardiac energetics. The heart turns over 15 times its own weight in ATP every day and thus relies heavily on the availability of substrates and on efficient oxidation to generate this ATP. A number of old and emerging drugs that target different aspects of metabolism are showing promising results with regard to improved cardiac outcomes in patients. A non-invasive imaging technique that could assess the role of different aspects of metabolism in heart disease, as well as measure changes in cardiac energetics due to treatment, would be valuable in the routine clinical care of cardiac patients. Hyperpolarised magnetic resonance spectroscopy and imaging have revolutionised metabolic imaging, allowing real-time metabolic flux assessment in vivo for the first time. In this review we summarise metabolism in the healthy and diseased heart, give an introduction to the hyperpolarisation technique, 'dynamic nuclear polarisation' (DNP), and review the preclinical studies that have thus far explored healthy cardiac metabolism and different models of human heart disease. We furthermore show what advances have been made to translate this technique into the clinic, what technical challenges still remain and what unmet clinical needs and unexplored metabolic substrates still need to be assessed by researchers in this exciting and fast-moving field.
\end{abstract}




\section{Table of contents}

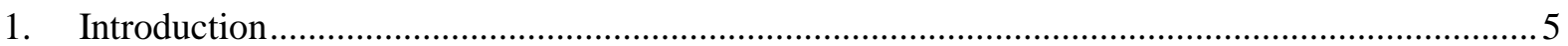

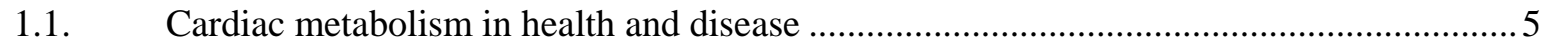

$1.2 \quad$ Clinical imaging modalities to assess cardiac metabolism ............................................... 7

1.3 Hyperpolarised ${ }^{13} \mathrm{C}$ magnetic resonance spectroscopy by dynamic nuclear polarisation ...9

2. Cardiac applications of hyperpolarised magnetic resonance .......................................................11

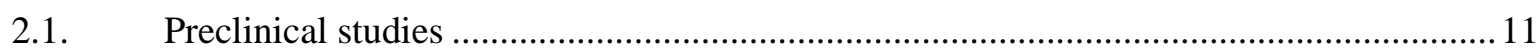

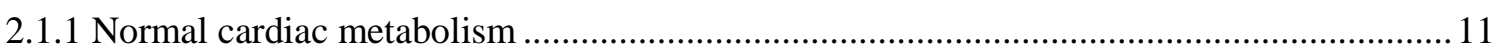

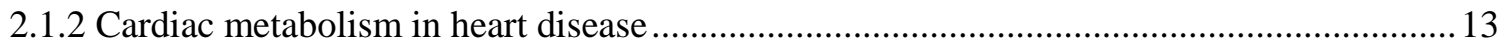

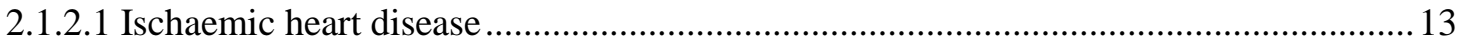

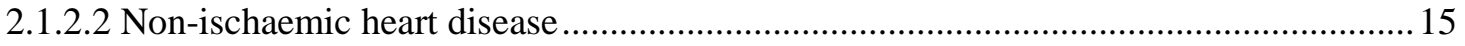

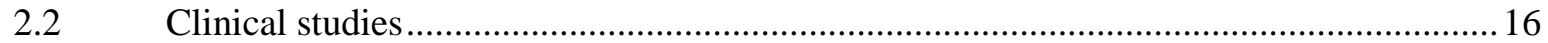

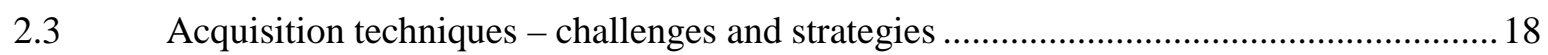

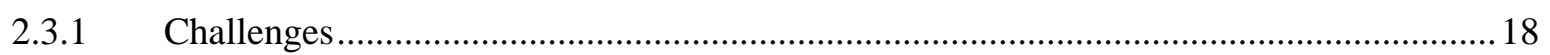

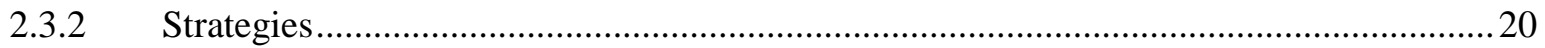

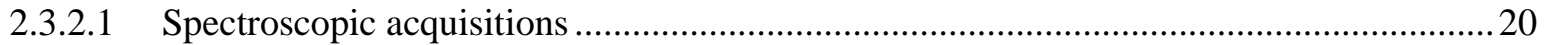

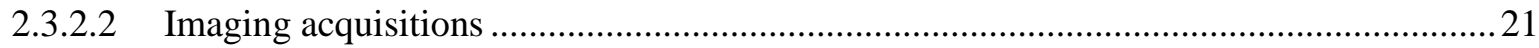

3. Future applications of cardiac hyperpolarised magnetic resonance spectroscopy ….....................25

3.1 Hyperpolarised substrates for potential use in cardiac applications ...................................25

3.2 Improving and extending substrates for hyperpolarised MRI..........................................26

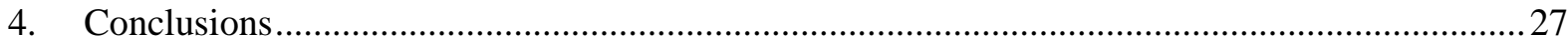

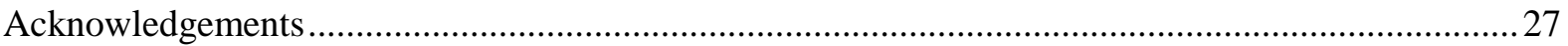

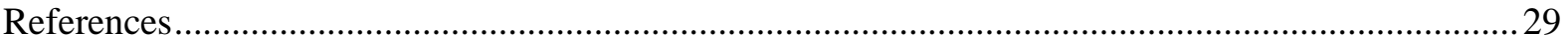

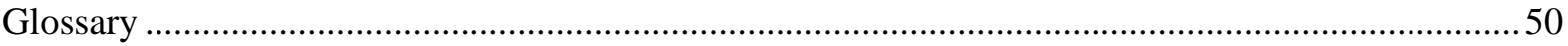

\section{Tables}

1. List of preclinical models of heart disease explored by hyperpolarised magnetic resonance

2. Pulse sequences for hyperpolarised magnetic resonance imaging of metabolism and their properties

\section{Figures}

1. Cardiac metabolism

2. Measuring TCA cycle flux with hyperpolarised $\left[1-{ }^{13} \mathrm{C}\right]$ - and $\left[2-{ }^{13} \mathrm{C}\right]$ pyruvate

3. Hyperpolarised $\left[1-{ }^{13} \mathrm{C}\right]$ pyruvate MR spectroscopy in healthy rat hearts

4. Hyperpolarised $\left[1-{ }^{13} \mathrm{C}\right]$ pyruvate imaging in a healthy pig heart after GIK infusion 
5. Intracellular $\mathrm{pH}$ imaging with hyperpolarised $\left[1-{ }^{13} \mathrm{C}\right]$ pyruvate in rat hearts at rest and under dobutamine stress

6. Imaging hyperpolarised $\left[1-{ }^{13} \mathrm{C}\right]$ pyruvate metabolism in a rat model of myocardial infarction

7. Imaging tissue necrosis with hyperpolarised $\left[1,4-{ }^{13} \mathrm{C}_{2}\right]$ fumarate in a rat model of myocardial infarction

8. Hyperpolarised $\left[2-{ }^{13} \mathrm{C}\right]$ pyruvate MR spectroscopy in a pig model of dilated cardiomyopathy

9. First in-man hyperpolarised $\left[1-{ }^{13} \mathrm{C}\right]$ pyruvate MR spectroscopy of the heart 


\section{Introduction}

\subsection{Cardiac metabolism in health and disease}

The heart supplies the rest of the body with oxygenated blood through its constant contractions. In order to keep beating, the heart relies on a continuous supply of energy in the form of the high energy phosphate, ATP. This ATP is generated in metabolic pathways that have evolved to be energetically efficient [1] and as such are tightly controlled, highly regulated and finely-tuned to meet the heart's constant demand for energy. Impressively, the heart turns over 15 times its own weight in ATP every day [2]. The healthy heart generates $95 \%$ of its ATP through oxidative metabolism in the mitochondria. The foetal heart relies heavily on carbohydrates as a fuel for this oxidative metabolism [3], while the adult heart preferentially uses fatty acids [4]. In fact, the healthy adult heart generates approximately $70 \%$ of its ATP from the oxidation of fatty acids, while the remainder is produced from glucose, lactate, ketone bodies and amino acids [5].

Fatty acid and glucose oxidation are reciprocally regulated by the Randle cycle [6], [7] so that the healthy heart can modulate its fuel choice based on substrate supply and energetic demand. This ensures constant ATP production regardless of the type of fuel provided by the blood stream. The key regulatory enzyme of the Randle cycle is the pyruvate dehydrogenase (PDH) complex, the gatekeeper enzyme for glucose oxidation in the mitochondria. Fatty acids produce more ATP per mole than glucose, but due to their highly reduced structure require more oxygen for complete oxidation in the mitochondria compared to glucose. Blood glucose levels increase after a meal and subsequently glucose is taken up into tissues. The predominant glucose transporter (GLUT) in the adult heart is GLUT4 [8], which is stored in intracellular vesicles and incorporated into the plasma membrane in response to feeding (insulin release) and exercise (catecholamine mediated). Around 40\% of glucose transporters are GLUT1, whose expression is increased in hypoxia [9] and in response to prolonged fasting [10]. Free fatty acid (FFA) levels in the blood are increased after fasting, during stress [11] and in pathological conditions such as diabetes [12]. FFAs enter cardiomyocytes through fatty acid transporters (FAT or CD36) in the plasma membrane or via a 'flip-flop' mechanism [13]. Fatty acids are then transported into the mitochondria for $\beta$-oxidation via the carnitine shuttle [14]. The acetylCoA derived from both pyruvate and fatty acid oxidation enters the tricarboxylic acid (TCA) cycle where its oxidation leads to the reduction of electron carriers, $\mathrm{NAD}^{+}$and FAD. Those electron carriers donate their electrons to the electron transport chain which subsequently allows a proton gradient to be established across the inner mitochondrial membrane. Flow-back of protons into the mitochondrial matrix powers ATP synthase. An overview of cardiac metabolism and ATP generation and the role of ATP and $\mathrm{Ca}^{2+}$ in myofibril contraction are outlined in Figure 1.

Cardiovascular disease is a leading cause of death globally, according to the World Health Organisation, accounting for 31\% of all deaths world-wide in 2015 [15]. Metabolic flexibility is vital 
for cardiac health and is impaired in many different cardiac pathologies. For example, the diabetic heart relies even more heavily on fatty acid oxidation [16] while hypoxic, hypertrophic and failing hearts show a more "foetal" metabolism and preferentially oxidise glucose [17]. This shows that the balance of fatty acid and glucose oxidation in the heart has to be fine-tuned according to demand, and too much or too little fatty acid oxidation can be detrimental [18]. As such, impaired cardiac energetics have been suggested as the driving force in the aetiology [19], pathophysiology [20] and progression [21] of heart failure. Therefore, therapeutically targeting metabolism has gained renewed scientific and clinical interest in recent years [22].

Metabolic targets are increasingly being suggested for the treatment of heart failure [23], as patients with heart failure show altered cardiac substrate utilisation [24]. The failing heart switches from predominantly fatty acid oxidation to increased levels of glucose oxidation, and with disease progression glucose utilisation becomes inefficient with uncoupling of glycolytic metabolism from glucose oxidation [20]. Glucose oxidation requires $11-13 \%$ less $\mathrm{O}_{2}$ for ATP generation than fatty acid oxidation [25], suggesting that increasing glucose oxidation and inhibiting fatty acid oxidation may improve cardiac function in heart failure. To this end a number of agents have been trialled in heart failure [26]. The most promising drugs to target impaired energetics so far have been perhexiline, a carnitine-palmitoyltransferase (CPT-1) inhibitor [27], trimetazidine, an inhibitor of $\beta$-oxidation [28] and dichloroacetate (DCA), an inhibitor of PDH kinase which leads to activation of PDH and improved glucose oxidation [29].

The heart can oxidise not only fatty acids and glucose, but also other metabolic fuels such as branched-chain amino acids (BCAA) and ketone bodies. BCAA supplementation has been shown to extend life-span in mice and to promote mitochondrial biogenesis in cardiac muscle [30]. However, BCAA accumulation in the heart leads to impaired glucose oxidation through inhibition of PDH and sensitises the myocardium to ischaemia-reperfusion injury [31]. Defective BCAA catabolism is also thought to play a role in the development of heart failure [32]. It therefore seems that BCAAs, just like fatty acids, can be either a friend or foe of the heart and targeting their metabolism may be one treatment option for heart failure patients. BCAA supplementation in rats and patients with heart failure and cardiac cachexia has been shown to lead to improved cardiac function and body composition [33], [34] and a clinical trial is now under way to assess cardiopulmonary function and metabolism in cardiac rehabilitation in comparison to classical rehabilitation methods [35].

In recent years, ketone bodies have also gained interest as natural substrates to fuel and support cardiac function in disease states [36]. Ketones are produced from acetyl-CoA by the liver in times of starvation or in untreated diabetes and they act as alternative metabolic fuels for the brain and other organs, such as the heart, if glucose is limiting. Long-chain fatty acid-derived acetyl-CoA is the primary substrate for ketogenesis. Ketosis can also be achieved without starvation through 
carbohydrate-restricted and fat-rich diets as well as through ingestion of ketone salts or ester [37]. Recently ketone-esters have been used to show increased athletic performance in elite athletes [38] and the same ketone-esters have shown improved cardiac energetics in isolated perfused rat hearts [39].

In order to allow for efficient oxidative metabolism in the mitochondria, TCA cycle intermediates need to be replenished, and the process by which net carbon is added to the TCA cycle is called anaplerosis. Anaplerotic reactions include carboxylation of pyruvate by pyruvate carboxylase (PC), which forms oxaloacetate, oxidative decarboxylation of malate to pyruvate by malic enzyme, transamination reactions of the amino acids, aspartate and glutamate, to their ketoacid counterparts, oxaloacetate and $\alpha$-ketoglutarate, as well as formation of fumarate through the purine nucleotide cycle and succinyl-CoA formation from odd-chain fatty acids and BCAAs. Imbalances in cardiac anaplerotic reactions are thought to play a role in a range of cardiac pathologies [40]; for example, the myocardial efflux of citrate is increased in several models of cardiomyopathy, and increasing pyruvate availability as a fuel for anaplerotic flux into the TCA cycle has shown cardioprotective effects [41].

Notwithstanding our knowledge of cardiac fuel choice in heart failure, treatment options for patients with heart failure remain poor. In addition, despite our increased knowledge of the role of different metabolic pathways in cardiac disease, the "chicken and egg" question of whether metabolism is a cause or consequence of contractile dysfunction remains largely unanswered [42]. Measuring realtime substrate metabolism in the heart in different disease-states and under different energetic demands is therefore vital to pin down the contribution of different fuels to energy generation as well as the accumulation of potentially toxic substrates, such as fatty acids and BCAAs, and to understand the causal relationship between metabolism and contractile function. It is therefore vital to understand the role of metabolism in the pathogenesis of different cardiac diseases in order to target heart disease at its core. Experimental and clinical tools to investigate metabolic fluxes and their role in pathologies are therefore crucial.

\subsection{Clinical imaging modalities to assess cardiac metabolism}

Echocardiography and cardiac magnetic resonance (CMR) are the predominant clinical imaging techniques to assess cardiac structure and function, and they are routinely employed in populationbased studies of cardiac disease [43]. Echocardiography is the cheapest and quickest way to assess cardiac function. However, it relies heavily on the skills of the sonographer and measurements can vary significantly between observers [44]; in addition, there is a systematic bias in cardiac volumes estimated via echocardiography when compared to magnetic resonance [45], [46]. CMR is a powerful 
technique that not only allows measurements of cardiac function, but also structure and tissue characterisation, such as perfusion, fibrosis and oedema [47]. Late gadolinium enhanced (LGE) magnetic resonance imaging (MRI) and $\mathrm{T}_{1}$ mapping can be used to assess tissue viability and they have prognostic value in myocardial fibrosis [48]. For these reasons, CMR represents the current gold standard of cardiac imaging [49]-[51]. Imaging techniques to measure cardiac metabolism are, however, scarce and limiting.

Positron emission tomography (PET) of radiolabelled tracers is one technique that can be used to assess cardiac metabolism in the clinic [52]. ${ }^{11} \mathrm{C}$-labelled fatty acids are employed to measure cardiac metabolism [53] and ${ }^{18} \mathrm{~F}$-fluorodeoxyglucose (FDG), which is almost the exclusively used radiolabelled probe, can be used to assess myocardial viability, inflammation and atherosclerosis [54], [55]. The main downside of PET is that it merely measures substrate uptake and retention in the tissues rather than true metabolic fluxes, as the downstream metabolic products of injected tracers are indistinguishable from their precursors. PET therefore is limited to answering questions such as whether glucose avid (FDG) or fatty-acid consuming $\left({ }^{11} \mathrm{C}\right.$-fatty acids) cells are present in the tissue of interest. Dual exploration of both fatty acid and glucose metabolism is also impossible, as the emitted photon detected from either radiolabel is essentially $511 \mathrm{keV}$, independent of the kinetic energy of the initial emitted positron, and therefore any positron-emitting tracer yields the same signal. Further PET and single photon emission computed tomography (SPECT) tracers to assess perfusion and new PET tracers to explore cardiac metabolism, such as ${ }^{11} \mathrm{C}$-methionine and fatty acid analogues, exist but are not routinely used in clinical practice [56]-[58]. Another downside of PET is that it needs an anatomic imaging technology on which to overlay the PET signal, which is routinely provided by computed tomography (CT), adding an extra radiation dose to patients. Especially for repeat measures in the paediatric patient, this poses a major long-term health concern, with a typical dose per scan of $\sim 25$ $\mathrm{mSv}$ and a $\sim 0.2-0.5 \%$ lifetime attributable increased risk of cancer [59]. In order to ameliorate this radiation hazard and simultaneously provide better soft-tissue contrast, PET scanners are now trialled in conjunction with MRI (PET/MRI), where both imaging technologies are performed either simultaneously or sequentially with the patient lying on the same table [60], [61]. This has the added advantage of being able to run MRI scans that determine anatomic structure and function with a high contrast-to-noise ratio at the same time as getting metabolic information. However, owing to the nonzero radial diameter of PET rings and the necessary room for instrumentation, PET-MR scanners often involve substantial design alterations to gradient and magnet design together with a corresponding reduction in bore size and RF body coil diameter [62].

In basic research settings, cardiac metabolic fluxes can be measured with either NMR or mass spectrometry [63] by incubating cells or injecting model organisms with ${ }^{13} \mathrm{C}$-labeled substrates and, following metabolite extraction, isotopomer analysis of the labelling pattern by either NMR or mass spectrometry [64], or via NMR performed on samples in NMR-compatible bioreactors [65]. These 
methods yield estimates of anaplerotic fluxes, for example, via the ratio of pyruvate carboxylase flux over citrate synthase flux, which is on the order of $2.5-10 \%$ in the myocardium [40]. Cardiac MR spectroscopy (MRS) can also be employed to measure metabolite levels in vivo and in patients. However, due to the poor sensitivity and low abundance of metabolites, there are great limitations in detecting metabolites with MRS and the technique is generally limited to research applications [66]. ${ }^{1} \mathrm{H}$ MRS can detect a number of metabolites, including triglycerides, lactate and total creatine (creatine plus phosphocreatine), and ${ }^{13} \mathrm{C}$ can be used to detect metabolic changes in conditions such as myocardial ischemia, heart failure and genetic cardiomyopathies [66]-[68]. Cardiac energetics can be measured in vivo with ${ }^{31} \mathrm{P}$ MRS and the ratio of phosphocreatine (PCr), the cellular ATP buffer, to ATP itself can be obtained. It has been shown that this ratio predicts mortality in patients with dilated cardiomyopathy [20], [69]. Higher magnetic field strengths and emerging technology may make it possible in the future to use MRS as a routine clinical technique to assess cardiac metabolism, and one such emerging technology, dynamic nuclear polarisation, will be discussed in detail in this review.

\subsection{Hyperpolarised ${ }^{13} \mathrm{C}$ magnetic resonance spectroscopy by dynamic nuclear polarisation}

The key limitation of MRS for the assessment of cardiac metabolism in vivo is its limited sensitivity, leading to the requirement of excessively long scan times together with the comparatively poor spatial resolution of most chemical shift imaging approaches. This inherent lack of sensitivity is created by the fact that at thermal equilibrium and physiological temperatures, the nuclear polarisation obtained at magnetic fields achievable with clinical MRI systems is only on the order of one part in a million. Hyperpolarisation methods transiently and exogenously increase the polarisation away from thermodynamic equilibrium, and hence overcome this fundamental limitation of MRS by generating an initially large magnetisation that exponentially relaxes back towards thermal equilibrium with a characteristic time, $\mathrm{T}_{1}$. As a consequence, hyperpolarised MR 'opens the door' to experimental methods that would not be feasible on signal-to-noise ratio (SNR) grounds conventionally, as by increasing the polarisation to $>10 \%$, the SNR can be increased by many orders of magnitude $(>10,000$-fold in favourable cases)[70].

Among these hyperpolarisation methods are para-hydrogen induced polarisation (PHIP) (which exploits spin isomerism in $\mathrm{H}_{2}$ often followed by polarisation transfer methods to a molecule of interest) [71]-[74], chemically induced dynamic nuclear polarisation (CIDNP) [75]-[78], spin exchange optical pumping [79]-[83], the "brute force" method (obtained by cooling a molecule to $\mathrm{mK}$ temperatures with an appropriate relaxation agent) [84]-[86], and dynamic nuclear polarisation (DNP). While DNP has been known as a method for generating spin polarised targets since 1957 [87]-[89], the more recent development of the dissolution technique enabled frozen hyperpolarised probes at $\sim 0.8-1.4 \mathrm{~K}$ to be rapidly melted and injected into living systems [90]. 
Briefly, DNP represents an ingenious method for the direct spin-pumping of nuclei of interest into a given state, under cryogenic conditions in a strong magnetic field. A source of free electrons is introduced to the sample of interest and irradiated close to its electronic Larmor frequency, which typically is at microwave frequencies. Under the conditions typically chosen $\left(B_{0} \gtrsim 3.35 T ; T<\right.$ $1.4 \mathrm{~K}$, i.e. the lowest temperature that can easily be obtained by pumped liquid helium) the electronic polarisation is nearly unity owing to the high gyromagnetic ratio of the electron and the comparatively short electronic $\mathrm{T}_{1}$ under cryogenic conditions. A (typically ${ }^{13} \mathrm{C}$ labelled) metabolite of interest under these conditions has a nuclear polarisation that is initially close to zero. By microwave irradiation near the electronic Larmor frequency, it is possible to drive simultaneous electronic and nuclear spin transitions, resulting in the initial thermal population difference between electronic spin states being transferred to an enhanced population difference between nuclear spin states. Continuous irradiation therefore enables the accumulation of nuclear polarisation, building up a substantial enhancement over the thermal value [91]. The exact mechanisms behind the hyperpolarisation process depend on the sample, source of free electrons, the geometry of the experiment and the polarisation conditions applied, and are reviewed in detail elsewhere [92], [93].

Once sufficient polarisation has built up at low temperature, the hyperpolarised compound is rapidly dissolved in a dissolution buffer, subjecting the system to a step change in temperature. The enhanced nuclear polarisation then starts to return to thermal equilibrium exponentially according to the sample's liquid-state spin-lattice relaxation time, $T_{1}$, which is typically on the order of 10-60 seconds for ${ }^{13} \mathrm{C}$-labelled metabolites dissolved in aqueous buffers. The longest $\mathrm{T}_{1}$ values are typically seen in carboxyl-group carbon nuclei as these are not directly coupled to protons, which are one major contributor to $T_{1}$ relaxation via dipole-dipole interactions. As a consequence the sample must be injected into a living system in a nearby imaging / spectrometer magnet, either manually or via an automated infusion pump [94]-[96], on a timescale that is fast or at least comparable to $\mathrm{T}_{1}$. This fact represents a significant and fundamental limitation of the technique, as all observable subsequent biochemistry has to occur on a timescale that is at least comparable to $T_{1}$. As $T_{1}$ is itself a function of magnetic field strength, the magnetic environment for this transfer should be carefully considered and, if necessary, controlled to avoid regions in which a short $T_{1}$ may destroy the enhanced polarisation far too rapidly. This has lead some groups to propose magnetic tunnels and magnetic carriers for hyperpolarised liquids that ensure that polarisation is not lost at low field through a variety of potential relaxation mechanisms that become prevalent when the Larmor frequency of the low-gamma nuclei typically used in dissolution DNP studies become comparable to that of each other, and of the proton. [97], [98] Additionally, care has to be taken to ensure that the fluid's path is free from abrupt changes in field that would rend $\partial \mathrm{B} / \partial \mathrm{t}$ comparable to the Larmor frequency, which may limit the maximum transportation rate of the sample. A dimensionless adiabaticity condition quantifying this has been proposed, namely that $\frac{1}{\gamma\left|\boldsymbol{B}^{3}\right|}\left|\boldsymbol{B} \times \frac{d \boldsymbol{B}}{d t}\right| \ll 1$ [98]. Nevertheless, despite this complexity, the use 
of hyperpolarised ${ }^{13} \mathrm{C}$-labelled substrates of central carbon metabolism allows the revolutionary detection of metabolic fluxes in vivo, as will be discussed in the following section.

\section{Cardiac applications of hyperpolarised magnetic resonance}

\subsection{Preclinical studies}

\subsubsection{Normal cardiac metabolism}

The first preclinical studies of the heart using hyperpolarised MR have probed normal cardiac metabolism using hyperpolarised pyruvate, labelled in either the first or second carbon positions which have $\mathrm{T}_{1}$ times of $\sim 60 \mathrm{~s}$ and $\sim 40 \mathrm{~s}$ respectively (Figure 2). Given the unique position of pyruvate, bridging glycolysis and oxidative phosphorylation, investigations of its metabolism can give key insight into metabolic function within cardiomyocytes. Labelling pyruvate in the first carbon position allows for monitoring of (i) its exchange with endogenous lactate, via lactate dehydrogenase (LDH); (ii) exchange with alanine via alanine aminotransferase (ALT); and (iii) formation of acetyl$\mathrm{CoA}$ via $\mathrm{PDH}$, through detection of $\mathrm{CO}_{2}$, which rapidly equilibrates with bicarbonate through carbonic anhydrase. This final conversion via PDH offers an insight into substrate selection within the heart, given the key role of PDH as a regulatory enzyme within the Randle cycle [99]. Labelling in the second carbon position, however, allows monitoring of TCA cycle flux as the labelled carbon is not lost as $\mathrm{CO}_{2}$ in the PDH-mediated reaction but enters the TCA cycle as acetyl-CoA leading to subsequent detection of citrate, glutamate and acetyl-carnitine. The last of these is thought to represent a transient storage pool for excess acetyl-CoA [100]. Hyperpolarised $\left[1-{ }^{13} \mathrm{C}\right]$ pyruvate competes with endogenous substrates for oxidation in the myocardium, and intracoronary hyperpolarised $\left[1-{ }^{13} \mathrm{C}\right]$ pyruvate concentrations of $3-6 \mathrm{mM}$ are thought to be the best target concentration in order to compete with existing substrates [101].

$\left[1-{ }^{13} \mathrm{C}\right]$ pyruvate studies have been conducted in both the isolated perfused rat heart [102] and in in vivo rodent models [103], with the probe being of great biomedical utility and additionally possessing a comparatively long carboxyl $\mathrm{T}_{1}$ lending itself well to the technique. In the in vivo rat heart, PDH flux was altered both physiologically, by overnight fasting (Figure 3), and pathologically, in type 1 
diabetes. A subsequent study correlated the ${ }^{13} \mathrm{C}$-bicarbonate labelling with PDH activity measured by enzymatic assay ex vivo in tissue extracts [104]. Furthermore, this pyruvate probe has been used to investigate the regulation of PDH in vivo, avoiding the limitations of traditional in vitro assays that do not consider key PDH regulatory parameters such as plasma substrate concentration [105]. Infusion of glucose, insulin and potassium (GIK) in fasted rats increased glucose oxidation via PDH, showing how GIK infusions can improve glucose oxidation in the heart [106]. In pigs, GIK infusion increased ${ }^{13} \mathrm{C}$-label incorporation into bicarbonate, lactate and alanine in the heart while increasing the variability in those measurements [107]. Figure 4 shows representative ${ }^{13} \mathrm{C}$ MR images of the pig heart after 120 min of GIK infusion and after hyperpolarised $\left[1-{ }^{13} \mathrm{C}\right]$ pyruvate injection. Hyperpolarised $\left[1-{ }^{13} \mathrm{C}\right]$ pyruvate can also enable detection of increased glycogen mobilisation and lactate production due to adrenergic stimulation, as shown in a perfused rat heart model treated acutely with isoproterenol [108]. Infusion of hyperpolarised $\left[1-{ }^{13} \mathrm{C}\right]$ pyruvate in the pig heart can furthermore reveal increased ${ }^{13} \mathrm{C}$-labelling of both lactate and bicarbonate reflecting increased metabolism due to angiotensin-II mediated elevated afterload [109]. Studies using $\left[1-{ }^{13} \mathrm{C}\right]$ pyruvate have also been conducted in a murine model [110], opening the door to transgenic mice studies which may aid our understanding of genetic disorders that affect cardiac metabolism [111].

$\left[2-{ }^{13} \mathrm{C}\right]$ pyruvate has also been utilised in an isolated perfused rat heart model, giving rise to ${ }^{13} \mathrm{C}$ resonances corresponding to citrate and glutamate. The citrate peak appeared before the glutamate peak, which may allow for flux calculations of the first part of the TCA cycle [112]. Following this proof of concept study, $\left[2-{ }^{13} \mathrm{C}\right]$ pyruvate has been employed to investigate the buffering role played by acetylcarnitine within the in vivo heart, which ensures sufficient acetyl-CoA to meet metabolic demand [100]. Transient storage of acetyl-CoA bound to carnitine was shown by magnetisation transfer experiments. Acetyl-CoA levels were pharmacologically increased using dichloroacetate (DCA), increasing flux through PDH, which yields higher levels of acetyl-CoA, and decreased using dobutamine, which increases workload, resulting in a $35 \%$ increase and $40 \%$ decrease in ${ }^{13} \mathrm{C}$-labelling of the acetylcarnitine pool, respectively. An understanding of this buffering mechanism as elucidated in this study may help to investigate the role of carnitine deficiency in the aetiology of heart failure [113]. [2- ${ }^{13} \mathrm{C}$ ]pyruvate can furthermore be used to measure anaplerotic flux as carboxylation to oxaloacetate and subsequent synthesis with acetyl-CoA leads to citrate labelled in the C3 position, whereas oxidative decarboxylation of pyruvate to acetyl-CoA yields citrate labelled in the C1 position. Using C3 citrate detection from hyperpolarised $\left[2-{ }^{13} \mathrm{C}\right]$ pyruvate, anaplerosis was shown to be increased in hyperthyroidism-induced cardiac hypertrophy [114].

Doubly-labelled $\left[1,2-{ }^{13} \mathrm{C}\right]$ pyruvate has also been used in combination with an interleaved acquisition scheme to assess both PDH and TCA cycle flux simultaneously [115]. This offers obvious advantages when clinical translation is considered; however, there are some limitations regarding overlapping resonances that must be addressed. Using $\left[1,2-{ }^{13} \mathrm{C}\right]$ pyruvate it was possible to determine that the 
malate-aspartate shuttle limits TCA cycle flux when cardiac workload is increased by dobutamine injection in pigs [116].

In addition to pyruvate, hyperpolarised lactate ( $\mathrm{T}_{1} \approx 45 \mathrm{~s}$ ) has also been investigated as a potential tracer [117] and possible alternative to pyruvate [118], [119]. This offers advantages over pyruvate as the plasma level of lactate is 10 times higher than that of pyruvate, and therefore there is less risk of supra-physiological levels of metabolites affecting metabolism. Similarly, hyperpolarised alanine ( $\mathrm{T}_{1} \approx 40 \mathrm{~s}$ ) has also been used to assess cardiac metabolism, and it has been shown that this offers determination of a more meaningful lactate/pyruvate ratio than using hyperpolarised pyruvate as most injected hyperpolarised pyruvate is extracellular and thus does not accurately depict intracellular levels, whereas pyruvate and lactate derived from alanine infusion are necessarily intracellular [120]. Hyperpolarised acetate $\left(\mathrm{T}_{1} \approx 16 \mathrm{~s}\right.$ ) has likewise been investigated as another method to detect in vivo TCA cycle flux into acetylcarnitine [121], [122]. Hyperpolarised butyrate ( $\mathrm{T}_{1} \approx 20 \mathrm{~s}$ ) has also been used to assess cardiac metabolism, probing short chain fatty acid oxidation and pseudoketogenesis [123]. Recently, it has been co-injected with hyperpolarised pyruvate in order to assess substrate selection within the heart, showing significant changes in substrate utilisation between the fed and fasted states as expected [124]. This method shows promise in investigating metabolic flexibility within the heart, which is often compromised in diseased states [20]. The actual ketone bodies, [1$\left.{ }^{13} \mathrm{C}\right]$ acetoacetate $\left(\mathrm{T}_{1} \approx 28 \mathrm{~s}\right)$ and $\left[1-{ }^{13} \mathrm{C}\right] \beta$-hydroxybutyrate $\left(\mathrm{T}_{1} \approx 20 \mathrm{~s}\right.$ ) have now been successfully used as hyperpolarised substrates for cardiac metabolism [125]-[127]. In perfused hearts the downstream metabolites acetylcarnitine, citrate and glutamate were detected and in in vivo rat hearts increased acetylcarnitine production from hyperpolarised $\left[1-{ }^{13} \mathrm{C}\right]$ acetoacetate was observed in the fed state [127].

\subsubsection{Cardiac metabolism in heart disease}

Table 1 shows a summary of the different cardiac pathologies studied to date in preclinical models, using hyperpolarised magnetic resonance spectroscopy.

\subsubsection{Ischaemic heart disease}

Ischaemia within the heart causes metabolic changes characterised by a decrease in oxidative phosphorylation and TCA cycle flux, and a compensatory increase in anaerobic glycolysis [128]. This glycolytic switch can be imaged using hyperpolarised $\left[1-{ }^{13} \mathrm{C}\right]$ pyruvate as PDH flux is dampened and lactate production is increased [129], [130]. Metabolic changes upon reperfusion have also been imaged using this technique, in a closed-chest coronary artery occlusion model in rats. O h-Ici and colleagues demonstrated that the lactate to pyruvate ratio increased within minutes following 
ischaemia-reperfusion but that metabolic changes normalised within a week following the ischaemic insult [131]. This study and subsequent investigations may be important in understanding reperfusion injury and may also offer a method to assess the effectiveness of reperfusion strategies. A decrease in oxidative pyruvate metabolism was subsequently shown in the same rat model of ischaemiareperfusion, and a decreased oxidative pyruvate metabolism post reperfusion modestly correlated with area at risk [132].

Other studies have utilised $\left[2-{ }^{13} \mathrm{C}\right]$ pyruvate to visualise reduced TCA cycle flux as a result of acute ischaemia/reperfusion [112], as well as longer term changes in TCA cycle flux post myocardial infarction (MI), representing an ischaemic model of chronic heart failure [133]. Ultimately, this ability to visualise ischaemia directly, in comparison to current methods which visualise ischaemia indirectly through kinetic changes during stress testing and/or perfusion defects, could aid revascularisation decision-making within the clinic. This is of particular importance in the wake of the COURAGE trial, which showed that unless the ischaemic burden of the revascularisation target area is significant, elective revascularisation does not necessarily improve outcomes [134].

An increase in anaerobic glycolysis also causes a decrease in intracellular $\mathrm{pH}$ that is characteristic of ischaemia. Initially this is beneficial as it conserves ATP by decreasing contractility; however in chronic ischaemia this sustained acidosis can damage the myocardium [135]. This change in $\mathrm{pH}$ can be imaged using $\left[1-{ }^{13} \mathrm{C}\right]$ pyruvate (Figure 5), as the ${ }^{13} \mathrm{C}$-labelled $\mathrm{CO}_{2}$ produced by $\mathrm{PDH}$ equilibrates with bicarbonate in a $\mathrm{pH}$-dependent manner and thus the bicarbonate/ $\mathrm{CO}_{2}$ ratio provides an accurate measure of intracellular $\mathrm{pH}\left(\mathrm{pH}_{\mathrm{i}}\right)$ [136]-[138]. If this visualisation of ischaemia from either lactate production or changes in $\mathrm{pH}_{\mathrm{i}}$ is coupled with an assessment of myocardial perfusion, as demonstrated by Lau et al. using non-metabolised hyperpolarised ${ }^{13} \mathrm{C}$-labeled urea $\left(\mathrm{T}_{1} \approx 20 \mathrm{~s}\right)$ as a perfusion agent [139], the functional significance of impaired perfusion can be assessed, potentially allowing clinicians to make decisions on revascularisation.

Finally, revascularisation will only be successful if the myocardium in question is viable. Currently the gold standard for viability assessment is CMR with LGE. This method was used as a reference against which to assess the ability of hyperpolarised $\left[1-{ }^{13} \mathrm{C}\right]$ pyruvate MR to determine viability. Pigs were subjected to either 15 or 45 minutes of left circumflex occlusion representing the stunned myocardium and the infarcted myocardium, respectively. The bicarbonate signal was completely absent in the infarcted group while in the stunned group the bicarbonate was decreased by $25-44 \%$, but was not abolished, indicating mitochondrial integrity and thus suggesting that the tissue was viable [140]. This therefore represents another potential clinical use of hyperpolarised MR in ischaemic heart disease. Furthermore, hyperpolarised pyruvate and its conversion to lactate can be a marker of inflammation and inflammatory macrophage infiltration in the heart post MI as shown in a rat model of cryo-infarction and in a pig model of ischaemia by left anterior descending (LAD) artery 
occlusion (Figure 6) [141]. Finally, hyperpolarised $\left[1,4-{ }^{13} \mathrm{C}_{2}\right]$ fumarate $\left(\mathrm{T}_{1} \approx 60 \mathrm{~s}\right)$ and its conversion to $\left[1,4-{ }^{13} \mathrm{C}_{2}\right]$ malate was shown to detect myocardial necrosis in the same rat cryo-MI model (Figure 7) [142], as the slow transport of fumarate compared with its $T_{1}$ means that fumarase activity is only prevalent following necrotic cell membrane rupture. Hyperpolarised $\left[1,4-{ }^{13} \mathrm{C}_{2}\right]$ fumarate has been validated as a marker of tissue necrosis in murine tumours previously [143].

\subsubsection{Non-ischaemic heart disease}

Hyperpolarised MR has also been used to examine the metabolic changes that occur in non-ischaemic heart disease. There is a growing consensus that metabolic derangement, often characterised by a change in substrate preference, may actually be a cause rather than a consequence of functional abnormalities [20]. Studies thus far have used hyperpolarised $\left[1-{ }^{13} \mathrm{C}\right]$ pyruvate to interrogate this substrate utilisation, with a favouring of fatty acid metabolism shown in a hyperthyroid model of hypertrophy [114], while a favouring of carbohydrate metabolism was observed in a spontaneously hypertensive rat (SHR) model [144]. These conflicting results may go some way to demonstrate the complex and heterogeneous phenotypes of hypertrophic heart failure [145]. It may also offer potential therapeutic opportunities, as pharmacologically enhancing PDH flux using DCA reduced levels of hypertrophy in the rodent model of hyperthyroidism-induced cardiac hypertrophy [114].

Other models of heart failure, including an abdominal aortic banding model (AAB) [146] and a pacing-induced dilated cardiomyopathy model (DCM) [147], have been investigated in order to serially depict the metabolic changes that occur in heart failure progression. In the pacing induced DCM model, hyperpolarised $\left[2{ }^{13} \mathrm{C}\right]$ pyruvate studies showed an early decrease in glutamate production, and while early on PDH flux was unaltered, decreased flux later on corresponded with the appearance of clinical features of DCM (Figure 8). It should be noted, however, that this decrease in the glutamate pool size may simply be an artefact of an elevated workload and thus decreased time available for the ${ }^{13} \mathrm{C}$ label to be transferred into the glutamate pool. Further work will be necessary to investigate whether changes in TCA cycle flux can account for the decreased PCr/ATP ratio observed in the early stages of heart failure [147]. In the AAB model, in contrast to the SHR model, no change in PDH flux was observed at any time point; however there was an increase in glycolysis, suggesting that the switch away from fatty acid oxidation may favour glycolytic metabolism rather than oxidative phosphorylation [146]. Furthermore, $\left[2-{ }^{13} C\right]$ pyruvate studies showed a decrease in the acetylcarnitine/pyruvate ratio in the group exposed to a Western diet post AAB, suggesting a reduction in the buffering capacity of the cardiomyocytes [100]. No changes in contractile function were observed, and this may again suggest that functional changes occur only once this compensated metabolism is no longer sufficient to match the energy demand. 
Diabetic cardiomyopathy has also been investigated using hyperpolarised MR. While the precise mechanism of diabetic cardiomyopathy is not fully understood, the metabolic switch to fatty acid oxidation and the subsequently decreased efficiency of ATP production is widely believed to play a major role [148]. This hypothesis has been supported in studies using $\left[1-{ }^{13} \mathrm{C}\right]$ pyruvate which have shown decreased PDH flux in both type 1 [103] and type 2 [149] diabetes. This knowledge has been used therapeutically, and DCA treatment not only restored PDH flux, but also reversed diastolic dysfunction in a rodent model of diabetes [149]. Furthermore, hyperpolarised MR has also been utilised to investigate the mechanism by which existing drugs, such as metformin, target diabetic cardiomyopathy. Lewis et al showed a direct effect on cardiac cytosolic redox state as indicated by an increased lactate/pyruvate ratio from hyperpolarised $\left[1-{ }^{13} \mathrm{C}\right]$ pyruvate [150]. In this study no functional changes were observed, and therefore future studies should investigate the use of metformin in functional disease models. This may ultimately aid the decision on whether metformin should be used to treat heart failure both in the presence and absence of diabetes [151]. L-carnitine has been thought for a long time to have many beneficial effects on cardiac metabolism in diabetes and other cardiac pathologies [14] and daily carnitine injections into type 1 diabetic rats showed increased PDH flux using hyperpolarised $\left[1-{ }^{13} \mathrm{C}\right]$ pyruvate MRS [152]. New preclinical models of heart disease are also evolving to probe different aspects of metabolism in the diseased heart. For example, hyperpolarised ${ }^{13} \mathrm{C}$ MRS has recently been employed to assess the toxic effects of chemotherapy on the heart [153], [154].

\subsection{Clinical studies}

The true goal of developing a non-invasive medical imaging technique, such as hyperpolarised MRI, lies in its translation to the clinic. The ability to provide real-time metabolic information in human subjects without delivering any ionising radiation and using naturally occurring metabolites offers obvious potential for repeat monitoring in the same subject for evaluation of disease progression or response to therapy. It also increases the potential for better understanding of normal human physiology through the study of healthy volunteers. The cardiac applications are numerous and have been reviewed elsewhere [155], [156] but include alterations in myocardial substrate selection in the failing heart, direct assessment of ischaemia through the detection of the metabolic signature of anaerobic metabolism and metabolic alterations that contribute to the pathology of a range of cardiomyopathies. Potential clinical applications also include those pathologies of infectious or other inflammatory origin. Myocarditis of viral and bacterial (rheumatic fever) origin for example can lead to sudden death or DCM [157] as well as valvular lesions [158] if not diagnosed and treated quickly. As hyperpolarised lactate imaging can detect inflammation in the heart [159] it could potentially also be used to detect myocarditis. The septic myocardium can also develop cardiomyopathy and this is 
thought to be due to mitochondrial dysfunction [160]. Understanding fuel choice and metabolic fluxes in the septic myocardium may help find a treatment to support cardiac function during sepsis, but it may also point toward treatment strategies that could benefit patients with heart disease of other aetiology.

An initial "first-in-man” study using hyperpolarised MRI was undertaken by Nelson and colleagues at the University of California, San Francisco in 2013 [161]. This study, which explored the safety and feasibility of hyperpolarised $\left[1-{ }^{13} \mathrm{C}\right]$ pyruvate as an imaging agent in patients with prostate cancer, involved the construction of a prototype hyperpolariser system in a clean room environment adjacent to the MRI scanner suite. Each dose of hyperpolarised pyruvate was produced, quality controlled and released by a pharmacist before injection into the subjects. The study demonstrated no dose-limiting toxicities up to the maximum injected dose $\left(0.43 \mathrm{ml} / \mathrm{kg}\right.$ of $230 \mathrm{mM}$ hyperpolarised $\left[1-{ }^{13} \mathrm{C}\right]$ pyruvate $)$ and showed the feasibility of imaging the conversion of $\left[1-{ }^{13} \mathrm{C}\right]$ pyruvate into $\left[1-{ }^{13} \mathrm{C}\right]$ lactate in biopsy proven prostate cancer. A subsequent study from the same group has also shown that the level of hyperpolarised $\left[1-{ }^{13} \mathrm{C}\right]$ lactate produced from $\left[1-{ }^{13} \mathrm{C}\right]$ pyruvate in the setting of prostate cancer is sensitive to the response to androgen therapy [162].

Subsequent to this work, two groups have demonstrated the feasibility of using hyperpolarised magnetic resonance imaging for cardiovascular applications. Our group in Oxford has shown that the conversion of hyperpolarised $\left[1-{ }^{13} \mathrm{C}\right]$ pyruvate into ${ }^{13} \mathrm{C}$ bicarbonate and ${ }^{13} \mathrm{CO}_{2}$, as a marker of flux through pyruvate dehydrogenase, is sensitive to the physiological modulation of cardiac metabolism that occurs in the translation from the fasted to the fed state [163]. The group of Cunningham and colleagues in Toronto has demonstrated the ability to spatially localise metabolism over the human heart [164], producing exquisite images of the metabolic conversion of $\left[1-{ }^{13} \mathrm{C}\right]$ pyruvate into [1${ }^{13} \mathrm{C}$ ]lactate and ${ }^{13} \mathrm{C}$ bicarbonate at spatial resolutions approaching those achieved by PET (Figure 9).

Whilst the initial "first-in-man" study was achieved with the use of a prototype polariser installed in a custom designed clean room adjacent to the MRI scanner, subsequent trials have been undertaken using a commercially available polariser system designed by GE Healthcare for sterile use [165]. This "SPINlab" system provides closed-cycle cryogenics that regenerate the required liquid helium overnight, a touch screen interface that simplifies operation and the ability to polarise four samples at one time. The SPINlab operates at $5 \mathrm{~T}$ and $0.8 \mathrm{~K}$, generates liquid state polarisations of up to $50 \%$ and provides $\sim 45 \mathrm{ml}$ of hyperpolarised $\left[1-{ }^{13} \mathrm{C}\right]$ pyruvate for use in approved human studies. Appropriateness for human studies is assured through the use of disposable "sterile fluid pathways" that contain the $\left[1-{ }^{13} \mathrm{C}\right]$ pyruvate, radical and buffer solutions throughout the polarisation and dissolution processes. These sterile fluid pathways can be filled within pharmacy facilities remote from the polariser and MRI system and then transported to the MRI facility for use. Following polarisation, the dissolved hyperpolarised solution undergoes a multi-parameter quality control 
process to check that the pyruvate concentration, electron radical concentration, temperature, volume, $\mathrm{pH}$ and polarisation level are all within agreed ranges before release for human injection. As of October 2017, 23 SPINlab systems have been installed in research facilities all over the world with 7 sites currently undertaking human studies and a further 6 in the process of establishing human research programs. Obviously, such studies need to be subjected to appropriate regulatory processes. Current studies in North America have been classified as clinical trials and undertaken under the regulatory scrutiny of the Food \& Drug Administration (FDA, USA) / Health Canada (Canada) and studies in the UK have been classified as physiological studies and run under the approval of the Health Research Authority.

\subsection{Acquisition techniques - challenges and strategies}

\subsubsection{Challenges}

Hyperpolarisation techniques represent a new paradigm for CMR and have the promise to enable vast new areas of study. However, in contrast to "traditional" magnetic resonance experiments performed at thermal equilibrium, hyperpolarised experiments are not typically straightforward, and, by the outof-thermodynamic equilibrium nature of the technique, need to be performed rapidly on a scale compared to the $\mathrm{T}_{1}$ of the probe in question.

Undoubtedly the greatest difference from the perspective of the experimenter is that, unlike conventional magnetic resonance, the total "amount" of signal available is non-renewable and determined by the hyperpolariser. As $T_{2} \ll T_{1}$, it is necessarily the case that every radiofrequency pulse played by the experimenter effectively destroys part of $\mathrm{M}_{0}$ in order to sample it, transferring a portion of the longitudinal magnetisation into the $x y$ plane that will decay more rapidly than if left along $z$. As a consequence, any “errant” $90^{\circ}$ pulse would effectively end a hyperpolarised experiment prematurely, sampling the entirety of $\mathrm{M}_{0}$ at one point in time. Should that pulse occur before label exchange between metabolites of interest, no useful metabolic information would be obtained from the experiment. The flip angle delivered to a particular metabolite therefore represents one of the 
paramount concerns of all hyperpolarised experiments, and consequently significant effort has been expended on optimising the "magnetisation efficiency” of hyperpolarised MR acquisitions.

To this end, it is highly desirable to have an accurate knowledge of transmit $\mathrm{B}_{1}$ (known as $\mathrm{B}_{1}{ }^{+}$) in all hyperpolarised experiments, and as ever, high $\mathrm{B}_{1}{ }^{+}$homogeneity is highly desirable. However, as all RF coils have a finite spatial extent and the $\mathrm{B}_{1}$ field near the edge of the coil is not well characterised, it is challenging to perform spin echo experiments with hyperpolarised MR unless the experiment is started after the injection of the contrast agent is complete (due to the potential effect of the ill-defined RF field on the hyperpolarised agent in the injection line). As a result, schemes involving spin echo experiments that would aim to exploit the fact that $T_{2} \gg T_{2}^{*}$ for most typical hyperpolarised compounds have to take into account the possibility that any deviation in delivered flip angle in any region containing the hyperpolarised probe, i.e. not necessarily in the region of the coil used for reception, could have significantly deleterious effects on the experiment. Consequently, the majority of pulse sequences proposed for hyperpolarised experiments are of the gradient echo, rather than spin echo, variety. For cardiac perfusion imaging, this fact represents a significant limitation, and may preclude exploiting the benefits that a very long $\mathrm{T}_{2}$ could provide for single-shot imaging in compounds such as $\left[{ }^{15} \mathrm{~N}_{2},{ }^{13} \mathrm{C}\right]$ urea [96]. Owing to the depletion of the longitudinal magnetisation by each individual excitation, experiments are usually performed with low flip angles per TR, typically $3-15^{\circ}$, with repeated acquisitions covering the temporal window that is of metabolic interest. As derived rate constants fundamentally depend on $\sin \theta$, accurate knowledge of the delivered flip angle would be required for quantitative imaging. Unfortunately, this is not straightforward owing to varying coil geometry, dielectric coupling and the inability to perform reference scans on carbon to directly measure transmit $\mathrm{B}_{1}$. This has led to a variety of different approaches for correcting for flip angle inhomogeneity, such as integrated Bloch-Siegert based reference maps acquired after a conventional imaging readout [166] to image-domain intensity normalisation techniques [167] that ameliorate some of the deleterious effects of inhomogeneity. Likewise, the desire for optimum $\mathrm{B}_{1}{ }^{+}$ and yet high sensitivity has resulted in a drive towards further optimisation of coil technology, with volume transmit/surface receive setups becoming more common and preferable to transmit/receive surface coils [168], [169], with cryo-cooling proposed for preclinical studies where the experiment is coil noise, and not sample noise, dominated [170].

Additionally, as the $\mathrm{T}_{1}$ of most agents is shortened in blood compared to water, saline or other dissolution buffers, the optimum profile for the injection of the agent into the blood stream is not known. Under most circumstances, there is a maximum practical limit on the rate of probe injection, and moreover it is desirable to retain some sort of "reservoir" of magnetisation outside of the blood pool in the initial stage of the scan. It has been shown analytically that this optimum profile is naturally itself a function of the unknown metabolic rate constants of interest, but that the commonly- 
used "boxcar" (square pulse) injection profile achieves at least $98.7 \%$ of the global optimum that a nonlinear injection scheme could achieve [171], [172].

These challenges to sequence design are further magnified by the usual problems of cardiac imaging: the heart beats continuously, and it pumps paramagnetic blood at a significant velocity. Moreover, it is anatomically located near a large number of air-tissue interfaces that cause significant susceptibility artefacts, and it contains both tissue and significant quantities of oxygenated and deoxygenated blood. In the context of working with hyperpolarisation, these susceptibility differences manifest as static $\mathrm{B}_{0}$ inhomogeneity which limits the achievable $\mathrm{T}_{2}{ }^{*}$ and, because of the preference to perform gradient echo-based acquisitions, as outlined above, reduces the time available for the imaging readout. Good shimming and excellent cardiac gating are therefore essential, and both are typically difficult to achieve.

The ideal hyperpolarised experiment therefore has to start with a clear, biologically framed question that defines the SNR envelope in which the pulse sequence has to work: for example, the desire to image the production of bicarbonate and lactate in the human or animal myocardium following injection of a given quantity of $\left[1-{ }^{13} \mathrm{C}\right]$ pyruvate possessing an initial spin polarisation of $\sim 40 \%$ on a particular imaging system. A decision about the necessary compromise between spectral, temporal and spatial resolution next has to be reached: one of the key advantages of hyperpolarised MR is that the expected behaviour of the label can be reasonably known a priori, and therefore it is possible to dramatically undersample the spectral domain based on the known chemical shift of all possible downstream metabolites for a given injected probe. Then, under these constraints, it is sensible to define a particular sequence; for example, simple slice-localised spectroscopy with a constant or variable flip angle scheme if the spectral behaviour of a novel probe is not known or spatial information is not required. An appropriate sequence can therefore be determined, and an experimental protocol planned. In other words, hyperpolarisation produces a certain amount of "cake" and it is up to the experimenter to decide how to "eat it".

\subsubsection{Strategies}

A direct consequence of these diverse and varied requirements is that a large number of pulse sequences have been proposed to interrogate the behaviour of hyperpolarised probes in vivo. It should be stressed that the majority of sequences are highly application specific and are typically optimised for the hardware that they were developed on, often in the context of relatively exotic devices such as computer-controllable infusion pumps and prototype RF coils.

\subsubsection{Spectroscopic acquisitions}


The most straightforward approach to any MR experiment is simply to obtain a pulse/acquire FID. "All" that is required for a successful acquisition is the preparation of the probe of interest, its subsequent injection into a living system, and a low flip angle (c. $10^{\circ}$ ) hard pulse followed by a, long readout acquisition with a reasonable bandwidth. Spectra are acquired with a typical TR of $\sim 1 \mathrm{~s}$, for at least $5 \times T_{1}$. Whilst pulse/acquire spectroscopy may seem a simplistic technique, it remains a robust one, requiring only approximate knowledge of the central transmitter frequency a priori, and its use lies behind a large number of important discoveries (e.g. [112], [173], [174]), with spatial localisation in these cases provided entirely through the RF coil profile.

A slight refinement is the use of variable flip angle (VFA) schemes, which progressively increase the flip angle delivered to all metabolites as time goes on, with the idea of progressively utilising remaining longitudinal magnetisation to obtain metabolite information at approximately constant SNR throughout the experiment, when otherwise their SNR would peak early and subsequently approach the noise floor. Typically, the flip angle increases monotonically from $\sim 5^{\circ}$ to $90^{\circ}$ on the final pulse, although the design of such schemes requires the solution of coupled differential equations assuming some prior information about metabolic rate constants, and is therefore not straightforward [175], [176]. Whilst such approaches can indeed bring an SNR benefit, they are highly susceptible to $B_{1}$ inhomogeneity and additionally to errors in timing between the initiation of the VFA scheme and the arrival of the hyperpolarised agent. As myocardial perfusion is itself frequently one quantity that hyperpolarised studies aim to infer, the use of a VFA scheme should be considered carefully for any planned study, and the VFA delivered must be considered in any subsequent kinetic analysis.

Moving up in complexity, the use of slice selection to localise spectroscopic acquisitions represents a straightforward extension to the technique. Again, pulse lengths can be kept short, and acquisition of 1D-localised spectra (e.g. to the heart) is relatively routine. Such approaches are simple and rapid, likely to be present as "stock" sequences on given hardware, and permit the acquisition of spectra from discontinuous locations (such as heart and liver) easily, although 1D-localisation may be insufficient to avoid multi-organ spectral contamination [177], [178]. If 3D localisation is required, single volume spectroscopic methods such as PRESS or STEAM are more challenging to apply in a hyperpolarised context due to the risk of errant high-power pulses that are nominally $180^{\circ}$ "accidentally" causing a significant reduction of signal in regions where the actual pulse length differs substantially. A modified form of LASER, localisation via adiabatic selective refocussing, has been successfully utilised in the brain, where flow is minimal and the spatial region of the coil used is well characterised [179]. Similarly, Cunningham et al. demonstrate clearly that the use of adiabatic refocussing permits 3D acquisition, but imperfectly so in regions of high flow [180], [181].

\subsubsection{Imaging acquisitions}


Traditional chemical shift imaging (CSI), in which frequency encoding is replaced by phase encoding, represents the "gold standard" tool for obtaining both spatial and spectral information about compounds present in MR experiments. However, conventional Cartesian phase encoding schemes are very slow, requiring a phase encoding step per voxel of the imaging matrix, and this effectively precludes the acquisition of high spatio-temporal resolution images. Echo planar spectroscopic imaging (EPSI), an accelerated form, is able to acquire a metabolite map following slab excitation in a period of seconds at the cost of a slightly worse point spread function, and is widely available. Both techniques are again relatively robust to centre frequency errors, and it is frequently the case that the only modification required to stock protocols is a reduction in flip angle. Consequently, owing to their wide availability and relative simplicity, CSI and EPSI sequences have been the workhorses of the early period of hyperpolarised metabolic imaging using a variety of hyperpolarised compounds, typically with a resolution on the order of $1 \mathrm{~cm}^{3}$ in small animals [182]-[185], and in prostate cancer in man [161].

While straightforward, the main disadvantage of such CSI sequences, however, is that their long acquisition time effectively limits the observation of temporal processes. Additionally, should variation exist in the injection profile of the hyperpolarised agent, the image acquired may be substantially altered. For example, if a centric-ordered CSI acquisition was planned to acquire the origin of $k$-space in the first shot, an unexpected delay in injection timing would lead to this important part of $k$-space not being acquired. As a consequence, several novel schemes have been proposed to maintain the ability to spectrally resolve different hyperpolarised metabolites, but without the long period of time that CSI techniques traditionally require. Whilst novel reconstruction methods may offer the ability to directly truncate the acquisition of spectral data from the EPSI sequence [186], [187], more conventional ways to accelerate CSI include using rapid imaging readouts, such as spiral, together with undersampling techniques such as compressed sensing [188]-[190], which effectively exploit the sparsity of MR datasets in the wavelet domain to infer data not acquired. Note that accelerating the trajectory of CSI acquisitions can come at a cost in either SNR or the ultimate point spread function of the acquired data [191].

An alternative approach exploits the $a$ priori knowledge the experimenter has about which metabolic pathways a given injected hyperpolarised probe is likely to traverse. The location of resonances of interest is therefore easily determined ahead of the experiment, and the spectral domain can be dramatically under sampled. A popular technique for exploiting this sparsity is IDEAL CSI, in which a conventional spatially selective excitation with a spectral bandwidth sufficient to excite each of the $n$ expected metabolites is applied, and an 'ordinary' rapid imaging readout sequence (EPI, spiral) applied with $n$ different echo times. A Fourier operator is then constructed based on the known chemical shift of each metabolite, and the data deconvolved to produce a set of $n$ images each of an individual metabolite [192]. As the excitation is rapid, this technique can achieve high spatial 
resolution in-plane, and has enjoyed success in the anaesthetised rodent heart with numerous probes, such as $\left[1-{ }^{13} \mathrm{C}\right]$ pyruvate, $\left[1-{ }^{13} \mathrm{C}\right]$ acetate and ${ }^{13} \mathrm{C}$-acetylcarnitine [193]-[195].

Another profitable area for metabolic imaging is the use of spectrally and spatially selective excitation, followed by a conventional rapid imaging readout. Spectral-Spatial RF pulses were originally developed in the context of fat/water imaging, and have found considerable utility in hyperpolarised studies [196]-[202]. Such pulses consist of a rapidly oscillating slice-select gradient train with a "comb" of radiofrequency pulses played out under a shaped envelope. An exact excitation $\mathrm{k}$-space formalism exists for the development of such pulses, and they are typically designed to excite a single resonance lying within a particular frequency band within a given slice thickness [203]. The finite length of the excitation causes periodicity in the resulting excitation profile, leading to the concept of a "stopband" and "passband" between each excited "island" of magnetisation. By judiciously lining up the spectral location of these "islands" with those of the injected compound, it is possible to independently deliver different flip angles to different metabolites. This can be highly magnetisation efficient: the magnetisation of the injected probe can be left barely perturbed, and unidirectionally produced downstream metabolites can be imaged with a high flip angle, and thus high SNR, assuming that they are replenished within the timescale of the experiment. In practice, spectralspatial excitation enables high resolution bicarbonate imaging following the injection of hyperpolarised pyruvate in the human myocardium [204]. It should be noted that spiral-out and centric-ordered trajectories are well suited to spectral-spatial excitation, as one reads "out" into the available $k$-space, obtaining resolution for as long as the achievable $\mathrm{T}_{2}{ }^{*}$ permits.

Spectral-spatial approaches are, however, highly reliant upon accurate knowledge a priori of the centre frequency of the metabolite of interest. Therefore, independent of the imaging readout used, they require high $\mathrm{B}_{0}$ homogeneity; a mis-registration between the transmitter frequency and the actual frequency of the labelled hyperpolarised probe of interest would be sufficient to preclude its excitation, or that of its downstream metabolic products. Moreover, the small imaging bandwidth and demanding acquisition environment of the heart often makes single-shot imaging challenging and, unlike other spectroscopic means of imaging, these problems often appear as a discrepancy between the $k$-space trajectory intended and that actually taken. Numerous algorithms have been proposed to ameliorate these errors, which necessarily depend on the underlying trajectory - e.g. susceptibility effects cause translations and shearing artefacts in EPI which can be ameliorated by alternating the direction in which k-space is traversed [205]-[207]; but they cause blurring and nonlinear distortions with spiral trajectories [208], [209]. Likewise, spectral-spatial pulses are typically designed for highresolution imaging of a particular metabolic probe on a particular individual MR system. As the achievable slice thickness is limited by gradient duty cycle, maximum strength, and fidelity, nonlinear distortions readily become apparent in the excitation profile of the spectral-spatial pulse. As first pointed out by Sigfridsson, such limitations effectively place an upper limit on using the 
technique in a multiple-slice approach [210]. As a consequence, 3D selective excitation has been proposed with phase encoding through the z-direction to trade temporal for spatial resolution, optionally with the use of compressed sensing to reduce the effective number of (time-consuming) phase encodes required, with both EPI and spiral trajectories [211]-[213]. Moreover, the 3D approach can be shown analytically to be SNR optimal compared to multi-slice approaches under certain conditions, although the non-renewable magnetisation causes a trade-off between through-slice blurring and SNR [214], [215].

One further, novel approach taken is the use of multiband excitation schemes, whereby multiple resonances are excited simultaneously, but with differing flip angles [142], [210], [216], [217]. Standard CSI techniques can then subsequently be used as imaging readouts, and images obtained with approximately equal SNR between the observed metabolites. Likewise, owing to the Fourier relationships inherent in MR, such schemes typically require a short RF excitation to hit different metabolites with appropriate flip angles. Such short RF pulses (and associated gradient trains) place substantially lower demands on gradient hardware than the majority of spectral-spatial pulses. A multi-echo readout can therefore reconstruct an image of each excited metabolite, and is particularly well suited for moieties with a short $T_{2}$ where minimising the echo time is important [142]. This approach, however, is susceptible to frequency errors, which again would correspond to errors in the delivered flip angle, potentially utilising the entirety of the magnetisation in the injected probe prematurely by errantly playing a high-flip angle pulse. Another 'unconventional' approach to RF pulse design for hyperpolarised imaging is the use of simultaneous multi-slice techniques to excite up to three slices simultaneously, reading out aliased images that can be reconstructed through the use of differing coil sensitivities, provided that the hardware used permits such approaches [218].

An alternative approach, designed to be more tolerant of field inhomogeneities that might cause variation in flip angle, is the use of spatiotemporal encoding (SPEN) techniques that rely upon the selective excitation of particular hyperpolarised moieties via a technically distinct class of multidimensional excitation. SPEN excitation requires a frequency-swept pulse applied in the presence of an encoding gradient, followed by its later removal using an acquisition gradient; this technique is therefore not necessarily subject to the same ultimate hardware limitations in slice thickness and spectral separation as spectral-spatial excitation [219]-[221]. One can likewise relegate the requirement for spatial localisation to the RF coil, and design a short, spectrally selective RF pulse with the aim of then performing a 3D readout across different metabolites sampled individually. This approach has been used to reconstruct maps of myocardial $\mathrm{pH}$ in the rodent heart, by measuring the production of hyperpolarised carbon dioxide and bicarbonate following the injection of [1${ }^{13} \mathrm{C}$ ]pyruvate [222]. 
Finally, it is worth noting that hyperpolarised perfusion agents do not require the separation of both chemical and spatial information to be of utility [223], [224]. Consequently, traditional gradient echo single-shot imaging techniques, such as spiral or echo-planar acquisitions may be applied, with a low flip angle. In addition, approaches have been reported using balanced "steady-state" free procession type approaches, whereby successive flip angles are chosen to routinely keep the magnetisation vector close to $M_{z}$, and a variable flip angle scheme utilised to maximise the effective SNR whilst simultaneously obtaining endogenous $\mathrm{T}_{2}$ contrast with the hyperpolarised probe [225]-[228].

It is worth stressing the value of simplicity in experiments involving hyperpolarisation: more sophisticated experiments can offer the promise of more information, but often the $\mathrm{B}_{0}, \mathrm{~B}_{1}$, timing and frequency requirements that they necessarily set are challenging to routinely realise in practice and are highly optimised for the specific purposes for which they were developed. As a consequence, these diverse sequences often require a significant amount of preparation to enable their correct function, for example per-subject ${ }^{13} \mathrm{C}$ transmit power calibrations, and the use of reference phantoms together with cardiac-gated $\mathrm{B}_{0}$ maps to enable accurate calculation of the central transmitter frequency required [213].

However, even given these caveats, hyperpolarised pulse sequences have the promise to image and potentially quantify a vast number of parameters of interest: including myocardial perfusion, $\mathrm{pH}$ and metabolic rate constants following the injection of a given probe, as summarised in Table 2. As ever, the pulse sequence chosen should be constructed solely in the context of a well-posed and relevant biological question.

\section{Future applications of cardiac hyperpolarised magnetic resonance spectroscopy}

\subsection{Hyperpolarised substrates for potential use in cardiac applications}

Many more substrates have been used for hyperpolarised MRS in preclinical models and in proof-ofconcept studies than those employed in applications for cardiology [229]. Hyperpolarised [U-2 $\mathrm{H}$, U${ }^{13} \mathrm{C}$ ]glucose for example is a marker of glycolysis [230] and the pentose phosphate pathway (PPP) [231] in tumours. Hyperpolarised glucose is a more direct marker of glycolytic flux than pyruvate; however, its short $\mathrm{T} 1$ relaxation time $\left(\mathrm{T}_{1} \approx 9 \mathrm{~s}\right)$ and low downstream metabolite signals due to peaksplitting by ${ }^{13} \mathrm{C}-{ }^{13} \mathrm{C}$ J-coupling currently prohibit its use in the heart as well as its translation into the clinic. However, recent synthesis of deuterated glucose selectively carbon-13 labelled in the C3 and C4 positions now allows detection as singlets of downstream metabolites (in which the C3-C4 bond 
has been cleaved), which improves signal-to-noise ratio in ${ }^{13} \mathrm{C}$ spectra and may make hyperpolarised glucose measurements in the heart possible [232]. Similarly, hyperpolarised $\left[1-{ }^{13} \mathrm{C}\right]$ glycerate $\left(\mathrm{T}_{1} \approx 30\right.$ s) has been proposed as a new marker of glycolysis [233], $\delta$-[1- $\left.{ }^{13} \mathrm{C}\right]$ gluconolactone $\left(\mathrm{T}_{1} \approx 20 \mathrm{~s}\right)$ as a substrate for the PPP [234] and hyperpolarised $\left[2-{ }^{13} \mathrm{C}\right]$ dihydroxyacetone $\left(\mathrm{T}_{1} \approx 30 \mathrm{~s}\right)$ as a marker of both glycolysis and gluconeogenesis in the liver and kidney [235], [236]. PPP flux is increased in oxidative stress as it produces NADPH, which keeps the major cytosolic antioxidant, glutathione, in the reduced state [237]. Measuring PPP flux in the heart could therefore be of utility in cardiac pathologies associated with oxidative stress, such as chemotherapy-induced cardiotoxicity [238], ischemia and heart failure [239]. Hyperpolarised $\left[1-{ }^{13} \mathrm{C}\right]$ dehydroascorbic acid $\left(\mathrm{T}_{1} \approx 20 \mathrm{~s}\right)$ is a marker of redox state and could be an interesting substrate with which to assess myocardial redox capacity in oxidative stress [240]-[242]. Hyperpolarised $\left[1-{ }^{13} \mathrm{C}\right]$ glutamate $\left(\mathrm{T}_{1} \approx 34 \mathrm{~s}\right)$ has been used to detect anaplerotic flux into the TCA cycle by labelling $\alpha$-ketoglutarate [243]. BCAA metabolism can be imaged in tumours with hyperpolarised ${ }^{13} \mathrm{C}$ ketoisocaproate [244] ( $\mathrm{T}_{1} \approx 55 \mathrm{~s}$ ). The medium chain fatty acid octanoate was employed in one study to assess myocardial $\beta$-oxidation and the injection into a rat led to myocardial production of acetate and acetyl-carnitine [245]. However, to this day no long-chain fatty acid probe has been used for hyperpolarisation. Long chain-fatty acids as hyperpolarised substrates would be a valuable tool to truly measure fatty acid oxidation in different disease states. However, the very short $\mathrm{T}_{1}$ relaxation times ( 1-5 s) of fatty acid carbon nuclei prevent their use in hyperpolarised MRS at this stage. Extracellular pH can be measured with hyperpolarised bicarbonate [246] and recently a few new markers of intracellular pH have been developed [247], [248]. Substrates can also be co-polarised to assess different aspects of biology, such as lactate export and necrosis with co-polarised pyruvate and fumarate [249]. Lastly, silicon nanoparticles have recently been hyperpolarised to image perfusion [250]. As ${ }^{29} \mathrm{Si}$-particles are biocompatible, biodegradable, show flexible surface chemistry that allow tagging and have very long spin-lattice relaxation times that are a function of their radius, they could potentially be useful markers for hyperpolarised imaging.

\subsection{Improving and extending substrates for hyperpolarised MRI}

The major limitations of hyperpolarised MRI are the polarisation levels with a given molecule, delivery and uptake of substrates, $\mathrm{T}_{1}$, metabolite turn-over and reproducibility of the obtained results. Higher field strength hyperpolarisers could lead to higher polarisation levels [251], which may push some substrates to polarisation levels sufficient for in vivo applications. Automated injection systems can reduce the dissolution-to-injection time and standardise injection volume [94]. Direct arterial injections of hyperpolarised substrates into rat tumours have also been trialled to image metabolism after injection more rapidly [252]. Alterations in substrate chemistry can make the use of otherwise cell-impermeable substrates possible or allow the delivery of substrates to the brain that are normally retained by the blood brain barrier [253]. Derivatisation of hyperpolarised substrates to their ethyl- or 
methyl-esters, for example, can make substrates cell permeable and intracellular esterases rapidly cleave the ester group off to liberate the active metabolite. Likewise, $\mathrm{T}_{1}$ can be increased by deuteration of the metabolite, which prevents relaxation of ${ }^{13} \mathrm{C}$ nuclei by neighbouring protons [254]. Preservation of the nuclear polarisation by exploiting singlet-triplet splitting and trapping magnetisation in a singlet state can also significantly increase $T_{1}$ [255], [256]. Low levels of hyperpolarisation have now also been achieved without the addition of an organic free radical, by inducing transient radical formation from pyruvic acid itself through UV-mediated photochemistry. [257]. Sample preparation in this way circumvents subsequent filtration of free radicals for human injections, which could reduce the dissolution-to-injection time in man. Substrates hyperpolarised by UV irradiation have been successfully injected into rats and their metabolism assessed in the myocardium [258]. This technique has now been extended to thermally annihilate the radicals and store the frozen polarised pellets for hours before dissolving and injecting substrates into living systems [259]. Most hyperpolarised substrates take several hours to polarise, limiting the number of experiments that can be performed in a day. The clinical SPINlab system can hyperpolarise four samples at the same time [165] and preclinical equivalents for small animal sample polarisation have now also been built [260], [261]. A further point of consideration is reproducibility of the obtained hyperpolarised data. Maintaining the depth of anaesthesia is, for example, a vital requirement, as isoflurane concentration has been shown to affect cardiac metabolism in healthy rats [262]. Lastly, more metabolic information can be obtained by interfacing an MRI scanner capable of ${ }^{13} \mathrm{C}$ hyperpolarised MR detection with a PET scanner (hyperPET) [263]-[265].

\section{Conclusions}

Hyperpolarised ${ }^{13} \mathrm{C}$ MRS is unique in its function to measure metabolic fluxes in real-time in vivo. Numerous preclinical studies have shown its benefit in delineating fuel choices and assessing the efficacy of drugs in models of human disease. It will be exciting to see over the next few years how those findings translate into clinical trials with hyperpolarised MRI and how this fascinating technique may change and improve patient care.

\section{Acknowledgements}

KNT, JJM and DJT would like to acknowledge the financial support provided by a British Heart Foundation Immediate Postdoctoral Fellowship; Novo Nordisk Postdoctoral Fellowship; and a British Heart Foundation Senior Fellowship respectively (KNT: FS/16/7/31843, DJT: FS/14/17/30634). All 
authors would like to acknowledge the support provided by the OXFORD-BHF Centre for Research Excellence (grant RE/13/1/30181) and the National Institute for Health Research Oxford Biomedical Research Centre programme. 


\section{References}

[1] J. E. Baldwin and H. Krebs, "The evolution of metabolic cycles,” Nature, vol. 291, no. 5814, pp. 381-382, 1981.

[2] Ingwall, ATP and the heart. Boston: Kluwer Academic, 2002.

[3] D. J. Fisher, “Oxygenation and metabolism in the developing heart,” Semin Perinatol, vol. 8, no. 3, pp. 217-225, 1984.

[4] G. D. Lopaschuk, D. D. Belke, J. Gamble, T. Itoi, and B. O. Schonekess, "Regulation of fatty acid oxidation in the mammalian heart in health and disease," Biochim Biophys Acta, vol. 1213, no. 3, pp. 263-276, 1994.

[5] G. D. Lopaschuk, J. R. Ussher, C. D. L. Folmes, J. S. Jaswal, and W. C. Stanley, "Myocardial Fatty Acid Metabolism in Health and Disease," Physiol. Rev., vol. 90, no. 1, pp. 207-258, 2010.

[6] P. J. Randle, P. B. Garland, C. N. Hales, and E. A. Newsholme, "The glucose fatty-acid cycle. Its role in insulin sensitivity and the metabolic disturbances of diabetes mellitus," Lancet, vol. 1, no. 7285, pp. 785-789, 1963.

[7] L. Hue and H. Taegtmeyer, "The Randle cycle revisited: a new head for an old hat,” Am J Physiol Endocrinol Metab, vol. 297, no. 3, pp. E578-91, 2009.

[8] D. Shao and R. Tian, "Glucose transporters in cardiac metabolism and hypertrophy," Compr. Physiol., vol. 6, no. 1, pp. 331-351, 2016.

[9] R. Malhotra and F. C. Brosius, "Glucose uptake and glycolysis reduce hypoxia-induced apoptosis in cultured neonatal rat cardiac myocytes.," J. Biol. Chem., vol. 274, no. 18, pp. 12567-75, 1999.

[10] E. W. Kraegen, J. a Sowden, M. B. Halstead, P. W. Clark, K. J. Rodnick, D. J. Chisholm, and D. E. James, "Glucose transporters and in vivo glucose uptake in skeletal and cardiac muscle: fasting, insulin stimulation and immunoisolation studies of GLUT1 and GLUT4.," Biochem. J., vol. 295 ( Pt 1, no. 1993, pp. 287-293, 1993.

[11] J. E. Dimsdale and J. A. Herd, "Variability of plasma lipids in response to emotional arousal," Psychosomatic Medicine, vol. 44, no. 5. pp. 413-430, 1982.

[12] G. Boden, “Obesity, insulin resistance and free fatty acids.," Curr Opin Endocrinol Diabetes Obes, vol. 18, no. 2, pp. 139-43, 2011.

[13] P. D. Berk and D. D. Stump, "Mechanisms of cellular uptake of long chain free fatty acids.," Mol. Cell. Biochem., vol. 192, no. Box 1633, pp. 17-31, 1999.

[14] Z.-Y. Wang, Y.-Y. Liu, G.-H. Liu, H.-B. Lu, and C.-Y. Mao, "L-Carnitine and heart disease," Life Sci., 2017.

[15] World Health Organisation, World Health Statistics 2015. 2015.

[16] P. K. Mazumder, B. T. O’Neill, M. W. Roberts, J. Buchanan, U. J. Yun, R. C. Cooksey, S. Boudina, and E. D. Abel, "Impaired cardiac efficiency and increased fatty acid oxidation in insulin-resistant ob/ob mouse hearts,” Diabetes, vol. 53, no. 9, pp. 2366-2374, 2004.

[17] S. C. Kolwicz Jr. and R. Tian, "Glucose metabolism and cardiac hypertrophy,” Cardiovasc Res, vol. 90, no. 2, pp. 194-201, 2011.

[18] M. Kerr, M. S. Dodd, and L. C. Heather, "The 'Goldilocks zone' of fatty acid metabolism; to ensure that the relationship with cardiac function is just right," Clin. Sci., vol. 131, no. 16, pp. 2079-2094, 2017. 
[19] W. G. Hunter, J. P. Kelly, R. W. McGarrah, W. E. Kraus, and S. H. Shah, "Metabolic Dysfunction in Heart Failure: Diagnostic, Prognostic, and Pathophysiologic Insights From Metabolomic Profiling,” Current Heart Failure Reports, vol. 13, no. 3. pp. 119-131, 2016.

[20] S. Neubauer, “The failing heart--an engine out of fuel,” N Engl J Med, vol. 356, no. 11, pp. 1140-1151, 2007.

[21] F. Pascual and R. A. Coleman, "Fuel availability and fate in cardiac metabolism: A tale of two substrates," Biochimica et Biophysica Acta - Molecular and Cell Biology of Lipids, vol. 1861, no. 10. pp. 1425-1433, 2016.

[22] S. C. Kolwicz Jr. and R. Tian, "Metabolic therapy at the crossroad: how to optimize myocardial substrate utilization?,” Trends Cardiovasc Med, vol. 19, no. 6, pp. 201-207, 2009.

[23] H. Noordali, B. L. Loudon, M. P. Frenneaux, and M. Madhani, "Cardiac metabolism - A promising therapeutic target for heart failure,” Pharmacol. Ther., 2017.

[24] A. N. Carley, H. Taegtmeyer, and E. D. Lewandowski, "Matrix revisited: mechanisms linking energy substrate metabolism to the function of the heart," Circ Res, vol. 114, no. 4, pp. 717729, 2014.

[25] W. H. Barry, "Heart Physiology From Cell to Circulation, 4th ed.," Circulation, vol. 110, no. 12, pp. e313-e313, 2004.

[26] H. Noordali, B. L. Loudon, M. P. Frenneaux, and M. Madhani, "Cardiac metabolism - A promising therapeutic target for heart failure,” Pharmacol. Ther., vol. 182, 2018.

[27] C. R. Chong, B. Sallustio, and J. D. Horowitz, "Drugs that Affect Cardiac Metabolism: Focus on Perhexiline,” Cardiovasc. Drugs Ther., vol. 30, no. 4, pp. 399-405, 2016.

[28] H. Zou, X.-X. Zhu, Y.-H. Ding, Q.-Y. Jin, L.-Y. Qian, D.-S. Huang, and X.-J. Cen, "Trimetazidine in conditions other than coronary disease, old drug, new tricks?.," Int. J. Cardiol., vol. 234, pp. 1-6, 2017.

[29] D. A. vi. W. Crabb, E. A. Yount, and R. A. Harris, "The metabolic effects of dichloroacetate," Metabolism, vol. 30, no. 10, pp. 1024-1039, 1981.

[30] G. D’Antona, M. Ragni, A. Cardile, L. Tedesco, M. Dossena, F. Bruttini, F. Caliaro, G. Corsetti, R. Bottinelli, M. O. Carruba, A. Valerio, and E. Nisoli, "Branched-chain amino acid supplementation promotes survival and supports cardiac and skeletal muscle mitochondrial biogenesis in middle-aged mice,” Cell Metab., vol. 12, no. 4, pp. 362-372, 2010.

[31] T. Li, Z. Zhang, S. C. Kolwicz, L. Abell, N. D. Roe, M. Kim, B. Zhou, Y. Cao, J. Ritterhoff, H. Gu, D. Raftery, H. Sun, and R. Tian, "Defective Branched-Chain Amino Acid Catabolism Disrupts Glucose Metabolism and Sensitizes the Heart to Ischemia-Reperfusion Injury,” Cell Metab., vol. 25, no. 2, pp. 374-385, 2017.

[32] H. Sun, K. C. Olson, C. Gao, D. A. Prosdocimo, M. Zhou, Z. Wang, D. Jeyaraj, J. Y. Youn, S. Ren, Y. Liu, C. D. Rau, S. Shah, O. Ilkayeva, W. J. Gui, N. S. William, R. M. Wynn, C. B. Newgard, H. Cai, X. Xiao, D. T. Chuang, P. C. Schulze, C. Lynch, M. K. Jain, and Y. Wang, "Catabolic defect of branched-chain amino acids promotes heart failure," Circulation, vol. 133, no. 21, pp. 2038-2049, 2016.

[33] Y. Tanada, T. Shioi, T. Kato, A. Kawamoto, J. Okuda, and T. Kimura, "Branched-chain amino acids ameliorate heart failure with cardiac cachexia in rats,” Life Sci., vol. 137, pp. 20-27, 2015.

[34] J. A. Pineda-Juárez, N. A. Sánchez-Ortiz, L. Castillo-Martínez, A. Orea-Tejeda, R. CervantesGaytán, C. Keirns-Davis, C. Pérez-Ocampo, K. Quiroz-Bautista, M. Tenorio-Dupont, and A. Ronquillo-Martínez, "Changes in body composition in heart failure patients after a resistance 
exercise program and branched chain amino acid supplementation,” Clin. Nutr., vol. 35, no. 1, pp. 41-47, 2016.

[35] M. Takata, E. Amiya, M. Watanabe, Y. Hosoya, A. Nakayama, T. Fujiwara, M. Taya, G. Oguri, K. Hyodo, N. Takayama, N. Takano, T. Mashiko, Y. Uemura, and I. Komuro, “An exploratory study on the efficacy and safety of a BCAA preparation used in combination with cardiac rehabilitation for patients with chronic heart failure,” BMC Cardiovasc. Disord., vol. 17, no. 1, p. 205, 2017.

[36] D. G. Cotter, R. C. Schugar, and P. A. Crawford, "Ketone body metabolism and cardiovascular disease,” Am J Physiol Hear. Circ Physiol, vol. 304, no. 8, pp. H1060-76, 2013.

[37] M. Evans, K. E. Cogan, and B. Egan, "Metabolism of ketone bodies during exercise and training: physiological basis for exogenous supplementation,” Journal of Physiology, vol. 595, no. 9. pp. 2857-2871, 2017.

[38] P. J. Cox, T. Kirk, T. Ashmore, K. Willerton, R. Evans, A. Smith, A. J. Murray, B. Stubbs, J. West, S. W. McLure, M. T. King, M. S. Dodd, C. Holloway, S. Neubauer, S. Drawer, R. L. Veech, J. L. Griffin, and K. Clarke, "Nutritional Ketosis Alters Fuel Preference and Thereby Endurance Performance in Athletes,” Cell Metab., vol. 24, no. 2, pp. 256-268, 2016.

[39] A. J. Murray, N. S. Knight, M. A. Cole, L. E. Cochlin, E. Carter, K. Tchabanenko, T. Pichulik, M. K. Gulston, H. J. Atherton, M. A. Schroeder, R. M. J. Deacon, Y. Kashiwaya, M. T. King, R. Pawlosky, J. N. P. Rawlins, D. J. Tyler, J. L. Griffin, J. Robertson, R. L. Veech, and K. Clarke, "Novel ketone diet enhances physical and cognitive performance," FASEB J., vol. 30, no. 12, pp. 4021-4032, 2016.

[40] C. Des Rosiers, F. Labarthe, S. G. Lloyd, and J. C. Chatham, "Cardiac anaplerosis in health and disease: food for thought,” Cardiovasc Res, vol. 90, no. 2, pp. 210-219, 2011.

[41] M. J. Gibala, M. E. Young, and H. Taegtmeyer, "Anaplerosis of the citric acid cycle: role in energy metabolism of heart and skeletal muscle,” Acta Physiol. Scand., vol. 168, no. 4, pp. 657-665, 2000.

[42] H. Taegtmeyer and G. Lubrano, "Rethinking cardiac metabolism: metabolic cycles to refuel and rebuild the failing heart,” F1000Prime Rep, vol. 6, p. 90, 2014.

[43] T. H. Marwick, S. Neubauer, and S. E. Petersen, "Use of cardiac magnetic resonance and echocardiography in population-based studies: Why, where, and when?," Circ. Cardiovasc. Imaging, vol. 6, no. 4, pp. 590-596, 2013.

[44] O. Strohm, J. Schulz-Menger, B. Pilz, K. J. Osterziel, R. Dietz, and M. G. Friedrich, "Measurement of left ventricular dimensions and function in patients with dilated cardiomyopathy,” J Magn Reson Imaging, vol. 13, no. 3, pp. 367-371, 2001.

[45] R. M. Lang, L. P. Badano, V. Mor-Avi, J. Afilalo, A. Armstrong, L. Ernande, F. A. Flachskampf, E. Foster, S. A. Goldstein, T. Kuznetsova, P. Lancellotti, D. Muraru, M. H. Picard, E. R. Rietzschel, L. Rudski, K. T. Spencer, W. Tsang, and J.-U. Voigt, "Recommendations for Cardiac Chamber Quantification by Echocardiography in Adults: An Update from the American Society of Echocardiography and the European Association of Cardiovascular Imaging,” Eur. Hear. J. - Cardiovasc. Imaging, vol. 16, no. 3, pp. 233-271, Mar. 2015.

[46] M. Whitlock, A. Garg, J. Gelow, T. Jacobson, and C. Broberg, "Comparison of left and right atrial volume by echocardiography versus cardiac magnetic resonance imaging using the arealength method.,” Am. J. Cardiol., vol. 106, no. 9, pp. 1345-50, 2010.

[47] M. Salerno, B. Sharif, H. Arheden, A. Kumar, L. Axel, D. Li, and S. Neubauer, "Recent Advances in Cardiovascular Magnetic Resonance," Circ. Cardiovasc. Imaging, vol. 10, no. 6, 
2017.

[48] B. Ambale-Venkatesh and J. A. C. Lima, "Cardiac MRI: A central prognostic tool in myocardial fibrosis,” Nature Reviews Cardiology, vol. 12, no. 1. pp. 18-29, 2015.

[49] J. Post, A. van Rossum, J. B.- Circulation, and U. 1995, "Magnetic resonance angiography of anomalous coronary arteries,” Am Hear. Assoc.

[50] W. O. Ntim and W. G. Hundley, "Cardiac Imaging in Patients With Chronic Obstructive Pulmonary Disease and Chronic Heart Failure,” J. Am. Coll. Cardiol., vol. 49, no. 18, pp. 1900-1901, 2007.

[51] M. Whitlock, A. Garg, J. Gelow, T. Jacobson, and C. Broberg, "Comparison of left and right atrial volume by echocardiography versus cardiac magnetic resonance imaging using the arealength method.," Am. J. Cardiol., vol. 106, no. 9, pp. 1345-50, 2010.

[52] V. Dilsizian, S. L. Bacharach, R. S. Beanlands, S. R. Bergmann, D. Delbeke, S. Dorbala, R. J. Gropler, J. Knuuti, H. R. Schelbert, and M. I. Travin, "ASNC imaging guidelines/SNMMI procedure standard for positron emission tomography (PET) nuclear cardiology procedures,” J. Nucl. Cardiol., vol. 23, no. 5, pp. 1187-1226, 2016.

[53] S. R. Bergmann, C. J. Weinheimer, J. Markham, and P. Herrero, "Quantitation of myocardial fatty acid metabolism using PET.,” J. Nucl. Med., vol. 37, no. 10, pp. 1723-30, 1996.

[54] P. Joseph and A. Tawakol, "Imaging atherosclerosis with positron emission tomography,” European Heart Journal, vol. 37, no. 39. p. 2974-2980b, 2016.

[55] M. Miyagawa, R. Yokoyama, Y. Nishiyama, A. Ogimoto, J. Higaki, and T. Mochizuki, "Positron Emission Tomography-Computed Tomography for Imaging of Inflammatory Cardiovascular Diseases,” Circ. J., vol. 78, no. 6, pp. 1302-1310, 2014.

[56] O. Manabe, T. Kikuchi, A. J. H. A. Scholte, M. El Mahdiui, R. Nishii, M. R. Zhang, E. Suzuki, and K. Yoshinaga, "Radiopharmaceutical tracers for cardiac imaging,” J. Nucl. Cardiol., pp. 1-33, 2017.

[57] M. Kircher and C. Lapa, "Novel Noninvasive Nuclear Medicine Imaging Techniques for Cardiac Inflammation,” Curr. Cardiovasc. Imaging Rep., vol. 10, no. 2, 2017.

[58] M. Osterholt, S. Sen, V. Dilsizian, and H. Taegtmeyer, "Targeted metabolic imaging to improve the management of heart disease,” JACC Cardiovasc Imaging, vol. 5, no. 2, pp. 214 226, 2012.

[59] B. Huang, M. W.-M. Law, and P.-L. Khong, "Whole-body PET/CT scanning: estimation of radiation dose and cancer risk.,” Radiology, vol. 251, no. 1, pp. 166-74, Apr. 2009.

[60] P. M. Robson, D. Dey, D. E. Newby, D. Berman, D. Li, Z. A. Fayad, and M. R. Dweck, "MR/PET Imaging of the Cardiovascular System,” JACC: Cardiovascular Imaging, vol. 10, no. 10. pp. 1165-1179, 2017.

[61] C. Rischpler and S. G. Nekolla, "PET/MR Imaging in Heart Disease,” PET Clinics, vol. 11, no. 4. pp. 465-477, 2016.

[62] H. Zaidi and A. Del Guerra, “An outlook on future design of hybrid PET/MRI systems,” Med. Phys., vol. 38, no. 10, pp. 5667-5689, Sep. 2011.

[63] J. L. Griffin, H. Atherton, J. Shockcor, and L. Atzori, "Metabolomics as a tool for cardiac research,” Nat Rev Cardiol, vol. 8, no. 11, pp. 630-643, 2011.

[64] C. Des Rosiers and J. C. Chatham, "Myocardial phenotyping using isotopomer analysis of metabolic fluxes,” Biochem. Soc. Trans., vol. 33, no. Pt 6, pp. 1413-1417, 2005. 
[65] R. J. Gillies, J. -P Galons, K. A. McGovern, P. G. Scherer, Y. -H Lien, C. Job, R. Ratcliff, F. Chapa, S. Cerdan, and B. E. Dale, "Design and application of NMR-compatible bioreactor circuits for extended perfusion of high-density mammalian cell cultures," NMR Biomed., vol. 6, no. 1, pp. 95-104, Jan. 1993.

[66] C. J. Holloway, J. Suttie, S. Dass, and S. Neubauer, "Clinical Cardiac Magnetic Resonance Spectroscopy,” Prog. Cardiovasc. Dis., vol. 54, no. 3, pp. 320-327, 2011.

[67] W. T. Qureshi and U. bin Nasir, "Principals and clinical applications of magnetic resonance cardiac spectroscopy in heart failure," Heart Failure Reviews, vol. 22, no. 4. pp. 491-499, 2017.

[68] P. A. van Ewijk, V. B. Schrauwen-Hinderling, S. C. A. M. Bekkers, J. F. C. Glatz, J. E. Wildberger, and M. E. Kooi, "MRS: A noninvasive window into cardiac metabolism," NMR Biomed., vol. 28, no. 7, pp. 747-766, 2015.

[69] C. A. Lygate, J. E. Schneider, and S. Neubauer, "Investigating cardiac energetics in heart failure,” Exp Physiol, vol. 98, no. 3, pp. 601-605, 2013.

[70] J. H. Ardenkjaer-Larsen, B. Fridlund, A. Gram, G. Hansson, L. Hansson, M. H. Lerche, R. Servin, M. Thaning, and K. Golman, "Increase in signal-to-noise ratio of $>10,000$ times in liquid-state NMR.,” Proc. Natl. Acad. Sci. U. S. A., vol. 100, no. 18, pp. 10158-63, Sep. 2003.

[71] P. Bhattacharya, E. Y. Chekmenev, W. F. Reynolds, S. Wagner, N. Zacharias, H. R. Chan, R. B??nger, and B. D. Ross, "Parahydrogen-induced polarization (PHIP) hyperpolarized MR receptor imaging in vivo: A pilot study of 13C imaging of atheroma in mice," NMR Biomed., vol. 24, no. 8, pp. 1023-1028, Oct. 2011.

[72] R. A. Green, R. W. Adams, S. B. Duckett, R. E. Mewis, D. C. Williamson, and G. G. R. Green, "The theory and practice of hyperpolarization in magnetic resonance using parahydrogen,” Progress in Nuclear Magnetic Resonance Spectroscopy, vol. 67. pp. 1-48, 2012.

[73] R. V. Shchepin, A. M. Coffey, K. W. Waddell, and E. Y. Chekmenev, "Parahydrogen induced polarization of 1-13C-phospholactate-d 2 for biomedical imaging with $>30,000,000$-fold NMR signal enhancement in water,” Anal. Chem., vol. 86, no. 12, pp. 5601-5605, Jun. 2014.

[74] E. Y. Chekmenev, J. H??vener, V. A. Norton, K. Harris, L. S. Batchelder, P. Bhattacharya, B. D. Ross, and D. P. Weitekamp, "PASADENA hyperpolarization of succinic acid for MRI and NMR spectroscopy,” J. Am. Chem. Soc., vol. 130, no. 13, pp. 4212-4213, 2008.

[75] M. Goez, “Pulse techniques for CIDNP,” Concepts Magn. Reson., vol. 7, no. 4, pp. 263-279, 1995.

[76] P. J. Hore, S. L. Winder, C. H. Roberts, and C. M. Dobson, "Stopped-flow photo-CIDNP observation of protein folding [8],” Journal of the American Chemical Society, vol. 119, no. 21. pp. 5049-5050, 1997.

[77] K. H. Mok and P. J. Hore, "Photo-CIDNP NMR methods for studying protein folding," Methods, vol. 34, no. 1, pp. 75-87, 2004.

[78] X. J. Wang, S. S. Thamarath, A. Alia, B. E. Bode, and J. Matysik, "The solid-state photoCIDNP effect,” Wuli Huaxue Xuebao/ Acta Phys. - Chim. Sin., vol. 32, no. 2, pp. 399-404, 2016.

[79] T. G. Walker and W. Happer, "Spin-exchange optical pumping of noble-gas nuclei,” Rev. Mod. Phys., vol. 69, no. 2, pp. 629-642, 1997.

[80] W. Happer, N. F. 10027 Happer, William (Cotgmbta RaChattort Laboratory, Departmettt of Physics, Cotlmbta Ursioersity, Near Fork, and Optical), “Optical pumping,” Rev. Mod. Phys., 
vol. 44, no. 2, pp. 169-250, 1972.

[81] W. Happer, “Optical pumping,” Rev. Mod. Phys., vol. 44, no. 2, pp. 169-249, 1972.

[82] S. Appelt, A. Baranga, C. Erickson, M. Romalis, A. Young, and W. Happer, "Theory of spinexchange optical pumping of $\wedge\{3\}$ He and $\wedge\{129\}$ Xe,” Phys. Rev. A, vol. 58, no. 2, pp. 14121439, 1998.

[83] A. Korver, D. Thrasher, M. Bulatowicz, and T. G. Walker, "Synchronous Spin-Exchange Optical Pumping,” Phys. Rev. Lett., vol. 115, no. 25, 2015.

[84] D. G. Gadian, K. S. Panesar, A. J. P. Linde, A. J. Horsewill, W. Köckenberger, and J. R. Owers-Bradley, "Preparation of highly polarized nuclear spin systems using brute-force and low-field thermal mixing,” Phys. Chem. Chem. Phys., vol. 14, no. 16, pp. 5397-5402, Apr. 2012.

[85] M. L. Hirsch, N. Kalechofsky, A. Belzer, M. Rosay, and J. G. Kempf, "Brute-Force Hyperpolarization for NMR and MRI,” J. Am. Chem. Soc., vol. 137, no. 26, pp. 8428-8434, 2015.

[86] B. D. Ross, P. Bhattacharya, S. Wagner, T. Tran, and N. Sailasuta, "Hyperpolarized MR imaging: neurologic applications of hyperpolarized metabolism.," AJNR. Am. J. Neuroradiol., vol. 31, no. 1, pp. 24-33, Jan. 2010.

[87] A. Abragam and M. Goldman, "Principles of dynamic nuclear polarisation," Reports Prog. Phys., vol. 41, p. 395, 1978.

[88] M. Borghini, "Spin-Temperature Model of Nuclear Dynamic Polarization Using Free Radicals,” Phys. Rev. Lett., vol. 20, no. 9, pp. 419-421, Feb. 1968.

[89] W. T. Wenckebach, T. J. B. Swanenburg, and N. J. Poulis, "Nuclear dynamic polarization of protons in diluted copper-tutton salts. II,” Physica, vol. 46, no. 2, pp. 303-314, Mar. 1970.

[90] J. H. Ardenkjaer-Larsen, B. Fridlund, A. Gram, G. Hansson, L. Hansson, M. H. Lerche, R. Servin, M. Thaning, and K. Golman, "Increase in signal-to-noise ratio of $>10,000$ times in liquid-state NMR.,” Proc. Natl. Acad. Sci. U. S. A., vol. 100, no. 18, pp. 10158-63, Sep. 2003.

[91] A. S. L. Thankamony, J. J. Wittmann, M. Kaushik, and B. Corzilius, "Dynamic nuclear polarization for sensitivity enhancement in modern solid-state NMR,” Prog. Nucl. Magn. Reson. Spectrosc., vol. 102-103, pp. 120-195, 2017.

[92] W. T. Wenckebach, Essentials of dynamic nuclear polarization. .

[93] A. S. Lilly Thankamony, J. J. Wittmann, M. Kaushik, and B. Corzilius, "Dynamic nuclear polarization for sensitivity enhancement in modern solid-state NMR,” Prog. Nucl. Magn. Reson. Spectrosc., vol. 102-103, pp. 120-195, Nov. 2017.

[94] T. Cheng, M. Mishkovsky, J. A. M. Bastiaansen, O. Ouari, P. Hautle, P. Tordo, B. Van Den Brandt, and A. Comment, "Automated transfer and injection of hyperpolarized molecules with polarization measurement prior to in vivo NMR,” NMR Biomed., vol. 26, no. 11, pp. 15821588, 2013.

[95] J. A. M. Bastiaansen, H. A. I. Yoshihara, Y. Takado, R. Gruetter, and A. Comment, "Hyperpolarized 13C lactate as a substrate for in vivo metabolic studies in skeletal muscle," Metabolomics, pp. 1-9, 2014.

[96] A. Z. Lau, J. J. Miller, M. D. Robson, and D. J. Tyler, "Cardiac perfusion imaging using hyperpolarized 13C urea using flow sensitizing gradients,” Magn. Reson. Med., vol. 75, no. 4, pp. 1474-1483, May 2016.

[97] H. Shang, T. Skloss, C. von Morze, L. Carvajal, M. Van Criekinge, E. Milshteyn, P. E. Z. 
Larson, R. E. Hurd, and D. B. Vigneron, "Handheld electromagnet carrier for transfer of hyperpolarized carbon-13 samples.,” Magn. Reson. Med., Mar. 2015.

[98] J. Milani, B. Vuichoud, A. Bornet, P. Miéville, R. Mottier, S. Jannin, and G. Bodenhausen, “A magnetic tunnel to shelter hyperpolarized fluids,” Rev. Sci. Instrum., vol. 86, no. 2, 2015.

[99] P. J. Randle, "Regulatory interactions between lipids and carbohydrates: the glucose fatty acid cycle after 35 years.,” Diabetes. Metab. Rev., vol. 14, no. 4, pp. 263-83, Dec. 1998.

[100] M. A. Schroeder, H. J. Atherton, M. S. Dodd, P. Lee, L. E. Cochlin, G. K. Radda, K. Clarke, and D. J. Tyler, "The cycling of acetyl-coenzyme A through acetylcarnitine buffers cardiac substrate supply: A hyperpolarized 13C magnetic resonance study,” Circ. Cardiovasc. Imaging, vol. 5, no. 2, pp. 201-209, 2012.

[101] K. X. Moreno, S. M. Sabelhaus, M. E. Merritt, A. D. Sherry, and C. R. Malloy, "Competition of pyruvate with physiological substrates for oxidation by the heart: implications for studies with hyperpolarized [1-13C]pyruvate,” Am J Physiol Hear. Circ Physiol, vol. 298, no. 5, pp. H1556-64, 2010.

[102] M. E. Merritt, C. Harrison, C. Storey, F. M. Jeffrey, A. D. Sherry, and C. R. Malloy, "Hyperpolarized 13C allows a direct measure of flux through a single enzyme-catalyzed step by NMR,” Proc Natl Acad Sci U S A, vol. 104, no. 50, pp. 19773-19777, 2007.

[103] M. A. Schroeder, L. E. Cochlin, L. C. Heather, K. Clarke, G. K. Radda, and D. J. Tyler, "In vivo assessment of pyruvate dehydrogenase flux in the heart using hyperpolarized carbon-13 magnetic resonance,” Proc. Natl. Acad. Sci., vol. 105, no. 33, pp. 12051-12056, Aug. 2008.

[104] H. J. Atherton, M. A. Schroeder, M. S. Dodd, L. C. Heather, E. E. Carter, L. E. Cochlin, S. Nagel, N. R. Sibson, G. K. Radda, K. Clarke, and D. J. Tyler, "Validation of the in vivo assessment of pyruvate dehydrogenase activity using hyperpolarised 13C MRS.," NMR Biomed., vol. 24, no. 2, pp. 201-208, Feb. 2011.

[105] M. A. Schroeder, H. J. Atherton, L. C. Heather, J. L. Griffin, K. Clarke, G. K. Radda, and D. J. Tyler, "Determining the in vivo regulation of cardiac pyruvate dehydrogenase based on label flux from hyperpolarised [1-13C]pyruvate,” NMR Biomed., vol. 24, no. 8, pp. 980-987, Oct. 2011.

[106] M. H. Lauritzen, C. Laustsen, S. A. Butt, P. Magnusson, L. V. Søgaard, J. H. ArdenkjærLarsen, and P. Åkeson, "Enhancing the [13C]bicarbonate signal in cardiac hyperpolarized [113C]pyruvate MRS studies by infusion of glucose, insulin and potassium,” NMR Biomed., vol. 26, no. 11, pp. 1496-1500, 2013.

[107] E. S. S. Hansen, R. S. Tougaard, T. S. Nørlinger, E. Mikkelsen, P. M. Nielsen, L. B. Bertelsen, H. E. Bøtker, H. S. Jørgensen, and C. Laustsen, "Imaging porcine cardiac substrate selection modulations by glucose, insulin and potassium intervention: A hyperpolarized [113C]pyruvate study,” NMR Biomed., vol. 30, no. 6, 2017.

[108] C. Khemtong, N. R. Carpenter, L. L. Lumata, M. E. Merritt, K. X. Moreno, Z. Kovacs, C. R. Malloy, and A. D. Sherry, "Hyperpolarized13C NMR detects rapid drug-induced changes in cardiac metabolism,” Magn. Reson. Med., vol. 74, no. 2, pp. 312-319, 2015.

[109] R. S. Tougaard, E. S. S. Hansen, C. Laustsen, J. Lindhardt, M. Schroeder, H. E. Bøtker, W. Y. Kim, H. Wiggers, and H. Stødkilde-Jørgensen, "Acute hypertensive stress imaged by cardiac hyperpolarized $\left[1-{ }^{13}\right.$ C]pyruvate magnetic resonance,” Magn. Reson. Med., no. September 2017, pp. 1-9, 2018.

[110] M. S. Dodd, V. Ball, R. Bray, H. Ashrafian, H. Watkins, K. Clarke, and D. J. Tyler, "In vivo mouse cardiac hyperpolarized magnetic resonance spectroscopy,” J. Cardiovasc. Magn. Reson., vol. 15, no. 1, p. 19, Feb. 2013. 
[111] A. J. Bakermans, M. S. Dodd, K. Nicolay, J. J. Prompers, D. J. Tyler, and S. M. Houten, "Myocardial energy shortage and unmet anaplerotic needs in the fasted long-chain acyl-CoA dehydrogenase knockout mouse,” Cardiovasc. Res., vol. 100, no. 3, pp. 441-449, Dec. 2013.

[112] M. A. Schroeder, H. J. Atherton, D. R. Ball, M. A. Cole, L. C. Heather, J. L. Griffin, K. Clarke, G. K. Radda, and D. J. Tyler, "Real-time assessment of Krebs cycle metabolism using hyperpolarized 13C magnetic resonance spectroscopy.,” FASEB J., vol. 23, no. 8, pp. 252938, Aug. 2009.

[113] H. Scholte, R. Rodrigues Pereira, P. de Jonge, I. Luyt-Houwen, M. Hedwig, M. Verduin, and J. Ross, "Primary carnitine deficiency.," J. Clin. ..., vol. 28, no. 5, pp. 351-7, 1990.

[114] H. J. Atherton, M. S. Dodd, L. C. Heather, M. A. Schroeder, J. L. Griffin, G. K. Radda, K. Clarke, and D. J. Tyler, "Role of pyruvate dehydrogenase inhibition in the development of hypertrophy in the hyperthyroid rat heart: A combined magnetic resonance imaging and hyperpolarized magnetic resonance spectroscopy study,” Circulation, vol. 123, no. 22, pp. 2552-2561, 2011.

[115] A. P. Chen, R. E. Hurd, M. A. Schroeder, A. Z. Lau, Y. P. Gu, W. W. Lam, J. Barry, J. Tropp, and C. H. Cunningham, "Simultaneous investigation of cardiac pyruvate dehydrogenase flux, Krebs cycle metabolism and $\mathrm{pH}$, using hyperpolarized [1,2- 13C 2]pyruvate in vivo,” NMR Biomed., vol. 25, no. 2, pp. 305-311, 2012.

[116] A. P. Chen, A. Z. Lau, Y. Gu, M. e A. Schroeder, J. Barry, and C. H. Cunningham, "Probing the cardiac malate-aspartate shuttle non-invasively using hyperpolarized [1,2-13C2]pyruvate," NMR Biomed., 2017.

[117] A. P. Chen, J. Kurhanewicz, R. Bok, D. Xu, D. Joun, V. Zhang, S. J. Nelson, R. E. Hurd, and D. B. Vigneron, "Feasibility of using hyperpolarized [1-13C]lactate as a substrate for in vivo metabolic 13C MRSI studies,” Magn. Reson. Imaging, vol. 26, no. 6, pp. 721-726, 2008.

[118] D. Mayer, Y.-F. Yen, S. Josan, J. M. Park, A. Pfefferbaum, R. E. Hurd, and D. M. Spielman, "Application of hyperpolarized [1-(13) C]lactate for the in vivo investigation of cardiac metabolism.,” NMR Biomed., vol. 25, no. 10, pp. 1119-24, 2012.

[119] A. P. Chen, J. Y. C. Lau, R. D. A. Alvares, and C. H. Cunningham, "Using [1-13C]lactic acid for hyperpolarized 13C MR cardiac studies,” Magn. Reson. Med., vol. 73, no. June, pp. 20872093, 2015.

[120] S. Hu, M. Zhu, H. A. I. Yoshihara, D. M. Wilson, K. R. Keshari, P. Shin, G. Reed, C. von Morze, R. Bok, P. E. Z. Larson, J. Kurhanewicz, and D. B. Vigneron, "In vivo measurement of normal rat intracellular pyruvate and lactate levels after injection of hyperpolarized [113C]alanine,” Magn. Reson. Imaging, vol. 29, no. 8, pp. 1035-1040, 2011.

[121] J. a. M. Bastiaansen, T. Cheng, H. Lei, R. Gruetter, and A. Comment, "Direct noninvasive estimation of myocardial tricarboxylic acid cycle flux in vivo using hyperpolarized 13C magnetic resonance,” J. Mol. Cell. Cardiol., vol. 87, pp. 129-137, 2015.

[122] U. Koellisch, C. V. Gringeri, G. Rancan, E. V. Farell, M. I. Menzel, A. Haase, M. Schwaiger, and R. F. Schulte, "Metabolic imaging of hyperpolarized $\left[1-{ }^{13} \mathrm{C}\right]$ acetate and [1$\left.{ }^{13} \mathrm{C}\right]$ acetylcarnitine - Investigation of the influence of dobutamine induced stress," Magn. Reson. Med., vol. 74, no. 4, pp. 1011-1018, 2015.

[123] D. R. Ball, B. Rowlands, M. S. Dodd, L. Le Page, V. Ball, C. A. Carr, K. Clarke, and D. J. Tyler, "Hyperpolarized butyrate: A metabolic probe of short chain fatty acid metabolism in the heart,” Magn. Reson. Med., vol. 71, no. 5, pp. 1663-1669, 2014.

[124] J. A. M. Bastiaansen, M. E. Merritt, and A. Comment, "Measuring changes in substrate utilization in the myocardium in response to fasting using hyperpolarized [1-13C]butyrate and 
[1-13C]pyruvate,” Sci. Rep., vol. 6, no. 1, p. 25573, 2016.

[125] W. C. Chen, H. Q. Teo, and P. Lee, "Investigating in vivo cardiac ketone body metabolism using hyperpolarized 13C acetoacetate," in Proceeding of the International Society for Magnetic Resonance in Medicine, 2016, p. 474.

[126] W. Chen, C. Khemtong, W. Jiang, C. R. Malloy, and A. . Sherry, "Metabolism of hyperpolarized 13C-acetoacetate/B-hydroxybutyrate reveals mitochondrial redox state in perfused rat hearts.,” in International Society for Magnetic Resonance in Medicine, 2016, p. 672.

[127] J. J. Miller, D. R. Ball, A. Z. Lau, and D. J. Tyler, "Hyperpolarised Ketone Body Metabolism in the Rat Heart,” NMR Biomed., p. in press, 2018.

[128] L. C. Heather, K. M. Pates, H. J. Atherton, M. A. Cole, D. R. Ball, R. D. Evans, J. F. Glatz, J. J. Luiken, J. L. Griffin, and K. Clarke, "Differential translocation of the fatty acid transporter, FAT/CD36, and the glucose transporter, GLUT4, coordinates changes in cardiac substrate metabolism during ischemia and reperfusion,” Circ Hear. Fail, vol. 6, no. 5, pp. 1058-1066, 2013.

[129] M. E. Merritt, C. Harrison, C. Storey, A. D. Sherry, and C. R. Malloy, "Inhibition of carbohydrate oxidation during the first minute of reperfusion after brief ischemia: NMR detection of hyperpolarized $13 \mathrm{CO} 2$ and H13CO3," Magn Reson Med, vol. 60, no. 5, pp. 10291036, 2008.

[130] D. R. Ball, R. Cruickshank, C. A. Carr, D. J. Stuckey, P. Lee, K. Clarke, and D. J. Tyler, "Metabolic imaging of acute and chronic infarction in the perfused rat heart using hyperpolarised [1-13C]pyruvate,” NMR Biomed., vol. 26, no. 11, pp. 1441-1450, 2013.

[131] D. Oh-Ici, P. Wespi, J. Busch, L. Wissmann, M. Krajewski, K. Weiss, A. Sigfridsson, D. Messroghli, and S. Kozerke, "Hyperpolarized Metabolic MR Imaging of Acute Myocardial Changes and Recovery after Ischemia-Reperfusion in a Small-Animal Model,” Radiology, vol. 278, no. 3, pp. 742-751, 2016.

[132] H. A. Yoshihara, J. A. Bastiaansen, C. Berthonneche, A. Comment, and J. Schwitter, “An intact small animal model of myocardial ischemia-reperfusion: Characterization of metabolic changes by hyperpolarized 13C MR spectroscopy,” Am J Physiol Hear. Circ Physiol, vol. 309, no. 12, pp. H2058-66, 2015.

[133] M. S. Dodd, H. J. Atherton, C. A. Carr, D. J. Stuckey, J. A. West, J. L. Griffin, G. K. Radda, K. Clarke, L. C. Heather, and D. J. Tyler, "Impaired in vivo mitochondrial krebs cycle activity after myocardial infarction assessed using hyperpolarized magnetic resonance spectroscopy,” Circ. Cardiovasc. Imaging, vol. 7, no. 6, pp. 895-904, 2014.

[134] L. J. Shaw, D. S. Berman, D. J. Maron, G. B. J. Mancini, S. W. Hayes, P. M. Hartigan, W. S. Weintraub, R. A. O’Rourke, M. Dada, J. A. Spertus, B. R. Chaitman, J. Friedman, P. Slomka, G. V. Heller, G. Germano, G. Gosselin, P. Berger, W. J. Kostuk, R. G. Schwartz, M. Knudtson, E. Veledar, E. R. Bates, B. McCallister, K. K. Teo, and W. E. Boden, “Optimal medical therapy with or without percutaneous coronary intervention to reduce ischemic burden: results from the Clinical Outcomes Utilizing Revascularization and Aggressive Drug Evaluation (COURAGE) trial nuclear substudy,” Circulation, vol. 117, no. 10, pp. 1283-1291, 2008.

[135] C. H. Orchard and H. E. Cingolani, “Acidosis and arrhythmias in cardiac muscle,” Cardiovasc. Res., vol. 28, no. 9, pp. 1312-1319, 1994.

[136] M. A. Schroeder, P. Swietach, H. J. Atherton, F. A. Gallagher, P. Lee, G. K. Radda, K. Clarke, and D. J. Tyler, "Measuring intracellular $\mathrm{pH}$ in the heart using hyperpolarized carbon dioxide and bicarbonate: A 13C and 31P magnetic resonance spectroscopy study," Cardiovasc. Res., 
vol. 86, no. 1, pp. 82-91, 2010.

[137] F. A. Gallagher, M. I. Kettunen, and K. M. Brindle, "Imaging pH with hyperpolarized 13C,” NMR in Biomedicine, vol. 24, no. 8. pp. 1006-1015, 2011.

[138] A. Z. Lau, J. J. Miller, and D. J. Tyler, "Mapping of intracellular pH in the in vivo rodent heart using hyperpolarized [1-13C]pyruvate,” Magn. Reson. Med., vol. 77, no. 5, pp. 1810-1817, May 2017.

[139] A. Z. Lau, J. J. Miller, M. D. Robson, and D. J. Tyler, "Simultaneous assessment of cardiac metabolism and perfusion using copolarized [1-13C]pyruvate and 13C-urea,” Magn. Reson. Med., vol. 77, no. 1, pp. 151-158, 2017.

[140] K. Golman, J. S. Petersson, P. Magnusson, E. Johansson, P. Åkeson, C.-M. Chai, G. Hansson, and S. Månsson, "Cardiac metabolism measured noninvasively by hyperpolarized13C MRI," Magn. Reson. Med., vol. 59, no. 5, pp. 1005-1013, May 2008.

[141] A. J. Lewis, J. J. Miller, A. Z. Lau, M. K. Curtis, O. J. Rider, R. P. Choudhury, S. Neubauer, C. H. Cunningham, C. A. Carr, and D. J. Tyler, "Non-Invasive Immuno-Metabolic Cardiac Inflammation Imaging Using Hyperpolarized Magnetic Resonance,” Circ. Res., p. CIRCRESAHA.117.312535, Feb. 2018.

[142] J. J. Miller, A. Z. Lau, P. M. Nielsen, G. McMullen-Klein, A. J. Lewis, N. R. Jespersen, V. Ball, F. A. Gallagher, C. A. Carr, C. Laustsen, H. E. Bøtker, D. J. Tyler, and M. A. Schroeder, "Hyperpolarized [1,4-13C2]Fumarate Enables Magnetic Resonance-Based Imaging of Myocardial Necrosis,” JACC: Cardiovascular Imaging, 2017.

[143] F. A. Gallagher, M. I. Kettunen, D.-E. Hu, P. R. Jensen, R. i. t Zandt, M. Karlsson, A. Gisselsson, S. K. Nelson, T. H. Witney, S. E. Bohndiek, G. Hansson, T. Peitersen, M. H. Lerche, and K. M. Brindle, "Production of hyperpolarized [1,4-13C2]malate from [1,413C2]fumarate is a marker of cell necrosis and treatment response in tumors," Proc. Natl. Acad. Sci., vol. 106, no. 47, pp. 19801-19806, 2009.

[144] M. S. Dodd, D. R. Ball, M. A. Schroeder, L. M. Le Page, H. J. Atherton, L. C. Heather, A. M. Seymour, H. Ashrafian, H. Watkins, K. Clarke, and D. J. Tyler, "In vivo alterations in cardiac metabolism and function in the spontaneously hypertensive rat heart," Cardiovasc. Res., vol. 95, no. 1, pp. 69-76, 2012.

[145] B. J. Maron and M. S. Maron, “Hypertrophic cardiomyopathy,” Lancet, vol. 381, no. 9862, pp. 242-255, 2013.

[146] A.-M. L. Seymour, L. Giles, V. Ball, J. J. Miller, K. Clarke, C. A. Carr, and D. J. Tyler, “In vivo assessment of cardiac metabolism and function in the abdominal aortic banding model of compensated cardiac hypertrophy.," Cardiovasc. Res., vol. 106, no. 2, pp. 249-260, 2015.

[147] M. A. Schroeder, A. Z. Lau, A. P. Chen, Y. Gu, J. Nagendran, J. Barry, X. Hu, J. R. B. Dyck, D. J. Tyler, K. Clarke, K. A. Connelly, G. A. Wright, and C. H. Cunningham, "Hyperpolarized 13C magnetic resonance reveals early- and late-onset changes to in vivo pyruvate metabolism in the failing heart,” Eur. J. Heart Fail., vol. 15, no. 2, pp. 130-140, 2013.

[148] S. Boudina and E. D. Abel, “Diabetic cardiomyopathy revisited,” Circulation, vol. 115, no. 25. pp. 3213-3223, 2007.

[149] L. M. Le Page, O. J. Rider, A. J. Lewis, V. Ball, K. Clarke, E. Johansson, C. A. Carr, L. C. Heather, and D. J. Tyler, "Increasing pyruvate dehydrogenase flux as a treatment for diabetic cardiomyopathy: A combined 13C hyperpolarized magnetic resonance and echocardiography study,” Diabetes, vol. 64, no. 8, pp. 2735-2743, 2015.

[150] A. J. Lewis, J. J. Miller, C. McCallum, O. J. Rider, S. Neubauer, L. C. Heather, and D. J. Tyler, “Assessment of Metformin Induced Changes in Cardiac and Hepatic Redox State Using 
Hyperpolarized[1-13C]Pyruvate.,” Diabetes, p. db160804, 2016.

[151] A. A. Tahrani, G. I. Varughese, J. H. Scarpello, and F. W. F. Hanna, "Metformin, heart failure, and lactic acidosis: is metformin absolutely contraindicated?," $B M J$, vol. 335, no. 7618, pp. 508-12, 2007.

[152] D. Savic, K. Timm, V. Ball, L. Heather, and D. Tyler, "In Vivo Hyperpolarized MRS Study Showing Improved Cardiac Metabolism in Type 1 Diabetes with Daily L-Carnitine Treatment,” 2017, p. 0542.

[153] D. Martin, H. A. I. Yoshihara, E. Can, R. Hullin, and J. A. M. Bastiaansen, "Measurement of metabolic changes in acute doxorubicin-induced cardiotoxicity in mice using hyperpolarized [1-13C]pyruvate,” in International Society for Magnetic Resonance in Medicine, 2017, p. 0170 .

[154] K. N. Timm, J. J. Miller, D. Savic, V. Ball, L. Giles, C.-R. Chong, M. S. Dodd, and D. J. Tyler, "Metabolic changes in the heart precede functional changes in a rat model of doxorubicin-induced cardiotoxicity," in International Society for Magnetic Resonance in Medicine, 2017, p. 3605.

[155] O. J. Rider and D. J. Tyler, "Clinical implications of cardiac hyperpolarized magnetic resonance imaging,” J Cardiovasc Magn Reson, vol. 15, p. 93, 2013.

[156] M. A. Schroeder, K. Clarke, S. Neubauer, and D. J. Tyler, "Hyperpolarized magnetic resonance: a novel technique for the in vivo assessment of cardiovascular disease," Circulation, vol. 124, no. 14, pp. 1580-1594, 2011.

[157] P. S. Biesbroek, A. M. Beek, T. Germans, H. W. M. Niessen, and A. C. Van Rossum, "Diagnosis of myocarditis: Current state and future perspectives," Int. J. Cardiol., vol. 191, pp. 211-219, 2015.

[158] L. Guilherme and J. Kalil, "Rheumatic fever and rheumatic heart disease: Cellular mechanisms leading autoimmune reactivity and disease,” Journal of Clinical Immunology, vol. 30, no. 1. pp. 17-23, 2010.

[159] A. J. Lewis, J. J. Miller, A. Z. Lau, M. K. Curtis, O. J. Rider, R. P. Choudhury, S. Neubauer, C. H. Cunningham, C. A. Carr, and D. J. Tyler, "Non-Invasive Immuno-Metabolic Cardiac Inflammation Imaging Using Hyperpolarized Magnetic Resonance,” Circ. Res., p. CIRCRESAHA.117.312535, Feb. 2018.

[160] M. C. Cimolai, S. Alvarez, C. Bode, and H. Bugger, "Mitochondrial mechanisms in septic cardiomyopathy,” International Journal of Molecular Sciences, vol. 16, no. 8. pp. 1776317778, 2015.

[161] S. J. Nelson, J. Kurhanewicz, D. B. Vigneron, P. E. Z. Larson, A. L. Harzstark, M. Ferrone, M. van Criekinge, J. W. Chang, R. Bok, I. Park, G. Reed, L. Carvajal, E. J. Small, P. Munster, V. K. Weinberg, J. H. Ardenkjaer-Larsen, A. P. Chen, R. E. Hurd, L.-I. Odegardstuen, F. J. Robb, J. Tropp, and J. A. Murray, "Metabolic imaging of patients with prostate cancer using hyperpolarized [1-13 C]pyruvate.,” Sci. Transl. Med., vol. 5, no. 198, p. 198ra108, 2013.

[162] R. Aggarwal, D. B. Vigneron, and J. Kurhanewicz, “Hyperpolarized 1-[13C]-Pyruvate Magnetic Resonance Imaging Detects an Early Metabolic Response to Androgen Ablation Therapy in Prostate Cancer,” European Urology, 2017.

[163] D. Tyler, O. Rider, M. Dodd, A. Lau, A. Lewis, J. Miller, M. Peterzan, C. Trumper, and S. Neubauer, "Demonstrating the Randle Cycle in Vivo: Assessment of Physiological Alterations in Human Cardiac Metabolism Using Hyperpolarised 13C MR Spectroscopy,” in International Society for Magnetic Resonance in Medicine, 2017, p. 0726.

[164] C. H. Cunningham, J. Y. C. Lau, A. P. Chen, B. J. Geraghty, W. J. Perks, I. Roifman, G. A. 
Wright, and K. A. Connelly, "Hyperpolarized 13C Metabolic MRI of the Human Heart: Initial Experience,” Circ. Res., vol. 119, no. 11, pp. 1177-1182, 2016.

[165] J. H. Ardenkjaer-Larsen, A. M. Leach, N. Clarke, J. Urbahn, D. Anderson, and T. W. Skloss, "Dynamic nuclear polarization polarizer for sterile use intent," NMR Biomed., vol. 24, no. 8, pp. 927-932, 2011.

[166] A. Z. Lau, A. P. Chen, and C. H. Cunningham, "Integrated Bloch-Siegert B 1 mapping and multislice imaging of hyperpolarized 13C pyruvate and bicarbonate in the heart," Magn. Reson. Med., vol. 67, no. 1, pp. 62-71, Jan. 2012.

[167] J. Maidens and M. Arcak, "Semidefinite relaxations in optimal experiment design with application to substrate injection for hyperpolarized MRI,” p. 6, Oct. 2015.

[168] G. Giovannetti, V. Hartwig, F. Frijia, L. Menichetti, V. Positano, J. H. Ardenkjaer-Larsen, V. Lionetti, G. D. Aquaro, D. De Marchi, A. Flori, L. Landini, M. Lombardi, and M. F. Santarelli, "Hyperpolarized 13C MRS Cardiac Metabolism Studies in Pigs: Comparison Between Surface and Volume Radiofrequency Coils,” Appl. Magn. Reson., vol. 42, no. 3, pp. 413-428, Apr. 2012.

[169] G. Giovannetti, F. Frijia, L. Menichetti, M. Milanesi, J. H. Ardenkjaer-Larsen, D. De Marchi, V. Hartwig, V. Positano, L. Landini, M. Lombardi, and M. F. Santarelli, "Hyperpolarized 13C MRS surface coil: design and signal-to-noise ratio estimation.,” Med. Phys., vol. 37, no. 10, pp. 5361-5369, 2010.

[170] J. D. Sanchez-Heredia, E. S. Szocska Hansen, C. Laustsen, V. Zhurbenko, and J. H. Ardenkjær-Larsen, "Low-Noise Active Decoupling Circuit and its Application to 13C Cryogenic RF Coils at 3T,” Tomography, vol. 3, no. 1, p. 60:66, 2017.

[171] J. Maidens, J. W. Gordon, M. Arcak, and P. E. Z. Larson, “Optimizing Flip Angles for Metabolic Rate Estimation in Hyperpolarized Carbon-13 MRI,” IEEE Trans. Med. Imaging, vol. 35, no. 11, pp. 2403-2412, Nov. 2016.

[172] J. Maidens and M. Arcak, "Semidefinite relaxations in optimal experiment design with application to substrate injection for hyperpolarized MRI,” p. 6, Oct. 2015.

[173] K. Golman, R. in 't Zandt, and M. Thaning, "Real-time metabolic imaging.," Proc. Natl. Acad. Sci. U. S. A., vol. 103, no. 30, pp. 11270-5, Jul. 2006.

[174] S. E. Day, M. I. Kettunen, F. A. Gallagher, D.-E. Hu, M. Lerche, J. Wolber, K. Golman, J. H. Ardenkjaer-Larsen, and K. M. Brindle, "Detecting tumor response to treatment using hyperpolarized 13C magnetic resonance imaging and spectroscopy.,” Nat. Med., vol. 13, no. 11, pp. 1382-7, Nov. 2007.

[175] Y. Xing, G. D. Reed, J. M. Pauly, A. B. Kerr, and P. E. Z. Z. Larson, “Optimal variable flip angle schemes for dynamic acquisition of exchanging hyperpolarized substrates.,” J. Magn. Reson., vol. 234, pp. 75-81, Sep. 2013.

[176] K. Nagashima, "Optimum pulse flip angles for multi-scan acquisition of hyperpolarized NMR and MRI,” J. Magn. Reson., vol. 190, no. 2, pp. 183-188, 2008.

[177] L. Menichetti, F. Frijia, A. Flori, F. Wiesinger, V. Lionetti, G. Giovannetti, G. D. Aquaro, F. A. Recchia, J. H. Ardenkjaer-Larsen, M. F. Santarelli, and M. Lombardi, “Assessment of realtime myocardial uptake and enzymatic conversion of hyperpolarized [1-13C]pyruvate in pigs using slice selective magnetic resonance spectroscopy," Contrast Media Mol. Imaging, vol. 7, no. 1, pp. 85-94, Jan. 2012.

[178] L. M. Le Page, D. R. Ball, V. Ball, M. S. Dodd, J. J. Miller, L. C. Heather, and D. J. Tyler, "Simultaneous in vivo assessment of cardiac and hepatic metabolism in the diabetic rat using hyperpolarized MRS,” NMR Biomed., vol. 29, no. 12, pp. 1759-1767, 2016. 
[179] M. Marjańska, I. Iltis, A. A. Shestov, D. K. Deelchand, C. Nelson, K. Uurbil, P.-G. G. Henry, K. Uğurbil, and P.-G. G. Henry, "In vivo 13C spectroscopy in the rat brain using hyperpolarized [1-13C]pyruvate and [2-13C]pyruvate,” J. Magn. Reson., vol. 206, no. 2, pp. 210-218, 2010.

[180] C. H. Cunningham, A. P. Chen, M. J. Albers, J. Kurhanewicz, R. E. Hurd, Y. F. Yen, J. M. Pauly, S. J. Nelson, and D. B. Vigneron, "Double spin-echo sequence for rapid spectroscopic imaging of hyperpolarized 13C,” J. Magn. Reson., vol. 187, no. 2, pp. 357-362, Aug. 2007.

[181] A. P. Chen, K. Leung, W. Lam, R. E. Hurd, D. B. Vigneron, and C. H. Cunningham, “Design of spectral-spatial outer volume suppression RF pulses for tissue specific metabolic characterization with hyperpolarized 13C pyruvate,” J. Magn. Reson., vol. 200, no. 2, pp. 344348, Oct. 2009.

[182] S. E. Day, M. I. Kettunen, M. K. Cherukuri, J. B. Mitchell, M. J. Lizak, H. D. Morris, S. Matsumoto, A. P. Koretsky, and K. M. Brindle, "Detecting response of rat C6 glioma tumors to radiotherapy using hyperpolarized [1-13C]pyruvate and 13C magnetic resonance spectroscopic imaging,” Magn. Reson. Med., vol. 65, no. 2, pp. 557-563, Feb. 2011.

[183] K. Golman and J. S. Petersson, "Metabolic imaging and other applications of hyperpolarized 13C1.," Acad. Radiol., vol. 13, no. 8, pp. 932-942, Aug. 2006.

[184] Y. F. Yen, S. J. Kohler, A. P. Chen, J. Tropp, R. Bok, J. Wolber, M. J. Albers, K. A. Gram, M. L. Zierhut, I. Park, V. Zhang, S. Hu, S. J. Nelson, D. B. Vigneron, J. Kurhanewicz, H. A. A. M. Dirven, and R. E. Hurd, "Imaging considerations for in vivo 13C metabolic mapping using hyperpolarized 13C-pyruvate,” Magn. Reson. Med., vol. 62, no. 1, pp. 1-10, Jul. 2009.

[185] M. M. Darpolor, Y. F. Yen, M. S. Chua, L. Xing, R. H. Clarke-Katzenberg, W. Shi, D. Mayer, S. Josan, R. E. Hurd, A. Pfefferbaum, L. Senadheera, S. So, L. V. Hofmann, G. M. Glazer, and D. M. Spielman, "In vivo MRSI of hyperpolarized [1-13C]pyruvate metabolism in rat hepatocellular carcinoma,” NMR Biomed., vol. 24, no. 5, pp. 506-513, Jun. 2011.

[186] A. E. Hansen, F. L. Andersen, S. T. Henriksen, A. Vignaud, J. H. Ardenkjaer-Larsen, L. Højgaard, A. Kjaer, and T. L. Klausen, "Simultaneous PET/MRI with (13)C magnetic resonance spectroscopic imaging (hyperPET): phantom-based evaluation of PET quantification.,” EJNMMI Phys., vol. 3, no. 1, 2016.

[187] D. D. D. Belkić and K. Belkić, "The fast Padé transform in magnetic resonance spectroscopy for potential improvements in early cancer diagnostics.," Phys. Med. Biol., vol. 50, no. 18, pp. 4385-4408, Sep. 2005.

[188] D. Mayer, Y. F. Yen, Y. S. Levin, J. Tropp, A. Pfefferbaum, R. E. Hurd, and D. M. Spielman, "In vivo application of sub-second spiral chemical shift imaging (CSI) to hyperpolarized 13C metabolic imaging: Comparison with phase-encoded CSI,” J. Magn. Reson., vol. 204, no. 2, pp. 340-345, Jun. 2010.

[189] J.-X. Wang, M. E. Merritt, A. D. Sherry, and C. R. Malloy, “Accelerated chemical shift imaging of hyperpolarized 13C metabolites,” Magn. Reson. Med., Jul. 2016.

[190] S. Hu, M. Lustig, A. Balakrishnan, P. E. Z. Larson, R. Bok, J. Kurhanewicz, S. J. Nelson, A. Goga, J. M. Pauly, and D. B. Vigneron, “3D compressed sensing for highly accelerated hyperpolarized 13C MRSI with in vivo applications to transgenic mouse models of cancer," Magn. Reson. Med., vol. 63, no. 2, pp. 312-321, Feb. 2010.

[191] M. Durst, U. Koellisch, A. Frank, G. Rancan, C. V. Gringeri, V. Karas, F. Wiesinger, M. I. Menzel, M. Schwaiger, A. Haase, and R. F. Schulte, "Comparison of acquisition schemes for hyperpolarised 13C imaging,” NMR Biomed., vol. 28, no. 6, pp. 715-725, Jun. 2015.

[192] F. Wiesinger, E. Weidl, M. I. Menzel, M. A. Janich, O. Khegai, S. J. Glaser, A. Haase, M. 
Schwaiger, and R. F. Schulte, "IDEAL spiral CSI for dynamic metabolic MR imaging of hyperpolarized [1-13C]pyruvate.,” Magn. Reson. Med., vol. 68, no. 1, pp. 8-16, Jul. 2012.

[193] O. Khegai, R. F. Schulte, M. A. Janich, M. I. Menzel, E. Farrell, A. M. Otto, J. H. ArdenkjaerLarsen, S. J. Glaser, A. Haase, M. Schwaiger, and F. Wiesinger, “Apparent rate constant mapping using hyperpolarized [1-(13)C]pyruvate.,” NMR Biomed., vol. 27, no. 10, pp. 125665, Oct. 2014.

[194] U. Koellisch, C. V. Gringeri, G. Rancan, E. V. Farell, M. I. Menzel, A. Haase, M. Schwaiger, and R. F. Schulte, "Metabolic imaging of hyperpolarized [1-13C]acetate and [1-

13C]acetylcarnitine - Investigation of the influence of dobutamine induced stress,” Magn. Reson. Med., vol. 74, no. 4, pp. 1011-1018, Oct. 2015.

[195] L. Menichetti, F. Frijia, A. Flori, V. Lionetti, M. Liserani, G. Giovannetti, G. Bianchi, S. L. Romano, V. Positano, J. H. Ardenkjaer-larsen, R. F. Schulte, F. A. Recchia, L. Landini, M. F. Santarelli, and M. Lombardi, "3D cardiac Chemical Shift Imaging of [ 1-13C ] hyperpolarized acetate and pyruvate in pigs,” in Journal of Cardiovascular Magnetic Resonance, 2013, vol. 15, no. Suppl 1, p. P10.

[196] J. J. Miller, A. Z. Lau, I. Teh, J. E. J. E. J. E. Schneider, P. Kinchesh, S. Smart, V. Ball, N. R. Sibson, and D. J. Tyler, "Robust and high resolution hyperpolarized metabolic imaging of the rat heart at 7T with 3D spectral-spatial EPI,” Magn. Reson. Med., vol. 75, no. 4, pp. 15151524, May 2015.

[197] A. Z. Lau, A. P. Chen, R. E. Hurd, and C. H. Cunningham, "Spectral-spatial excitation for rapid imaging of DNP compounds.,” NMR Biomed., vol. 24, no. 8, pp. 988-96, Oct. 2011.

[198] R. F. Schulte, J. I. Sperl, E. Weidl, M. I. Menzel, M. A. Janich, O. Khegai, M. Durst, J. H. Ardenkjaer-Larsen, S. J. Glaser, A. Haase, M. Schwaiger, and F. Wiesinger, "Saturationrecovery metabolic-exchange rate imaging with hyperpolarized [1-(13) C] pyruvate using spectral-spatial excitation.," Magn. Reson. Med., vol. 69, no. 5, pp. 1209-16, May 2012.

[199] C. H. Cunningham, A. P. Chen, M. Lustig, B. A. Hargreaves, J. Lupo, D. Xu, J. Kurhanewicz, R. E. Hurd, J. M. Pauly, S. J. Nelson, and D. B. Vigneron, "Pulse sequence for dynamic volumetric imaging of hyperpolarized metabolic products.,” J. Magn. Reson., vol. 193, no. 1, pp. 139-46, Jul. 2008.

[200] P. Bhattacharya, E. Y. Chekmenev, W. H. Perman, K. C. Harris, A. P. Lin, V. A. Norton, C. T. Tan, B. D. Ross, and D. P. Weitekamp, "Towards hyperpolarized 13C-succinate imaging of brain cancer,” 2007.

[201] A. Sigfridsson, K. Weiss, L. Wissmann, J. Busch, M. Krajewski, M. Batel, G. Batsios, M. Ernst, and S. Kozerke, "Hybrid multiband excitation multiecho acquisition for hyperpolarized (13) C spectroscopic imaging.,” Magn. Reson. Med., May 2014.

[202] A. P. Chen, K. Leung, W. Lam, R. E. Hurd, D. B. Vigneron, and C. H. Cunningham, "Design of spectral-spatial outer volume suppression RF pulses for tissue specific metabolic characterization with hyperpolarized 13C pyruvate,” J. Magn. Reson., vol. 200, no. 2, pp. 344348, Oct. 2009.

[203] P. E. Z. Z. Larson, A. B. Kerr, A. P. Chen, M. S. Lustig, M. L. Zierhut, S. Hu, C. H. Cunningham, J. M. Pauly, J. Kurhanewicz, and D. B. Vigneron, "Multiband excitation pulses for hyperpolarized 13C dynamic chemical-shift imaging,” J. Magn. Reson., vol. 194, no. 1, pp. 121-127, Sep. 2008.

[204] C. H. Cunningham, J. Y. C. C. Lau, A. P. Chen, B. J. Geraghty, W. J. Perks, I. Roifman, G. A. Wright, and K. A. Connelly, "Hyperpolarized 13 C Metabolic MRI of the Human Heart: Initial Experience,” Circ. Res., vol. 119, no. 11, pp. 1177-1182, Nov. 2016. 
[205] C. H. Cunningham, W. Dominguez Viqueira, R. E. Hurd, and A. P. Chen, "Frequency correction method for improved spatial correlation of hyperpolarized 13C metabolites and anatomy.,” NMR Biomed., vol. 27, no. 2, pp. 212-8, Feb. 2014.

[206] J. J. Miller, A. Z. Lau, and D. J. Tyler, "Susceptibility-induced distortion correction in hyperpolarized echo planar imaging,” Magn. Reson. Med., 2017.

[207] B. J. Geraghty, J. Y. C. Lau, A. P. Chen, and C. H. Cunningham, "Dual-Echo EPI sequence for integrated distortion correction in 3D time-resolved hyperpolarized 13C MRI," Magnetic Resonance in Medicine, vol. 79, no. 2, pp. 643-653, 01-Apr-2017.

[208] J. Pfeuffer, P.-F. Van de Moortele, K. Ugurbil, X. Hu, and G. H. Glover, "Correction of physiologically induced global off-resonance effects in dynamic echo-planar and spiral functional imaging.,” Magn. Reson. Med., vol. 47, no. 2, pp. 344-53, Feb. 2002.

[209] D. Mayer, Y.-F. F. Yen, J. Tropp, A. Pfefferbaum, R. E. Hurd, and D. M. Spielman, "Application of subsecond spiral chemical shift imaging to real-time multislice metabolic imaging of the rat in vivo after injection of hyperpolarized 13C1-pyruvate.," Magn. Reson. Med., vol. 62, no. 3, pp. 557-564, Sep. 2009.

[210] A. Sigfridsson, K. Weiss, L. Wissmann, J. Busch, M. Krajewski, M. Batel, G. Batsios, M. Ernst, and S. Kozerke, "Hybrid multiband excitation multiecho acquisition for hyperpolarized (13) C spectroscopic imaging.," Magn. Reson. Med., May 2014.

[211] J. Wang, A. J. Wright, D.-E. Hu, R. Hesketh, and K. M. Brindle, "Single shot threedimensional pulse sequence for hyperpolarized 13 C MRI.,” Magn. Reson. Med., vol. 77, no. 2, pp. 740-752, Feb. 2017.

[212] B. J. Geraghty, J. Y. C. Lau, A. P. Chen, and C. H. Cunningham, “Accelerated 3D echo-planar imaging with compressed sensing for time-resolved hyperpolarized 13C studies,” Magn. Reson. Med., vol. 77, no. 2, pp. 538-546, Jan. 2017.

[213] J. J. Miller, A. Z. Lau, I. Teh, J. E. Schneider, P. Kinchecsh, S. Smart, V. Ball, N. Sibson, and D. J. Tyler, "Robust, high resolution three-dimensional hyperpolarised metabolic imaging of the healthy rat heart at 7 T," Magn. Reson. Med., vol. 0, no. 0, p. 0, 2015.

[214] J. M. Wild, N. Woodhouse, M. N. J. Paley, S. Fichele, Z. Said, L. Kasuboski, and E. J. R. van Beek, "Comparison between 2D and 3D gradient-echo sequences for MRI of human lung ventilation with hyperpolarized 3He.,” Magn. Reson. Med., vol. 52, no. 3, pp. 673-8, Sep. 2004.

[215] J. J. Miller, A. Z. Lau, I. Teh, J. E. J. E. Schneider, P. Kinchesh, S. SmarT, V. Ball, N. R. Sibson, and D. J. Tyler, "Robust and high resolution hyperpolarized metabolic imaging of the rat heart at 7T with 3D spectral-spatial EPI,” Magn. Reson. Med., vol. 75, no. 4, pp. 15151524, May 2015.

[216] C. von Morze, P. E. Z. Z. Larson, S. Hu, H. A. I. I. Yoshihara, R. A. Bok, A. Goga, J. H. Ardenkjaer-Larsen, and D. B. Vigneron, "Investigating tumor perfusion and metabolism using multiple hyperpolarized 13C compounds: HP001, pyruvate and urea,” Magn. Reson. Imaging, vol. 30, no. 3, pp. 305-311, Apr. 2012.

[217] P. E. Z. Z. Larson, A. B. Kerr, A. P. Chen, M. S. Lustig, M. L. Zierhut, S. Hu, C. H. Cunningham, J. M. Pauly, J. Kurhanewicz, and D. B. Vigneron, "Multiband excitation pulses for hyperpolarized 13C dynamic chemical-shift imaging,” J. Magn. Reson., vol. 194, no. 1, pp. 121-127, Sep. 2008.

[218] A. Z. Lau, J. Y. C. Lau, A. P. Chen, and C. H. Cunningham, "Simultaneous multislice acquisition without trajectory modification for hyperpolarized13C experiments," Magnetic Resonance in Medicine, 2018. 
[219] J.-N. Dumez and L. Frydman, "Multidimensional excitation pulses based on spatiotemporal encoding concepts,” J. Magn. Reson., vol. 226, pp. 22-34, 2013.

[220] R. Schmidt, C. Laustsen, J.-N. N. Dumez, M. I. Kettunen, E. M. Serrao, I. Marco-Rius, K. M. Brindle, J. H. Ardenkjaer-Larsen, and L. Frydman, "In vivo single-shot 13C spectroscopic imaging of hyperpolarized metabolites by spatiotemporal encoding,” J. Magn. Reson., vol. 240, pp. 8-15, 2014.

[221] P. E. Z. Larson, R. Bok, A. B. Kerr, M. Lustig, S. Hu, A. P. Chen, S. J. Nelson, J. M. Pauly, J. Kurhanewicz, and D. B. Vigneron, "Investigation of tumor hyperpolarized [1-13C]-pyruvate dynamics using time-resolved multiband RF excitation echo-planar MRSI.,” Magn. Reson. Med., vol. 63, no. 3, pp. 582-91, Mar. 2010.

[222] A. Z. Lau, J. J. Miller, and D. J. Tyler, "Mapping of intracellular pH in the in vivo rodent heart using hyperpolarized [1-13C]pyruvate,” Magn. Reson. Med., vol. 77, no. 5, pp. 1810-1817, May 2017.

[223] E. Johansson, L. E. Olsson, S. Månsson, J. S. Petersson, K. Golman, F. Ståhlberg, and R. Wirestam, "Perfusion assessment with bolus differentiation: A technique applicable to hyperpolarized tracers,” Magn. Reson. Med., vol. 52, no. 5, pp. 1043-51, Nov. 2004.

[224] A. Z. Lau, J. J. Miller, M. D. Robson, and D. J. Tyler, “Cardiac perfusion imaging using hyperpolarized 13C urea using flow sensitizing gradients,” Magn. Reson. Med., vol. 75, no. 4, pp. 1474-1483, May 2016.

[225] C. von Morze, S. Sukumar, G. D. Reed, P. E. Z. Larson, R. A. Bok, J. Kurhanewicz, and D. B. Vigneron, "Frequency-specific SSFP for hyperpolarized 13C metabolic imaging at $14.1 \mathrm{~T}$," Magn. Reson. Imaging, vol. 31, no. 2, 2013.

[226] J. Svensson, S. Månsson, E. Johansson, J. S. Petersson, and L. E. Olsson, "Hyperpolarized 13C MR angiography using trueFISP,” Magn. Reson. Med., vol. 50, no. 2, pp. 256-262, Aug. 2003.

[227] L. E. Olsson, C. M. Chai, O. Axelsson, M. Karlsson, K. Golman, and J. S. Petersson, "MR coronary angiography in pigs with intraarterial injections of a hyperpolarized 13C substance," Magn. Reson. Med., vol. 55, no. 4, pp. 731-737, Apr. 2006.

[228] G. D. Reed, C. von Morze, R. Bok, B. L. Koelsch, M. Van Criekinge, K. J. Smith, Hong Shang, P. E. Z. Larson, J. Kurhanewicz, and D. B. Vigneron, "High resolution (13)C MRI with hyperpolarized urea: in vivo T(2) mapping and (15)N labeling effects.," IEEE Trans. Med. Imaging, vol. 33, no. 2, pp. 362-371, Feb. 2014.

[229] K. R. Keshari and D. M. Wilson, "Chemistry and biochemistry of 13C hyperpolarized magnetic resonance using dynamic nuclear polarization.," Chem. Soc. Rev., vol. 43, no. 5, pp. 1627-59, 2014.

[230] T. B. Rodrigues, E. M. Serrao, B. W. C. Kennedy, D.-E. Hu, M. I. Kettunen, and K. M. Brindle, "Magnetic resonance imaging of tumor glycolysis using hyperpolarized 13C-labeled glucose," Nat. Med., vol. 20, no. 1, pp. 93-97, 2013.

[231] K. N. Timm, J. Hartl, M. A. Keller, D. E. Hu, M. I. Kettunen, T. B. Rodrigues, M. Ralser, and K. M. Brindle, "Hyperpolarized [U-2H, U-13C]Glucose reports on glycolytic and pentose phosphate pathway activity in EL4 tumors and glycolytic activity in yeast cells," Magn. Reson. Med., vol. 74, no. 6, pp. 1543-1547, 2015.

[232] M. Mishkovsky, B. Anderson, M. Karlsson, M. H. Lerche, A. D. Sherry, R. Gruetter, Z. Kovacs, and A. Comment, "Measuring glucose cerebral metabolism in the healthy mouse using hyperpolarized ${ }^{13} \mathrm{C}$ magnetic resonance," Sci. Rep., vol. 7, no. 1, 2017.

[233] J. M. Park, M. Wu, K. Datta, S. C. Liu, A. Castillo, H. Lough, D. M. Spielman, and K. L. Billingsley, "Hyperpolarized Sodium [1-13C]-Glycerate as a Probe for Assessing Glycolysis 
in Vivo,” J. Am. Chem. Soc., vol. 139, no. 19, pp. 6629-6634, 2017.

[234] K. X. Moreno, C. E. Harrison, M. E. Merritt, Z. Kovacs, C. R. Malloy, and A. Dean Sherry, "Hyperpolarized $\delta$-[1-13C]gluconolactone as a probe of the pentose phosphate pathway," NMR Biomed., vol. 30, no. 6, 2017.

[235] I. Marco-Rius, C. von Morze, R. Sriram, P. Cao, G. Chang, E. Milshteyn, R. A. Bok, M. A. Ohliger, D. Pearce, J. Kurhanewicz, P. E. Z. Larson, D. B. Vigneron, and M. Merritt, "Monitoring acute metabolic changes in the liver and kidneys induced by fructose and glucose using hyperpolarized [2-(13) C]dihydroxyacetone.,” Magn. Reson. Med., vol. 77, no. 1, pp. 65-73, 2017.

[236] K. X. Moreno, S. Satapati, R. J. DeBerardinis, S. C. Burgess, C. R. Malloy, and M. E. Merritt, "Real-time detection of hepatic gluconeogenic and glycogenolytic states using hyperpolarized [2-13c]dihydroxyacetone,” J. Biol. Chem., vol. 289, no. 52, pp. 35859-35867, 2014.

[237] N. Couto, J. Wood, and J. Barber, "The role of glutathione reductase and related enzymes on cellular redox homoeostasis network,” Free Radical Biology and Medicine, vol. 95. pp. 27-42, 2016.

[238] T. Šimůnek, M. Štěrba, O. Popelová, M. Adamcová, R. Hrdina, and V. Gerši, “Anthracyclineinduced cardiotoxicity: Overview of studies examining the roles of oxidative stress and free cellular iron,” in Pharmacological Reports, 2009, vol. 61, no. 1, pp. 154-171.

[239] R. Ferrari, L. Agnoletti, L. Comini, G. Gaia, T. Bachetti, A. Cargnoni, C. Ceconi, S. Curello, and O. Visioli, “Oxidative stress during myocardial ischaemia and heart failure," Eur.Heart J., vol. 19 Suppl B, no. 0195-668X, pp. B2-11, 1998.

[240] S. E. Bohndiek, M. I. Kettunen, D. Hu, B. W. C. Kennedy, J. Boren, F. A. Gallagher, and K. M. Brindle, "Hyperpolarized [1- $\left.{ }^{13} \mathrm{C}\right]$-Ascorbic and Dehydroascorbic Acid: Vitamin C as a Probe for Imaging Redox Status in Vivo,” J. Am. Chem. Soc., vol. 133, no. 30, pp. 1179511801, 2011.

[241] K. N. Timm, D.-E. Hu, M. Williams, A. J. Wright, M. I. Kettunen, B. W. C. Kennedy, T. J. Larkin, P. Dzien, I. Marco-Rius, S. E. Bohndiek, and K. M. Brindle, “Assessing Oxidative Stress in Tumors by Measuring the Rate of Hyperpolarized $\left[1{ }^{13}\right.$ C]Dehydroascorbic Acid Reduction Using ${ }^{13}$ C Magnetic Resonance Spectroscopy,” J. Biol. Chem., vol. 292, no. 5, pp. 1737-1748, 2017.

[242] K. R. Keshari, J. Kurhanewicz, R. Bok, P. E. Z. Larson, D. B. Vigneron, and D. M. Wilson, "Hyperpolarized 13C dehydroascorbate as an endogenous redox sensor for in vivo metabolic imaging.,” Proc. Natl. Acad. Sci., vol. 108, no. 46, pp. 18606-18611, 2011.

[243] F. a Gallagher, M. I. Kettunen, S. E. Day, D. Hu, M. Karlsson, A. Gisselsson, M. H. Lerche, and K. M. Brindle, "Detection of tumor glutamate metabolism in vivo using (13)C magnetic resonance spectroscopy and hyperpolarized [1-(13)C]glutamate.,” Magn. Reson. Med., vol. 66, no. 1, pp. 18-23, 2011.

[244] M. Karlsson, P. R. Jensen, R. in ’t Zandt, A. Gisselsson, G. Hansson, J. O. Ø. Duus, S. Meier, and M. H. Lerche, "Imaging of branched chain amino acid metabolism in tumors with hyperpolarized 13C ketoisocaproate.,” Int. J. Cancer, vol. 127, no. 3, pp. 729-36, 2010.

[245] H. A. I. Yoshihara, J. A. M. Bastiaansen, M. Karlsson, M. Lerche, A. Comment, and J. Schwitter, "Hyperpolarized [1-13C]octanoate: a probe of myocardial $\beta$-oxidation," in International Society for Magnetic Resonance in Medicine, 2015, p. 0091.

[246] F. A. Gallagher, M. I. Kettunen, S. E. Day, D. E. Hu, J. H. rdenkjaer-Larsen, R. in’t Zandt, P. R. Jensen, M. Karlsson, K. Golman, M. H. Lerche, and K. M. Brindle, "Magnetic resonance imaging of pH in vivo using hyperpolarized C-13-labelled bicarbonate,” Nature, vol. 453, no. 
7197, pp. 940-U73, 2008.

[247] S. Düwel, C. Hundshammer, M. Gersch, B. Feuerecker, K. Steiger, A. Buck, A. Walch, A. Haase, S. J. Glaser, M. Schwaiger, and F. Schilling, "Imaging of $\mathrm{pH}$ in vivo using hyperpolarized 13C-labelled zymonic acid,” Nat. Commun., vol. 8, p. 15126, 2017.

[248] D. E. Korenchan, C. Taglang, C. von Morze, J. E. Blecha, J. W. Gordon, R. Sriram, P. E. Z. Larson, D. B. Vigneron, H. F. VanBrocklin, J. Kurhanewicz, D. M. Wilson, and R. R. Flavell, "Dicarboxylic acids as $\mathrm{pH}$ sensors for hyperpolarized ${ }^{13} \mathrm{C}$ magnetic resonance spectroscopic imaging,” Analyst, vol. 142, no. 9, pp. 1429-1433, 2017.

[249] B. Feuerecker, M. Durst, M. Michalik, G. Schneider, D. Saur, M. Menzel, M. Schwaiger, and F. Schilling, "Hyperpolarized 13C Diffusion MRS of Co-Polarized Pyruvate and Fumarate to Measure Lactate Export and Necrosis.,” J. Cancer, vol. 8, no. 15, pp. 3078-3085, 2017.

[250] M. C. Cassidy, H. R. Chan, B. D. Ross, P. K. Bhattacharya, and C. M. Marcus, "In vivo magnetic resonance imaging of hyperpolarized silicon particles,” Nat. Nanotechnol., vol. 8, no. 5, pp. 363-368, 2013.

[251] A. B. Barnes, E. Markhasin, E. Daviso, V. K. Michaelis, E. A. Nanni, S. K. Jawla, E. L. Mena, R. Derocher, A. Thakkar, P. P. Woskov, J. Herzfeld, R. J. Temkin, and R. G. Griffin, "Dynamic nuclear polarization at $700 \mathrm{MHz} / 460 \mathrm{GHz}$," J. Magn. Reson., vol. 224, pp. 1-7, 2012.

[252] S. Reynolds, S. Metcalf, E. J. Cochrane, R. C. Collins, S. Jones, M. N. J. Paley, and G. M. Tozer, "Direct arterial injection of hyperpolarized13C-labeled substrates into rat tumors for rapid MR detection of metabolism with minimal substrate dilution,” Magn. Reson. Med., vol. 78, no. 6, pp. 2116-2126, 2017.

[253] R. E. Hurd, Y.-F. Yen, D. Mayer, A. Chen, D. Wilson, S. Kohler, R. Bok, D. Vigneron, J. Kurhanewicz, J. Tropp, D. Spielman, and A. Pfefferbaum, "Metabolic imaging in the anesthetized rat brain using hyperpolarized [1-13C] pyruvate and [1-13C] ethyl pyruvate," Magn. Reson. Med., vol. 63, no. 5, pp. 1137-1143, 2010.

[254] H. Allouche-Arnon, A. Gamliel, R. Nalbandian, M. Mishkovsky, L. Frydman, J. Gomori, R. Lenkinski, C. Barzilay, and R. Katz-Brull, "Effects of deuteration on 13C relaxation times in neuro-metabolic compounds: Implications for hyperpolarized spectroscopic imaging," in Proceedings 17th Scientific Meeting, International Society for Magnetic Resonance in Medicine, 2009, vol. Honolulu, p. 2442.

[255] I. Marco-Rius, M. C. D. Tayler, M. I. Kettunen, T. J. Larkin, K. N. Timm, E. M. Serrao, T. B. Rodrigues, G. Pileio, J. H. Ardenkjaer-Larsen, M. H. Levitt, and K. M. Brindle, "Hyperpolarized singlet lifetimes of pyruvate in human blood and in the mouse," NMR Biomed., vol. 26, no. 12, pp. 1696-1704, 2013.

[256] G. Pileio, M. Carravetta, and M. H. Levitt, "Storage of nuclear magnetization as long-lived singlet order in low magnetic field,” Proc. Natl. Acad. Sci., vol. 107, no. 40, pp. 17135-17139, 2010.

[257] T. R. Eichhorn, Y. Takado, N. Salameh, A. Capozzi, T. Cheng, J.-N. Hyacinthe, M. Mishkovsky, C. Roussel, and A. Comment, "Hyperpolarization without persistent radicals for in vivo real-time metabolic imaging," Proc. Natl. Acad. Sci., vol. 110, no. 45, pp. 1806418069, 2013.

[258] J. A. M. Bastiaansen, H. A. I. Yoshihara, A. Capozzi, J. Schwitter, R. Gruetter, M. E. Merritt, and A. Comment, "Probing cardiac metabolism by hyperpolarized 13C MR using an exclusively endogenous substrate mixture and photo-induced nonpersistent radicals," Magnetic Resonance in Medicine, 2018. 
[259] A. Capozzi, T. Cheng, G. Boero, C. Roussel, and A. Comment, "Thermal annihilation of photo-induced radicals following dynamic nuclear polarization to produce transportable frozen hyperpolarized 13 C-substrates,” Nat. Commun., vol. 8, 2017.

[260] M. Batel, M. Krajewski, K. Weiss, O. With, A. D??pp, A. Hunkeler, M. Gimersky, K. P. Pruessmann, P. Boesiger, B. H. Meier, S. Kozerke, and M. Ernst, "A multi-sample $94 \mathrm{GHz}$ dissolution dynamic-nuclear-polarization system,” J. Magn. Reson., vol. 214, pp. 166-174, 2012.

[261] M. Krajewski, P. Wespi, J. Busch, L. Wissmann, G. Kwiatkowski, J. Steinhauser, M. Batel, M. Ernst, and S. Kozerke, "A multisample dissolution dynamic nuclear polarization system for serial injections in small animals,” Magn. Reson. Med., vol. 77, no. 2, pp. 904-910, 2017.

[262] J. Steinhauser, P. Wespi, G. Kwiatkowski, and S. Kozerke, "Assessing the influence of isoflurane anesthesia on cardiacmetabolism using hyper polarized [1-13C]pyruvate," NMR Biomed., 2017.

[263] H. Gutte, A. E. Hansen, M. M. E. Larsen, S. Rahbek, S. T. Henriksen, H. H. Johannesen, J. Ardenkjaer-Larsen, A. T. Kristensen, L. Hojgaard, and A. Kjaer, "Simultaneous Hyperpolarized 13C-Pyruvate MRI and 18F-FDG PET (HyperPET) in 10 Dogs with Cancer,” J. Nucl. Med., vol. 56, no. 11, pp. 1786-1792, 2015.

[264] A. Eldirdiri, A. Clemmensen, S. Bowen, A. Kjær, and J. H. Ardenkjær-Larsen, "Simultaneous imaging of hyperpolarized $\left[1,4-{ }^{13} \mathrm{C}<\mathrm{inf}>2<\right.$ inf $>$ fumarate, $\left[1-{ }^{13} \mathrm{C}\right]$ pyruvate and ${ }^{18} \mathrm{~F}$-FDG in a rat model of necrosis in a clinical PET/MR scanner,” NMR Biomed., 2017.

[265] H. Gutte, A. E. Hansen, S. T. Henriksen, H. H. Johannesen, J. Ardenkjaer-Larsen, A. Vignaud, A. E. Hansen, B. Børresen, T. L. Klausen, A.-M. N. Wittekind, N. Gillings, A. T. Kristensen, A. Clemmensen, L. Højgaard, and A. Kjær, "Simultaneous hyperpolarized (13)C-pyruvate MRI and (18)F-FDG-PET in cancer (hyperPET): feasibility of a new imaging concept using a clinical PET/MRI scanner.,” Am. J. Nucl. Med. Mol. Imaging, vol. 5, no. 1, pp. 38-45, 2015.

[266] L. M. Le Page, D. R. Ball, V. Ball, M. S. Dodd, J. J. Miller, L. C. Heather, and D. J. Tyler, "Simultaneous in vivo assessment of cardiac and hepatic metabolism in the diabetic rat using hyperpolarized MRS,” NMR Biomed., vol. 29, no. 12, pp. 1759-1767, 2016.

[267] D. J. Tyler, M. A. Schroeder, L. E. Cochlin, K. Clarke, and G. K. Radda, “Application of Hyperpolarized Magnetic Resonance in the Study of Cardiac Metabolism,” Appl. Magn. Reson., vol. 34, no. 3-4, pp. 523-531, Aug. 2008.

[268] M. A. Schroeder, L. E. Cochlin, L. C. Heather, K. Clarke, G. K. Radda, and D. J. Tyler, “In vivo assessment of pyruvate dehydrogenase flux in the heart using hyperpolarized carbon-13 magnetic resonance.,” Proc. Natl. Acad. Sci. U. S. A., vol. 105, no. 33, pp. 12051-6, Aug. 2008.

[269] M. A. Schroeder, A. Z. Lau, A. P. Chen, Y. Gu, J. Nagendran, J. Barry, X. Hu, J. R. B. Dyck, D. J. Tyler, K. Clarke, K. A. Connelly, G. A. Wright, and C. H. Cunningham, "Hyperpolarized (13)C magnetic resonance reveals early- and late-onset changes to in vivo pyruvate metabolism in the failing heart.,” Eur. J. Hear. Fail., vol. 15, no. 2, pp. 130-140, Feb. 2013.

[270] J. A. M. M. Bastiaansen, T. Cheng, M. Mishkovsky, J. M. N. J. M. N. Duarte, A. Comment, and R. Gruetter, "In vivo enzymatic activity of acetylCoA synthetase in skeletal muscle revealed by 13C turnover from hyperpolarized [1-13C] acetate to [1-13C]acetylcarnitine,” Biochim. Biophys. Acta - Gen. Subj., vol. 1830, no. 8, pp. 4171-4178, Aug. 2013.

[271] D. R. Ball, B. Rowlands, M. S. Dodd, L. Le Page, V. Ball, C. A. Carr, K. Clarke, and D. J. Tyler, "Hyperpolarized butyrate: A metabolic probe of short chain fatty acid metabolism in the heart,” Magn. Reson. Med., vol. 71, no. 5, pp. 1663-1669, May 2014. 
[272] M. E. Merritt, C. Harrison, C. Storey, F. M. Jeffrey, A. D. Sherry, and C. R. Malloy, "Hyperpolarized 13C allows a direct measure of flux through a single enzyme-catalyzed step by NMR.,” Proc. Natl. Acad. Sci. U. S. A., vol. 104, no. 50, pp. 19773-19777, Dec. 2007.

[273] F. A. Gallagher, M. I. Kettunen, S. E. Day, D.-E. Hu, J. H. Ardenkjaer-Larsen, R. in 't Zandt, P. R. Jensen, M. Karlsson, K. Golman, M. H. Lerche, and K. M. Brindle, "Magnetic resonance imaging of $\mathrm{pH}$ in vivo using hyperpolarized 13C-labelled bicarbonate.," Nature, vol. 453, no. 7197, pp. 940-3, Jun. 2008.

[274] K. Golman, J. S. Petersson, P. Magnusson, E. Johansson, P. Akeson, C.-M. M. Chai, G. Hansson, S. Månsson, P. Åkeson, C.-M. M. Chai, G. Hansson, S. Månsson, P. Akeson, C.-M. M. Chai, G. Hansson, and S. Månsson, "Cardiac metabolism measured noninvasively by hyperpolarized 13C MRI,” Magn. Reson. Med., vol. 59, no. 5, pp. 1005-1013, May 2008.

[275] M. Marjańska, I. Iltis, A. A. Shestov, D. K. Deelchand, C. Nelson, K. Uurbil, P.-G. G. Henry, K. Uğurbil, and P.-G. G. Henry, "In vivo 13C spectroscopy in the rat brain using hyperpolarized [1-13C]pyruvate and [2-13C]pyruvate,” J. Magn. Reson., vol. 206, no. 2, pp. 210-218, 2010.

[276] S. Yang, J. Lee, E. Joe, H. Lee, Y. S. Choi, J. M. Park, D. Spielman, H. T. Song, and D. H. Kim, "Metabolite-selective hyperpolarized 13C imaging using extended chemical shift displacement at 9.4 T,” Magn. Reson. Imaging, vol. 34, no. 4, pp. 535-540, May 2016.

[277] C. H. Cunningham, A. P. Chen, M. J. Albers, J. Kurhanewicz, R. E. Hurd, Y. F. Yen, J. M. Pauly, S. J. Nelson, and D. B. Vigneron, "Double spin-echo sequence for rapid spectroscopic imaging of hyperpolarized 13C,” J. Magn. Reson., vol. 187, no. 2, pp. 357-362, Aug. 2007.

[278] M. R. Clatworthy, M. I. Kettunen, D.-E. Hu, R. J. Mathews, T. H. Witney, B. W. C. Kennedy, S. E. Bohndiek, F. A. Gallagher, L. B. Jarvis, K. G. C. Smith, and K. M. Brindle, "Magnetic resonance imaging with hyperpolarized [1,4-(13)C2]fumarate allows detection of early renal acute tubular necrosis.," Proc. Natl. Acad. Sci. U. S. A., vol. 109, no. 33, pp. 13374-13379, Aug. 2012.

[279] K. Golman, R. in 't Zandt, and M. Thaning, "Real-time metabolic imaging.," Proc. Natl. Acad. Sci. U. S. A., vol. 103, no. 30, pp. 11270-5, Jul. 2006.

[280] K. Golman and J. S. Petersson, "Metabolic imaging and other applications of hyperpolarized 13C1.,” Acad. Radiol., vol. 13, no. 8, pp. 932-942, Aug. 2006.

[281] M. Durst, U. Koellisch, A. Frank, G. Rancan, C. V. Gringeri, V. Karas, F. Wiesinger, M. I. Menzel, M. Schwaiger, A. Haase, and R. F. Schulte, "Comparison of acquisition schemes for hyperpolarised 13C imaging,” NMR Biomed., vol. 28, no. 6, pp. 715-725, Jun. 2015.

[282] F. Wiesinger, E. Weidl, M. I. Menzel, M. A. Janich, O. Khegai, S. J. Glaser, A. Haase, M. Schwaiger, and R. F. Schulte, "IDEAL spiral CSI for dynamic metabolic MR imaging of hyperpolarized [1-13C]pyruvate.,” Magn. Reson. Med., vol. 68, no. 1, pp. 8-16, Jul. 2012.

[283] O. Khegai, R. F. Schulte, M. A. Janich, M. I. Menzel, E. Farrell, A. M. Otto, J. H. ArdenkjaerLarsen, S. J. Glaser, A. Haase, M. Schwaiger, and F. Wiesinger, “Apparent rate constant mapping using hyperpolarized [1-(13)C]pyruvate.,” NMR Biomed., vol. 27, no. 10, pp. 125665, Oct. 2014.

[284] C. von Morze, R. A. Bok, G. D. Reed, J. H. Ardenkjaer-Larsen, J. Kurhanewicz, and D. B. Vigneron, "Simultaneous multiagent hyperpolarized (13)C perfusion imaging.," Magn. Reson. Med., vol. 72, no. 6, pp. 1599-1609, Dec. 2014.

[285] P. E. Z. Larson, R. Bok, A. B. Kerr, M. Lustig, S. Hu, A. P. Chen, S. J. Nelson, J. M. Pauly, J. Kurhanewicz, and D. B. Vigneron, "Investigation of tumor hyperpolarized [1-13C]-pyruvate dynamics using time-resolved multiband RF excitation echo-planar MRSI.,” Magn. Reson. 
Med., vol. 63, no. 3, pp. 582-91, Mar. 2010.

[286] Y. Zur, "Design of improved spectral-spatial pulses for routine clinical use.," Magn. Reson. Med., vol. 43, no. 3, pp. 410-20, Mar. 2000.

[287] C.-Y. Yip, D. Yoon, V. Olafsson, S. Lee, W. A. Grissom, J. A. Fessler, and D. C. Noll, "Spectral-spatial pulse design for through-plane phase precompensatory slice selection in T2*weighted functional MRI.,” Magn. Reson. Med., vol. 61, no. 5, pp. 1137-1147, May 2009.

[288] S. Hu, M. Lustig, A. P. Chen, J. Crane, A. Kerr, D. A. C. C. C. Kelley, R. Hurd, J. Kurhanewicz, S. J. Nelson, J. M. Pauly, and D. B. Vigneron, "Compressed sensing for resolution enhancement of hyperpolarized 13C flyback 3D-MRSI.,” J. Magn. Reson., vol. 192, no. 2, pp. 258-264, Jun. 2008.

[289] A. Z. Lau, A. P. Chen, R. E. Hurd, and C. H. Cunningham, "Spectral-spatial excitation for rapid imaging of DNP compounds.,” NMR Biomed., vol. 24, no. 8, pp. 988-96, Oct. 2011.

[290] R. F. Schulte, J. I. Sperl, E. Weidl, M. I. Menzel, M. A. Janich, O. Khegai, M. Durst, J. H. Ardenkjaer-Larsen, S. J. Glaser, A. Haase, M. Schwaiger, and F. Wiesinger, "Saturationrecovery metabolic-exchange rate imaging with hyperpolarized [1-(13) C] pyruvate using spectral-spatial excitation.,” Magn. Reson. Med., vol. 69, no. 5, pp. 1209-16, May 2012.

[291] J. Wang, A. J. Wright, D.-E. Hu, R. Hesketh, and K. M. Brindle, "Single shot threedimensional pulse sequence for hyperpolarized 13 C MRI.,” Magn. Reson. Med., vol. 77, no. 2, pp. 740-752, Feb. 2017.

[292] M. A. Schroeder, L. E. Cochlin, L. C. Heather, K. Clarke, G. K. Radda, and D. J. Tyler, "In vivo assessment of pyruvate dehydrogenase flux in the heart using hyperpolarized carbon-13 magnetic resonance.," Proc. Natl. Acad. Sci. U. S. A., vol. 105, no. 33, pp. 12051-6, Aug. 2008.

[293] M. A. Schroeder, A. Z. Lau, A. P. Chen, Y. Gu, J. Nagendran, J. Barry, X. Hu, J. R. B. Dyck, D. J. Tyler, K. Clarke, K. A. Connelly, G. A. Wright, and C. H. Cunningham, "Hyperpolarized (13)C magnetic resonance reveals early- and late-onset changes to in vivo pyruvate metabolism in the failing heart.,” Eur. J. Hear. Fail., vol. 15, no. 2, pp. 130-140, Feb. 2013. 


\section{Glossary}

${ }^{13} \mathrm{C}$ : carbon-13

${ }^{1} \mathrm{H}$ : proton, hydrogen-1

${ }^{31} \mathrm{P}$ : phosphorus-31

${ }^{29}$ Si: silicon-29

AAB: abdominal aortic banding

ALT: alanine aminotransferase

ATP: adenosine triphosphate

CMR: cardiac magnetic resonance

CPT-1: carnitine-palmitoyltransferase 1

CSI: chemical shift imaging

CT: computed tomography

DCA: dichloroacetate

DCM: dilated cardiomyopathy

DE: differential equation

DHA: dehydroascorbic acid

DNP: dynamic nuclear polarisation

EPSI: echo planar spectroscopic imaging

FAT: fatty acid transporter

FFA: free fatty acid

GLUT: glucose transporter

ISIS: image selected in vivo spectroscopy

LASER: localisation via adiabatic spin echo refocussing

LDH: lactate dehydrogenase

LGE: late gadolinium enhancement

MI: myocardial infarction

MR: magnetic resonance

MRI: magnetic resonance imaging

MRS: magnetic resonance spectroscopy

NMR: nuclear magnetic resonance

PCr: phosphocreatine

PDH: pyruvate dehydrogenase 
PET: positron emission tomography

$\mathrm{pH}_{\mathrm{i}}$ : intracellular $\mathrm{pH}$

PPP: pentose phosphate pathway

PRESS: point resolved spectroscopy

SHR: spontaneously hypertensive rats

SNR: signal-to-noise ratio

SPECT: single photon emission computed tomography

STEAM: stimulated echo acquisition mode

TCA: tricarboxylic acid 


\begin{tabular}{|c|c|c|c|c|}
\hline PATHOLOGY & Setting & Model & Substrate & Reference \\
\hline \multirow{8}{*}{$\begin{array}{c}\text { ISCHAEMIC } \\
\text { HEART } \\
\text { DISEASE }\end{array}$} & Global Ischaemia & $\begin{array}{c}\text { Isolated, perfused rat } \\
\text { heart }\end{array}$ & {$\left[2-{ }^{13} \mathrm{C}\right]$ Pyruvate } & {$[100]$} \\
\hline & Infarction & $\begin{array}{c}\text { Isolated, perfused rat } \\
\text { heart }\end{array}$ & $\begin{array}{l}{\left[1-{ }^{13} \mathrm{C}\right] \text { Pyruvate }} \\
{\left[2-{ }^{13} \mathrm{C}\right] \text { Pyruvate }}\end{array}$ & $\begin{array}{l}{[130]} \\
{[112]}\end{array}$ \\
\hline & Infarction & In vivo rat & $\begin{array}{c}{\left[1-{ }^{13} \mathrm{C}\right] \text { Pyruvate } \&} \\
{\left[2-{ }^{13} \mathrm{C}\right] \text { Pyruvate }}\end{array}$ & [133] \\
\hline & $\begin{array}{c}\text { Infarction - role of } \\
\text { macrophages }\end{array}$ & In vivo rat and pig & {$\left[1-{ }^{13} \mathrm{C}\right]$ Pyruvate } & [159] \\
\hline & $\begin{array}{c}\text { Infarction - } \\
\text { necrosis }\end{array}$ & In vivo rat & {$\left[1,4-{ }^{13} \mathrm{C}_{2}\right]$ Fumarate } & [142] \\
\hline & Reperfusion & $\begin{array}{c}\text { Isolated, perfused rat } \\
\text { heart }\end{array}$ & {$\left[1-{ }^{13} \mathrm{C}\right]$ Pyruvate } & [129] \\
\hline & Reperfusion & In vivo rat & {$\left[1-{ }^{13} \mathrm{C}\right]$ Pyruvate } & $\begin{array}{l}{[131],} \\
{[132]}\end{array}$ \\
\hline & $\begin{array}{l}\text { Myocardial } \\
\text { viability }\end{array}$ & In vivo pig & {$\left[1-^{-13} \mathrm{C}\right]$ Pyruvate } & [140] \\
\hline \multirow{6}{*}{$\begin{array}{c}\text { NON- } \\
\text { ISCHAEMIC } \\
\text { HEART } \\
\text { DISEASE }\end{array}$} & Hypertrophy & $\begin{array}{c}\text { In vivo rat, } \\
\text { Hyperthyroidism }\end{array}$ & $\begin{array}{c}{\left[1-{ }^{13} \mathrm{C}\right] \text { Pyruvate }} \\
\&\left[2-{ }^{13} \mathrm{C}\right] \text { Pyruvate }\end{array}$ & [114] \\
\hline & Hypertrophy & $\begin{array}{c}\text { Spontaneously } \\
\text { Hypertensive Rat, in } \\
\text { vivo }\end{array}$ & $\begin{array}{c}{\left[1{ }^{-13} \mathrm{C}\right] \text { Pyruvate }} \\
\&\left[2-{ }^{13} \mathrm{C}\right] \text { Pyruvate }\end{array}$ & {$[144]$} \\
\hline & Hypertrophy & $\begin{array}{c}\text { In vivo rat, } \\
\text { Abdominal Aortic } \\
\text { Banding }\end{array}$ & $\begin{array}{c}{\left[1-{ }^{13} \mathrm{C}\right] \text { Pyruvate }} \\
\&\left[2-{ }^{13} \mathrm{C}\right] \text { Pyruvate }\end{array}$ & {$[146]$} \\
\hline & $\begin{array}{c}\text { Dilated } \\
\text { Cardiomyopathy }\end{array}$ & $\begin{array}{l}\text { In vivo pig, pacing- } \\
\text { induced DCM }\end{array}$ & $\begin{array}{c}{\left[1-{ }^{13} \mathrm{C}\right] \text { Pyruvate }} \\
\&\left[2-{ }^{13} \mathrm{C}\right] \text { Pyruvate }\end{array}$ & [147] \\
\hline & Diabetes & $\begin{array}{c}\text { In vivo rat, type } 1 \\
\text { diabetes }\end{array}$ & {$\left[1-{ }^{13} \mathrm{C}\right]$ Pyruvate } & [103] \\
\hline & Diabetes & $\begin{array}{c}\text { In vivo rat, type } 2 \\
\text { diabetes }\end{array}$ & {$\left[1-{ }^{13} \mathrm{C}\right]$ Pyruvate } & $\begin{array}{l}{[150],} \\
{[266]}\end{array}$ \\
\hline
\end{tabular}

Table 1 List of preclinical models of heart disease explored by hyperpolarised magnetic resonance 


\begin{tabular}{|c|c|c|c|c|}
\hline $\begin{array}{c}\text { Sequence } \\
\text { Name }\end{array}$ & Brief Description & Strengths & Limitations & Refs. \\
\hline $\begin{array}{l}\text { Unlocalised } \\
\text { spectroscopy }\end{array}$ & $\begin{array}{l}\text { Hard or adiabatic } \\
\text { pulse/acquire } \\
\text { spectroscopy, } \\
\text { localised only by } \\
\text { coil placement }\end{array}$ & $\begin{array}{c}\text { - } \quad \text { Straightforward and } \\
\text { robust } \\
\text { - } \quad \text { Gold standard” for } \\
\text { spectroscopy } \\
\text { - Spectral } \\
\text { quantification } \\
\text { algorithms are well } \\
\text { established } \\
\text { - } \quad \text { High temporal and } \\
\text { spectral resolution } \\
\text { Wide compound } \\
\text { applicability }\end{array}$ & $\begin{array}{l}\text { - No spatial } \\
\text { information provided } \\
\text { beyond coil } \\
\text { localisation } \\
\text { - If volume coils are } \\
\text { used for reception, } \\
\text { low SNR. If surface } \\
\text { coils are used for } \\
\text { reception, uncertain } \\
\text { spatial localisation. } \\
\text { The necessary use of } \\
\text { low flip angles at } \\
\text { early times may } \\
\text { preclude the detection } \\
\text { of small metabolite } \\
\text { peaks }\end{array}$ & $\begin{array}{c}{[110],} \\
{[112],} \\
{[274],} \\
{[275],} \\
{[156],} \\
{[267]-} \\
{[273]}\end{array}$ \\
\hline $\begin{array}{c}\text { 1D } \\
\text { Spectroscopy }\end{array}$ & $\begin{array}{l}\text { Slice selective } \\
\text { spectroscopy, } \\
\text { additionally } \\
\text { localised by coil } \\
\text { placement }\end{array}$ & $\begin{array}{cc}\text { - } & \text { Straightforward and } \\
\text { robust } \\
\text { - } \quad \text { Provides 1D } \\
\text { localisation } \\
\text { - } \quad \text { Enables detection of } \\
\text { spectra from spatially } \\
\text { discontinuous regions } \\
\text { - } \quad \text { Wide compound } \\
\text { applicability } \\
\text { - High temporal and } \\
\text { spectral resolution } \\
\text { (depending on } \\
\text { bandwidth) }\end{array}$ & $\begin{array}{l}\text { - Highly limited spatial } \\
\text { resolution } \\
\text { - } \quad \text { Slice selection may } \\
\text { alter spectral } \\
\text { lineshapes } \\
\text { - Depending on the } \\
\text { exact protocol and } \\
\text { coil used, spatial } \\
\text { aliasing may be } \\
\text { problematic } \\
\text { - Unknown spatial } \\
\text { weighting by coil } \\
\text { sensitivity }\end{array}$ & $\begin{array}{l}{[266],} \\
{[276]}\end{array}$ \\
\hline LASER & $\begin{array}{l}\text { Localisation by } \\
\text { adiabatic spin echo } \\
\text { refocussing }\end{array}$ & 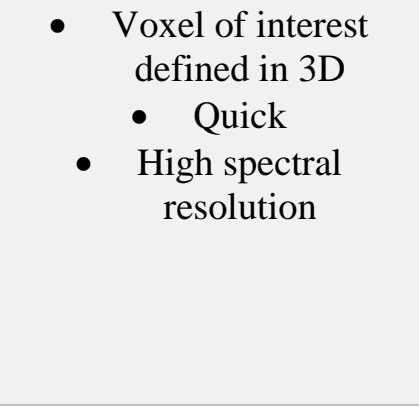 & $\begin{array}{l}\text { Potentially lower } \\
\text { SNR than other } \\
\text { techniques } \\
\text { - Very sensitive to flow } \\
\text { and coil geometry } \\
\text { - Potential for errant } \\
\text { high FA pulses to end } \\
\text { the experiment } \\
\text { prematurely }\end{array}$ & $\begin{array}{l}{[202],} \\
{[275],} \\
{[277]}\end{array}$ \\
\hline FID CSI & $\begin{array}{l}\text { “Conventional” } \\
\text { chemical shift } \\
\text { imaging, with } \\
\text { localisation } \\
\text { obtained through } \\
\text { phase encoding }\end{array}$ & $\begin{array}{c}\text { Better spatial } \\
\text { resolution than } \\
\text { localised } \\
\text { spectroscopic } \\
\text { techniques } \\
\text { - } \quad \text { Potentially higher } \\
\text { SNR than single } \\
\text { voxel spectroscopy } \\
\text { - Fully sampled spectra }\end{array}$ & $\begin{array}{c}\text { - Compromised } \\
\text { spectral, spatial and } \\
\text { temporal resolution } \\
\text { - Slow, usually only } \\
\text { yields a single } \\
\text { timepoint } \\
\text { - } \quad \text { Large number of RF } \\
\text { pulses required } \\
\text { degrades }\end{array}$ & $\begin{array}{c}{[278]-} \\
{[280]}\end{array}$ \\
\hline
\end{tabular}




\begin{tabular}{|c|c|c|c|c|}
\hline & & $\begin{array}{c}\text { are acquired } \\
\text { - Benign and calculable } \\
\text { image-domain point } \\
\text { spread function } \\
\text { - "Gold standard" } \\
\text { method of performing } \\
\text { chemical shift } \\
\text { imaging }\end{array}$ & $\begin{array}{c}\text { magnetisation } \\
\text { - } \quad \text { Large number of RF } \\
\text { pulses degrades slice } \\
\text { profile and applies } \\
\text { filter to k-space data } \\
\text { - } \quad \text { Highly limited matrix } \\
\text { size }\end{array}$ & \\
\hline $\begin{array}{l}\text { Accelerated } \\
\text { forms of CSI }\end{array}$ & $\begin{array}{l}\text { CSI with a non- } \\
\text { Cartesian readout } \\
\text { (e.g. EPSI or spiral } \\
\text { CSI) }\end{array}$ & $\begin{array}{l}\text { - As for CSI, but with a } \\
\text { reduced number of } \\
\text { RF excitations and } \\
\text { increased temporal } \\
\text { (or hence spatial) } \\
\text { resolution }\end{array}$ & $\begin{array}{l}\text { As for CSI, but with } \\
\text { potentially inferior } \\
\text { PSF, improved } \\
\text { temporal resolution, } \\
\text { and rapid imaging } \\
\text { related artefacts. For a } \\
\text { quantitative } \\
\text { comparison with CSI, } \\
\text { see [281]. }\end{array}$ & [277] \\
\hline IDEAL CSI & $\begin{array}{l}\text { Broadband } \\
\text { excitation followed } \\
\text { by a rapid imaging } \\
\text { readout with } \\
\text { iterated echo times } \\
\text { (typically echo } \\
\text { times are acquired } \\
\text { for } n \text { peaks, with an } \\
\text { additional FID } \\
\text { acquisition). }\end{array}$ & 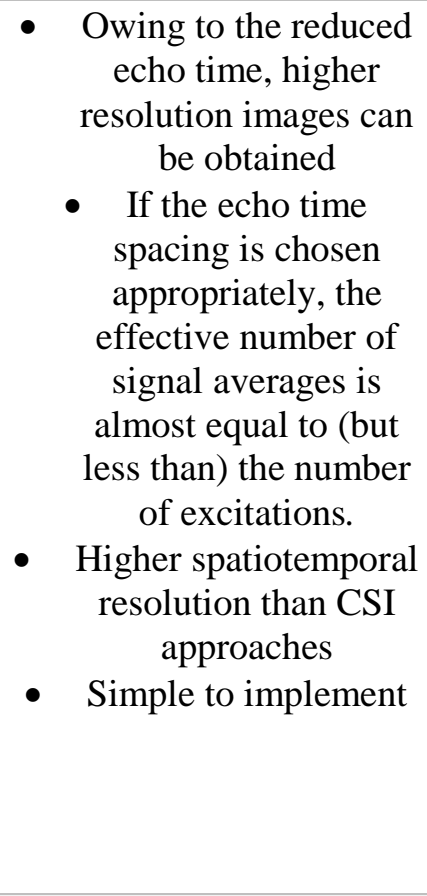 & $\begin{array}{l}\text { - Spectral resolution is } \\
\text { limited and has to be } \\
\text { decided } a \text { priori } \\
\text { - All peaks are excited } \\
\text { with the same flip } \\
\text { angle } \\
\text {-Bleed through } \\
\text { ringing” can occur } \\
\text { when one peak is } \\
\text { much larger than } \\
\text { others } \\
\text { - Rapid imaging } \\
\text { readout can introduce } \\
\text { artefacts which, } \\
\text { owing to the presence } \\
\text { of multiple peaks, can } \\
\text { be difficult to } \\
\text { ameliorate } \\
\text { Complex } \\
\text { reconstruction }\end{array}$ & $\begin{array}{l}{[282],} \\
{[283]}\end{array}$ \\
\hline $\begin{array}{l}\text { Multiband } \\
\text { IDEAL CSI }\end{array}$ & $\begin{array}{c}\text { Multiband } \\
\text { excitation followed } \\
\text { by echo time } \\
\text { shifting rapid } \\
\text { imaging readout }\end{array}$ & $\begin{array}{l}\text { - } \quad \text { As IDEAL CSI, but } \\
\text { with different flip } \\
\text { angle excitation on } \\
\text { different metabolites } \\
\text { - } \quad \text { Potentially more } \\
\text { magnetisation } \\
\text { efficient } \\
\text { - } \quad \text { Potential ability to } \\
\text { acquire equal-SNR } \\
\text { images }\end{array}$ & $\begin{array}{l}\text { - As IDEAL, but more } \\
\text { sensitive to } \Delta \mathrm{B} 0 \text { and } \\
\text { initial transmitter } \\
\text { frequency } \\
\text { - Limited compatibility } \\
\text { with different probes } \\
\text { depending on their } \\
\text { spectrum }\end{array}$ & $\begin{array}{l}{[142],} \\
{[210],} \\
{[217],} \\
{[284],} \\
{[285]}\end{array}$ \\
\hline $\begin{array}{l}\text { Spectral- } \\
\text { Spatial } \\
\text { excitation }\end{array}$ & $\begin{array}{l}\text { Spectrally and } \\
\text { spatially selective } \\
\text { RF excitation } \\
\text { followed by a rapid } \\
\text { imaging readout }\end{array}$ & $\begin{array}{l}\text { - Different compounds } \\
\text { can be excited with } \\
\text { different flip angles } \\
\text { - } \begin{array}{c}\text { Believed to be highly } \\
\text { magnetisation } \\
\text { efficient }\end{array} \\
\text { - } \quad \text { Stock rapid imaging }\end{array}$ & $\begin{array}{l}\text { - } \quad \text { Pulse design is } \\
\text { complex, and specific } \\
\text { for a particular } \\
\text { combination of field } \\
\text { strength, compound, } \\
\text { and available } \mathrm{T}_{2}^{*} \text {. } \\
\text { - } \quad \text { Rapidly oscillating }\end{array}$ & $\begin{array}{l}{[202],} \\
{[210],} \\
{[215],} \\
{[286]-} \\
{[291]}\end{array}$ \\
\hline
\end{tabular}




\begin{tabular}{|c|c|c|c|}
\hline & & $\begin{array}{c}\text { readouts can be used } \\
\text { (EPI, spiral, etc) } \\
\text { - } \quad \text { Reconstruction more } \\
\text { straightforward than } \\
\text { multiecho approaches }\end{array}$ & $\begin{array}{c}\text { slice select gradient } \\
\text { waveform places } \\
\text { limits on minimum } \\
\text { achievable slice } \\
\text { thickness } \\
\text { - Narrow spectral } \\
\text { excitation bandwidth } \\
\text { necessitates } \\
\text { comparatively long } \\
\text { RF }\end{array}$ \\
\hline
\end{tabular}

Table 2: Pulse sequences for hyperpolarised magnetic resonance imaging of metabolism and their properties 


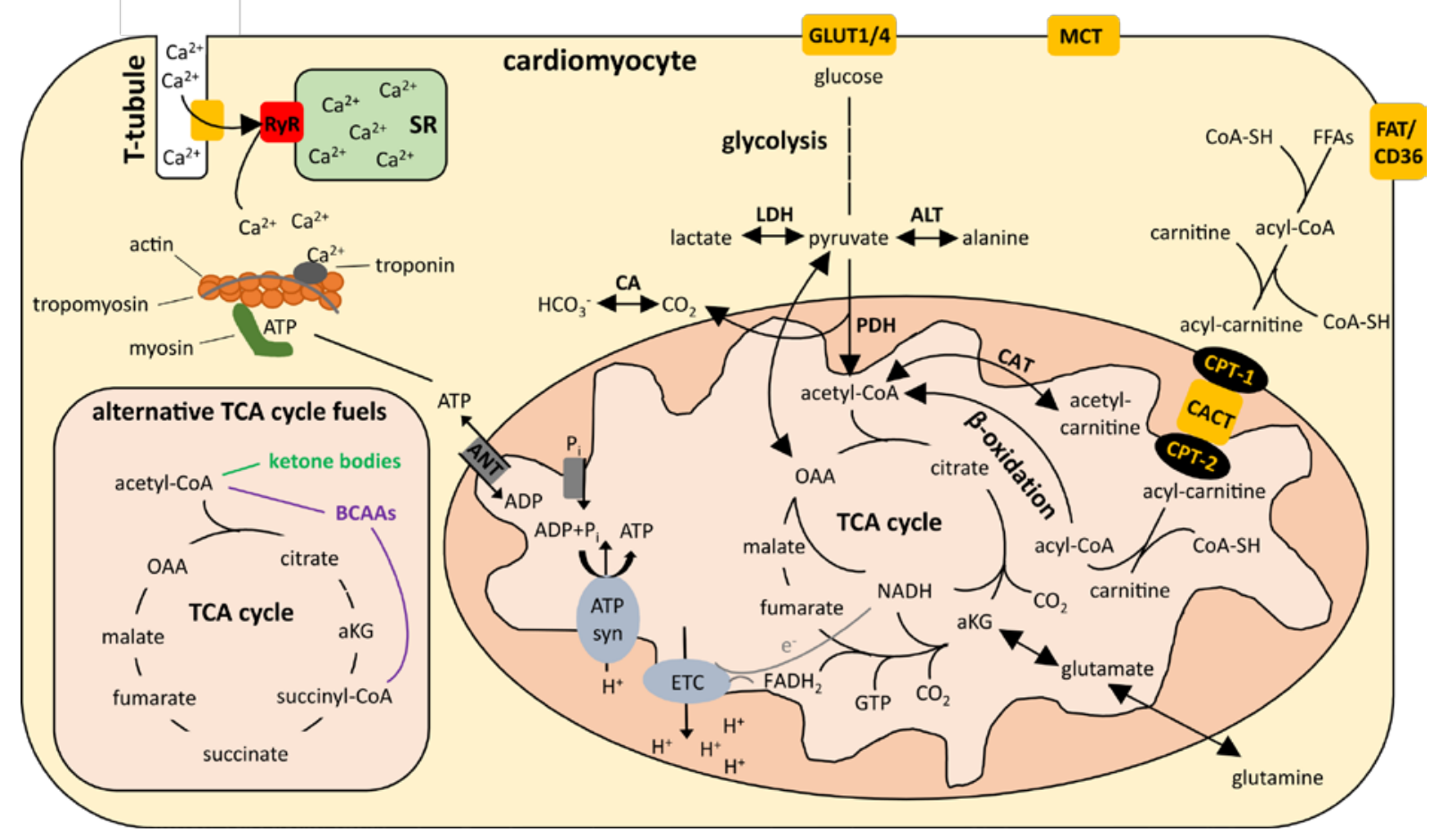

\section{Figure 1 Cardiac metabolism}

The heart uses predominantly free fatty acids (FFAs) and glucose for energy generation. FFAs are transported into the mitochondria via the carnitine shuttle and then oxidised to acetyl-CoA in successive rounds of $\beta$-oxidation. Glucose is oxidised in glycolysis to acetyl-CoA by the gate-keeper enzyme pyruvate dehydrogenase (PDH), yielding carbon dioxide $\left(\mathrm{CO}_{2}\right)$ that is in rapid exchange with bicarbonate $\left(\mathrm{HCO}_{3}^{-}\right)$, or alternatively it is anaerobically broken down to lactate in the cytosol. AcetylCoA is further oxidised in the tricarboxylic acid (TCA) cycle to yield the electron carriers NADH and $\mathrm{FADH}_{2}$. The TCA cycle can also accept alternative substrates such as ketone bodies and branchedchain amino acids (BCAAs) as shown in the inset. The electron carriers then donate electrons to the electron transport chain (ETC) which yields free energy to pump protons into the mitochondrial intermembrane space. The electrochemical gradient so generated leads to the proton motive force, where protons flow back into the mitochondrial matrix through ATP synthase (ATP syn), which generates ATP. The ATP is then transported into the cytosol in exchange for ADP by the adenine nucleotide translocase (ANT). ATP then binds myosin to detach the latter from actin filaments allowing for a new power stroke to be initiated. Action potentials arriving at the cardiomyocytes allow entry of $\mathrm{Ca}^{2+}$ ions into the cells through t-tubular $\mathrm{Ca}^{2+}$ channels. These $\mathrm{Ca}^{2+}$ ions bind onto ryanodine receptors (RyR) on the sarcoplasmic reticulum (SR) mediating $\mathrm{Ca}^{2+}$-induced $\mathrm{Ca}^{2+}$ release. $\mathrm{Ca}^{2+}$ ions then bind troponin, initiating another power stroke at the sarcomere through ATP hydrolysis at the myosin head. Other abbreviations used in this figure: aKG, alpha-ketoglutarate; ALT, alanine aminotransferase; CA, carbonic anhydrase; CAT, carnitine acyltransferase; CACT, carnitineacylcarnitine translocase; CPT, carnitine palmitoyltransferase; FAT, fatty acid transporter; GLUT, glucose transporter; LDH, lactate dehydrogenase; MCT, monocarboxylate transporter; OAA, oxaloacetate; PDH, pyruvate dehydrogenase. 


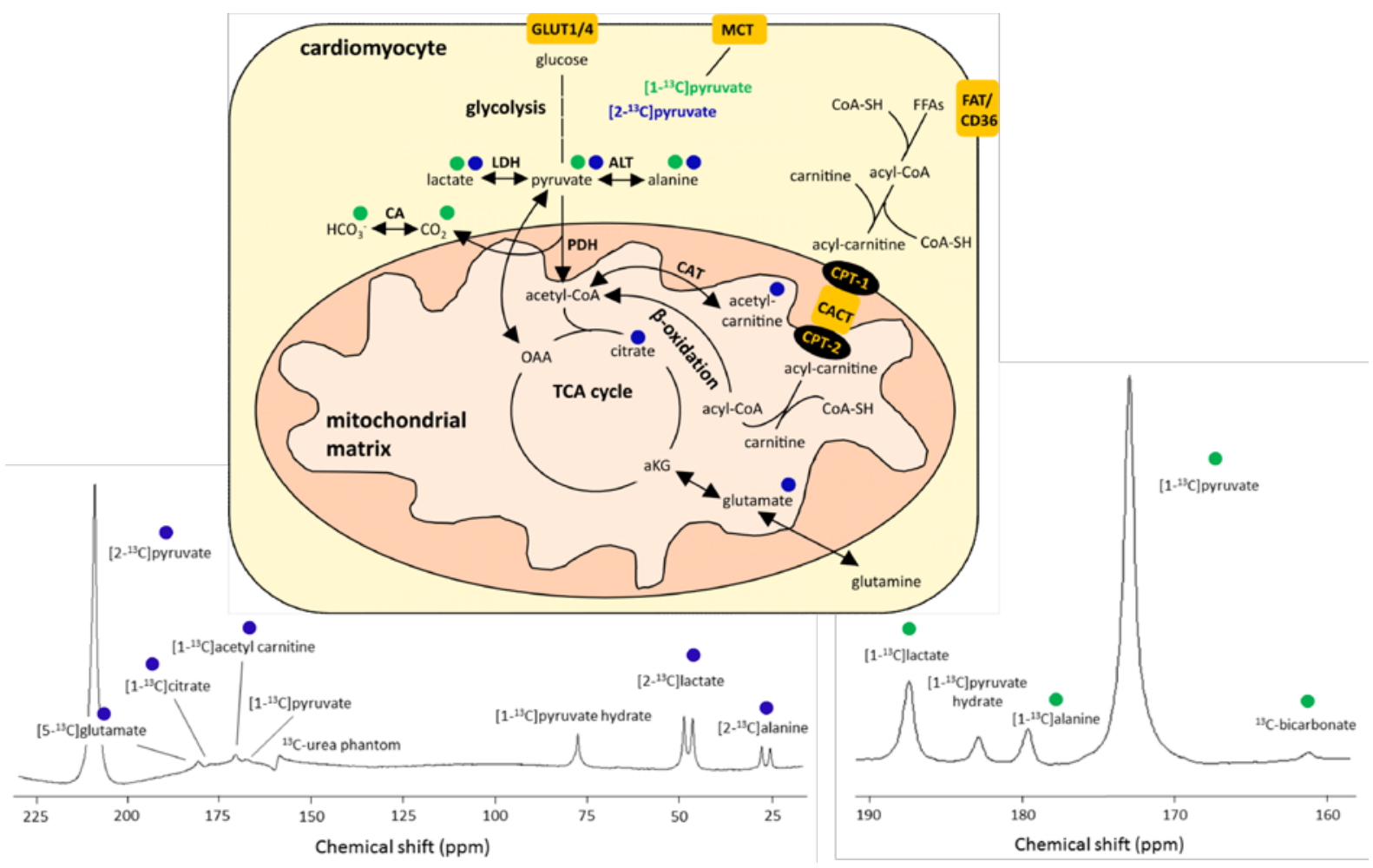

Figure 2 Measuring TCA cycle flux with hyperpolarised $\left[1-{ }^{13} \mathrm{C}\right]-$ and $\left[2-{ }^{13} \mathrm{C}\right]$ pyruvate

Cartoon showing the metabolic fate of hyperpolarised $\left[1-{ }^{13} \mathrm{C}\right]-$ and $\left[2-{ }^{13} \mathrm{C}\right]$ pyruvate in the cardiomyocyte (top) and MR spectra of a representative hyperpolarised $\left[2-{ }^{13} \mathrm{C}\right]$ pyruvate (bottom left) and $\left[1-{ }^{13} \mathrm{C}\right]$ pyruvate (bottom right) scan in rat hearts. Hyperpolarised $\left[1-{ }^{13} \mathrm{C}\right]-$ and $\left[2-{ }^{13} \mathrm{C}\right]$ pyruvate enter the cardiomyocyte through monocarboxylate transporters (MCT). The ${ }^{13} \mathrm{C}$ label in [1${ }^{13} \mathrm{C}$ ]pyruvate can be exchanged into the existing lactate pool through lactate dehydrogenase (LDH), or alternatively $\left[1-{ }^{13} \mathrm{C}\right]$ pyruvate can be oxidatively decarboxylated to acetyl-CoA yielding carbon dioxide $\left(\mathrm{CO}_{2}\right)$ in the reaction catalysed by pyruvate dehydrogenase $(\mathrm{PDH})$. The $\mathrm{CO}_{2}$ is in rapid exchange with tissue bicarbonate $\left(\mathrm{HCO}_{3}{ }^{-}\right)$through carbonic anhydrase (CA). $\left[1-{ }^{13} \mathrm{C}\right]$ pyruvate can also be transaminated to alanine in the reaction catalysed by alanine aminotransferase (ALT). [2${ }^{13} \mathrm{C}$ ]pyruvate can also yield ${ }^{13} \mathrm{C}$-labelled lactate and alanine. However, the ${ }^{13} \mathrm{C}$-label is retained in acetyl-CoA after oxidative decarboxylation by PDH and enters the tricarboxylic acid (TCA) cycle. Acetyl-CoA binds with oxaloacetate (OAA) in the reaction catalysed by citrate synthase to form citrate. The ${ }^{13} \mathrm{C}$-label in citrate can be transferred into the glutamate pool via several enzymatic steps. The ${ }^{13} \mathrm{C}$-label in acetyl-CoA can furthermore end up in the acetyl-carnitine pool through synthesis of carnitine and acetyl-CoA, catalysed by reversible carnitine-acetyltransferase (CAT). Green and blue dots represent metabolites that can be detected by MR spectroscopy in the heart following injection of hyperpolarised $\left[1-{ }^{13} \mathrm{C}\right]-$ and $\left[2-{ }^{13} \mathrm{C}\right]$ pyruvate, respectively. $\left[1-{ }^{13} \mathrm{C}\right]$ pyruvate hydrate is a nonmetabolically active contaminant arising through the (reversible) heat-induced hydration of hyperpolarised $\left[1-{ }^{13} \mathrm{C}\right]$ pyruvate during the dissolution. The peak labelled ${ }^{13} \mathrm{C}$-urea phantom in the left spectrum is from an $8 \mathrm{M}{ }^{13} \mathrm{C}$-urea-containing phantom attached to the carbon-13 coil next to the rat to allow for pulse calibration. Other abbreviations used in this figure: aKG, alpha-ketoglutarate; CACT, carnitine-acylcarnitine translocase; CPT, carnitine palmitoyltransferase; FAT, fatty acid transporter; FFAs, fatty acids; GLUT, glucose transporter. 


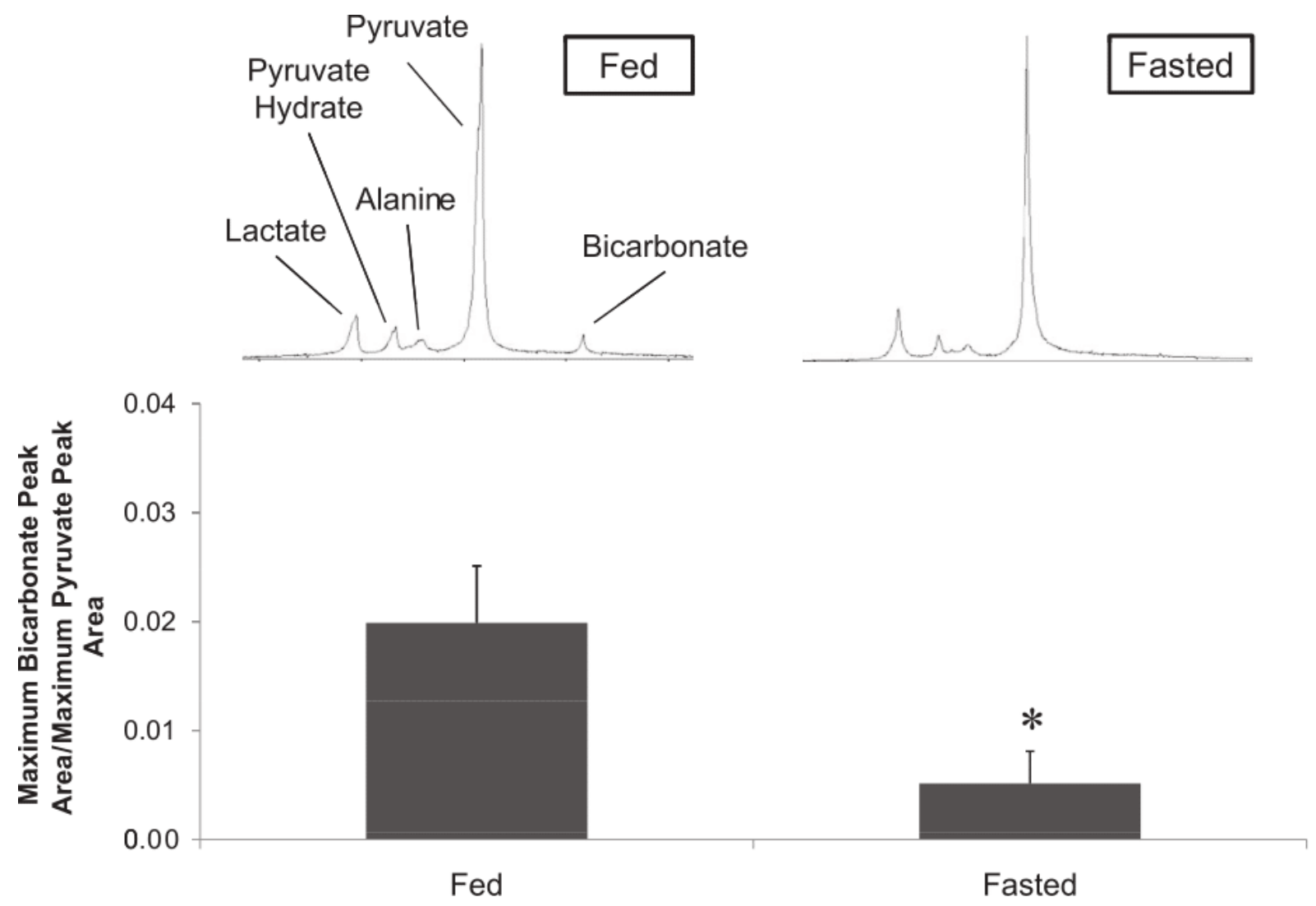

Figure 3 Hyperpolarised $\left[1-{ }^{13} \mathrm{C}\right]$ pyruvate MR spectroscopy in healthy rat hearts

Bicarbonate:pyruvate ratio in fed male Wistar rats compared with overnight fasted rats $(\mathrm{n}=6$; *, $\mathrm{p}<0.001)$. Representative single spectra, acquired at $\mathrm{t}=10 \mathrm{~s}$ after hyperpolarised $\left[1-{ }^{13} \mathrm{C}\right]$ pyruvate injection into the tail vein, illustrate the difference in bicarbonate production. Copyright (2008) National Academy of Sciences, figure taken and legend adapted with permission from [292]. 


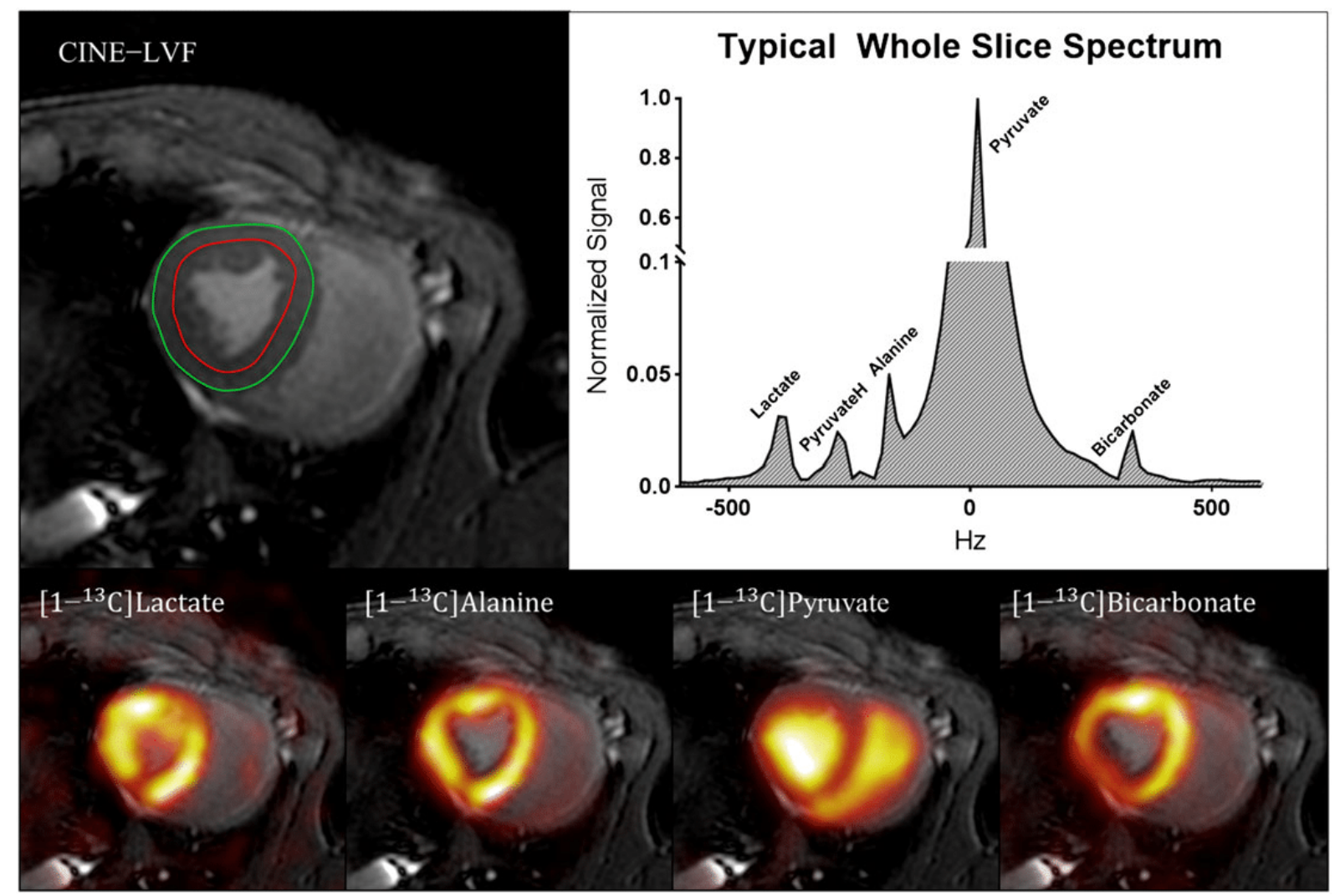

Figure 4 Hyperpolarised $\left[1-{ }^{13} \mathrm{C}\right]$ pyruvate imaging in a healthy pig heart after GIK infusion

Top left: CINE left ventricular function (CINE-LVF) showing the mid-ventricular short axis of a healthy female Danish domestic pig heart with a region of interest (ROI) drawn to outline the endocardium and epicardium for the hyperpolarised images. Top right: a representative spectrum 120 min after glucose-insulin-potassium (GIK) infusion with the signal from a $50 \mathrm{~mm}$ image slice. Bottom: lactate, alanine, pyruvate and bicarbonate hyperpolarised images are overlaid on the CINELVF image. Figure taken and legend adapted from [107] with permission of John Wiley and Sons, Inc. 

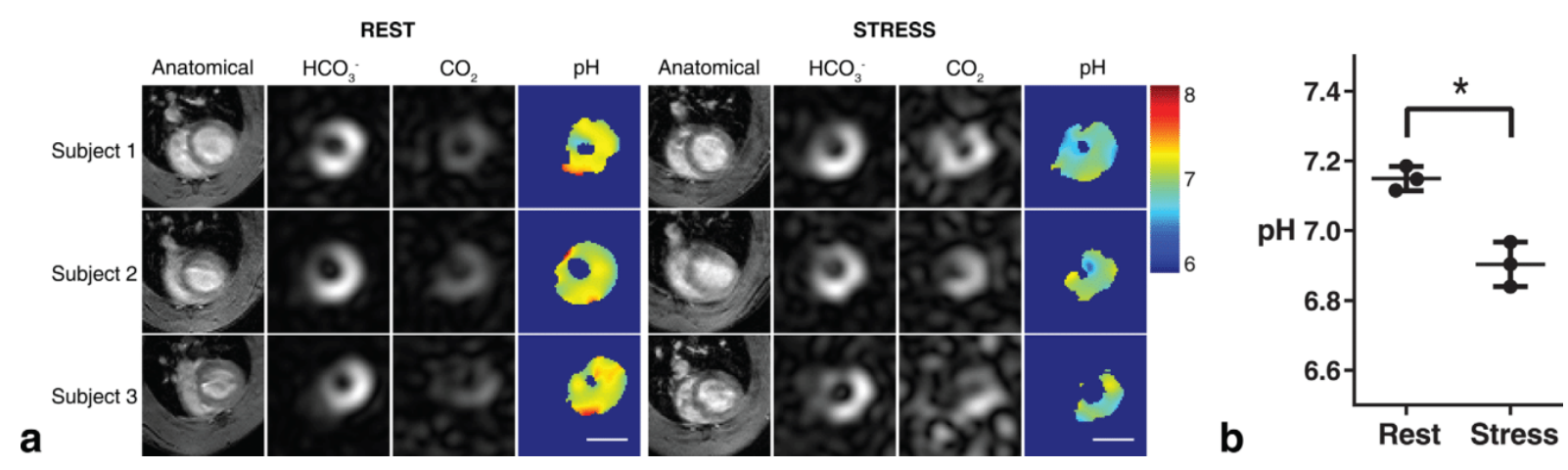

Figure 5 Intracellular $\mathrm{pH}$ imaging with hyperpolarised $\left[1-{ }^{13} \mathrm{C}\right]$ pyruvate in rat hearts at rest and under dobutamine stress

a) Intracellular $\mathrm{pH}$ measurements obtained using the ratio between $\mathrm{HCO}_{3}{ }^{-}$and $\mathrm{CO}_{2}$ from a midventricular slice of a male Wistar rat heart. Images are cropped to a 30x80 $\mathrm{mm}^{2} \mathrm{FOV}$. The scale bar indicates $1 \mathrm{~cm}$. The ${ }^{13} \mathrm{C}$ images are taken from a single time point, 20 seconds after the start of injection of hyperpolarised $\left[1-{ }^{13} \mathrm{C}\right]$ pyruvate. b) Increased cardiac workload through dobutamine infusion results in decreased apparent $\mathrm{pH}_{\mathrm{i}}$ in the healthy heart. ${ }^{*}=\mathrm{p}<0.05$. Figure taken and legend adapted from [138] with permission of John Wiley and Sons, Inc. 
a. Sham

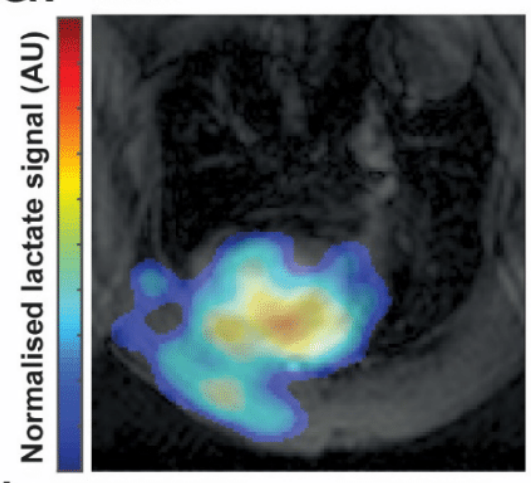

b.

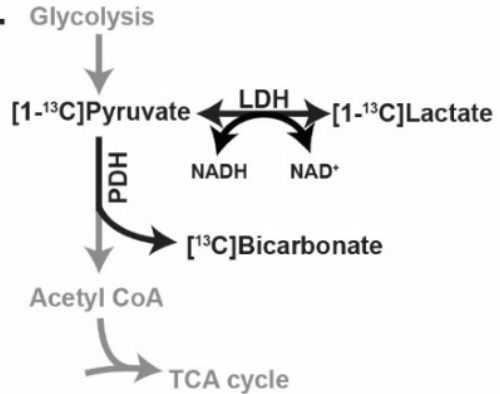

Infarct

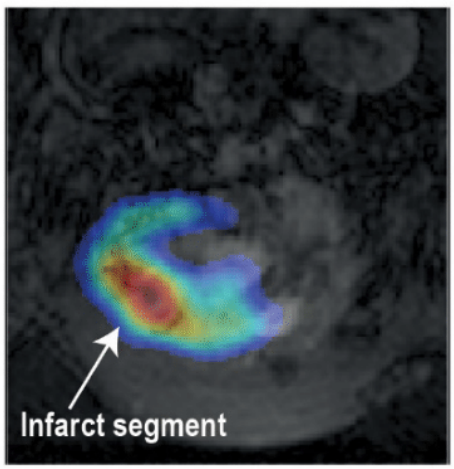

Cardiac $\left[{ }^{13} \mathrm{C}\right]$ lactate signal

Day 3

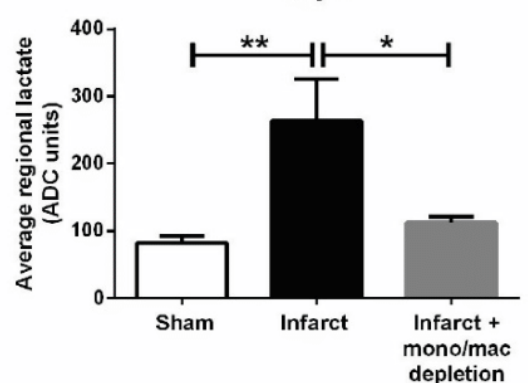

Infarct +

mono/mac depletion

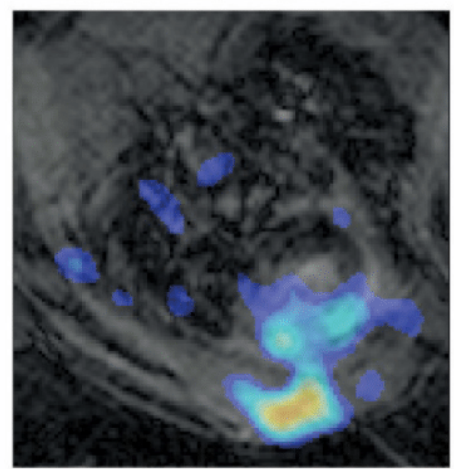

Cardiac $\left[{ }^{13} \mathrm{C}\right]$ lactate signal

Day 7

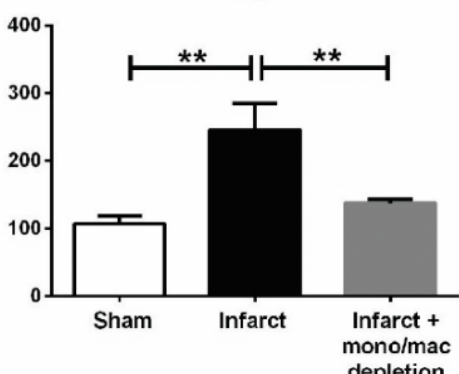

Figure 6 Imaging hyperpolarised $\left[1-{ }^{13} \mathrm{C}\right]$ pyruvate metabolism in a rat model of myocardial infarction

Panel a) and b): MRI following injection of hyperpolarised $\left[1-{ }^{13} \mathrm{C}\right]$ pyruvate demonstrates intense [1$\left.{ }^{13} \mathrm{C}\right]$ lactate signal at both day 3 and day 7 post experimental myocardial infarction in female Wistar rat hearts. The $\left[1-{ }^{13} \mathrm{C}\right]$ lactate signal was normalised in rats undergoing pharmacological macrophage depletion. Macrophage depletion was performed in $\mathrm{n}=6$ rats assigned per timepoint per group (total $\mathrm{n}=36$ ) to give 4-6 evaluable datasets per timepoint per group, mean $\pm S E M$, statistical comparison by one-way ANOVA with the Holm-Sidak correction for multiple comparisons. ${ }^{*} \mathrm{p}<0.05,{ }^{* *} \mathrm{p}<0.01$. Adapted from [159] with permission of Wolters Kluwer Health, Inc. 


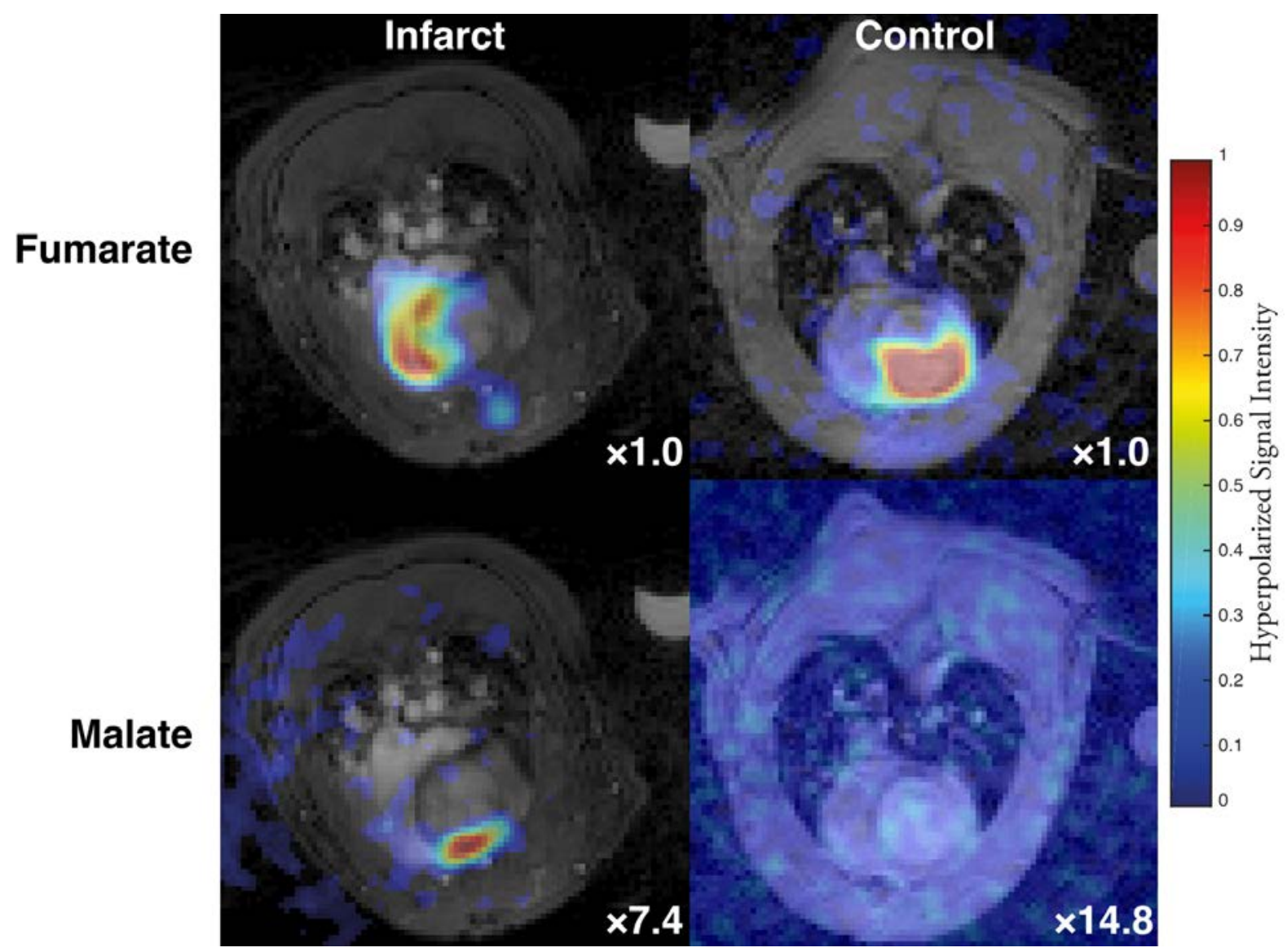

Figure 7 Imaging tissue necrosis with hyperpolarised $\left[1,4-{ }^{13} \mathrm{C}_{2}\right]$ fumarate in a rat model of myocardial infarction

$\left[1,4-{ }^{13} \mathrm{C}_{2}\right]$ Malate production was only visible in hearts of infarcted female Wistar rats, and was localised to the anterior region of the chest wall, consistent with the location of the insult. The colour axis is linear for the 4 hyperpolarised datasets, with the maximum level scaled down by the factor shown. Copyright (2017) Journal of the American College of Cardiology: Cardiovascular Imaging, licensed CC-BY and adapted with permission from [142]. 

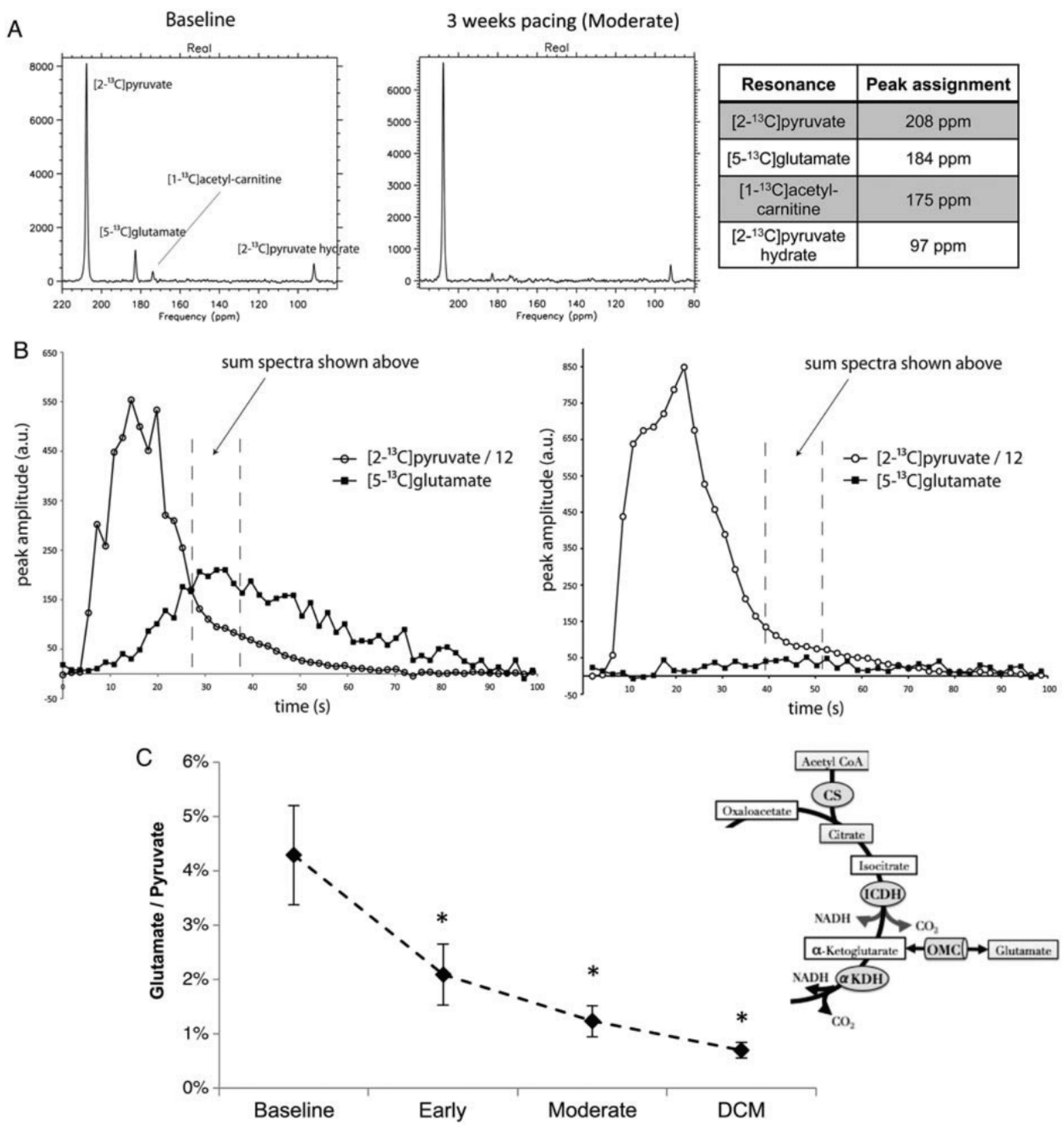

Figure 8 Hyperpolarised $\left[2-{ }^{13}\right.$ C]pyruvate MR spectroscopy in a pig model of dilated cardiomyopathy

Hyperpolarised ${ }^{13} \mathrm{C}$ magnetic resonance spectroscopy (MRS) showing altered $\left[5-{ }^{13} \mathrm{C}\right]$ glutamate production during development of dilated cardiomyopathy (DCM), following infusion of hyperpolarised $\left[2-{ }^{13} \mathrm{C}\right]$ pyruvate. (A) Representative spectra taken from a healthy pig (left), and from the same pig after 3 weeks of pacemaker-induced right-ventricular pacing, when it had moderate cardiac dysfunction. (B) Representative time course of the infused $\left[2-{ }^{13} \mathrm{C}\right]$ pyruvate and its conversion into $\left[5-{ }^{13} \mathrm{C}\right]$ glutamate, following quantification of the spectra shown in A. (C) The [5$\left.{ }^{13} \mathrm{C}\right]$ glutamate/[2- $\left.{ }^{13} \mathrm{C}\right]$ pyruvate ratio, measured for all pigs during the development of DCM. ${ }^{*} \mathrm{p}<0.05$. Taken from [293] with permission of John Wiley and Sons, Inc. 


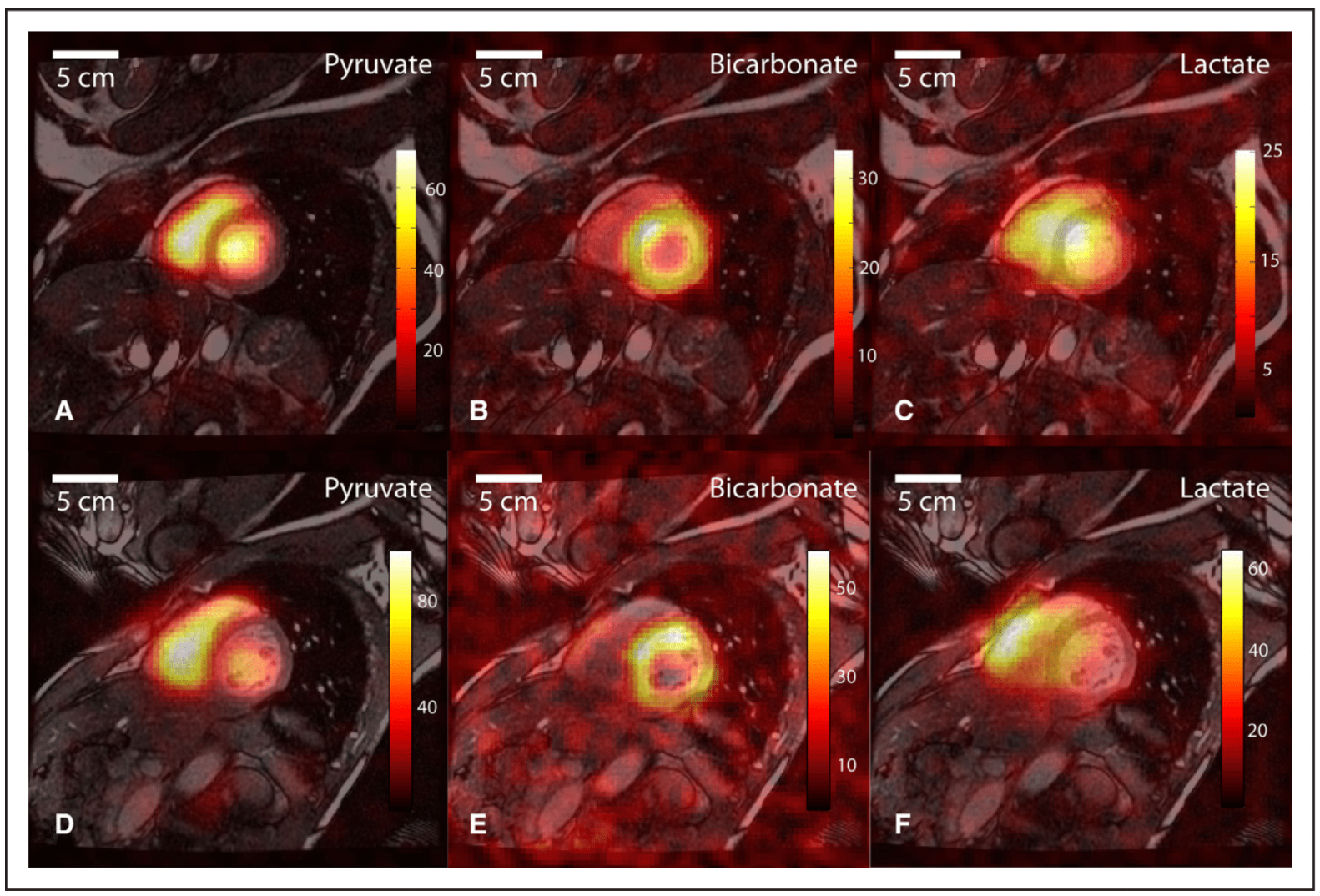

Figure 9 First in-man hyperpolarised $\left[1{ }^{13} \mathrm{C}\right]$ pyruvate MR spectroscopy of the heart

Representative ${ }^{13} \mathrm{C}$ image displayed as colour overlays on top of grayscale anatomic images in a midleft ventricle LV) slice from two different subjects (A-C and D-F). The $\left[1-{ }^{13} \mathrm{C}\right]$ pyruvate substrate was seen mainly in the blood pool within the cardiac chambers (A and D). Flux of pyruvate through the pyruvate dehydrogenase complex is reflected in the ${ }^{13} \mathrm{C}$-bicarbonate images (B and E), with signal predominantly in the wall of the LV. The $\left[1-{ }^{13} \mathrm{C}\right]$ lactate signal $(\mathrm{C}$ and $\mathrm{F})$ appears with a diffuse distribution covering the muscle and chambers. Taken from [164] with permission of Wolters Kluwer Health, Inc. 